\title{
Forward dijets in proton-nucleus collisions at next-to-leading order: the real corrections
}

\author{
Edmond Iancu ${ }^{a}$ and Yair Mulian ${ }^{b, c}$ \\ ${ }^{a}$ Institut de Physique Théorique, Universite Paris Saclay, CNRS, CEA, \\ F-91191, Gif-sur-Yvette, France \\ ${ }^{b}$ Department of Physics, University of Jyväskylä, \\ P.O. Box 35, 40014 University of Jyväskylä, Finland \\ ${ }^{c}$ Helsinki Institute of Physics, \\ P.O. Box 64, 00014 University of Helsinki, Finland
}

E-mail: edmond.iancu@ipht.fr, yair25m@gmail.com

ABSTRACT: Using the CGC effective theory together with the hybrid factorisation, we study forward dijet production in proton-nucleus collisions beyond leading order. In this paper, we compute the "real" next-to-leading order (NLO) corrections, i.e. the radiative corrections associated with a three-parton final state, out of which only two are being measured. To that aim, we start by revisiting our previous results for the three-parton cross-section presented in [1]. After some reshuffling of terms, we deduce new expressions for these results, which not only look considerably simpler, but are also physically more transparent. We also correct several errors in this process. The real NLO corrections to inclusive dijet production are then obtained by integrating out the kinematics of any of the three final partons. We explicitly work out the interesting limits where the unmeasured parton is either a soft gluon, or the product of a collinear splitting. We find the expected results in both limits: the B-JIMWLK evolution of the leading-order dijet cross-section in the first case (soft gluon) and, respectively, the DGLAP evolution of the initial and final states in the second case (collinear splitting). The "virtual" NLO corrections to dijet production will be presented in a subsequent publication.

KeYwords: Heavy Ion Phenomenology, NLO Computations

ArXiv EPrint: 2009.11930 


\section{Contents}

1 Introduction 1

2 Dijet cross-section at leading-order 4

2.1 Kinematics 5

2.2 The quark-gluon component of the quark light-cone wavefunction 6

$\begin{array}{lll}2.3 & \text { The quark-gluon cross-section at leading order } & 7\end{array}$

3 Trijet cross-section at leading order $\quad 11$

$\begin{array}{ll}3.1 \text { The tri-parton components of the quark outgoing state } & 12\end{array}$

$\begin{array}{lll}3.1 .1 & \text { The tri-quark final state } & 12\end{array}$

$\begin{array}{ll}\text { 3.1.2 The final state with one quark and two gluons } & 17\end{array}$

$\begin{array}{ll}3.2 & \text { The trijet cross-section } \\ & 20\end{array}$

$\begin{array}{lll}3.2 .1 & \text { The } q q \bar{q} \text { final state } & 20\end{array}$

$\begin{array}{ll}3.2 .2 & \text { The } q g g \text { final state } \\ \end{array}$

4 Next-to-leading order corrections: the real terms $\quad 29$

$\begin{array}{ll}4.1 \text { The di-quark jet production } & 29\end{array}$

4.2 The quark-antiquark di-jets 31

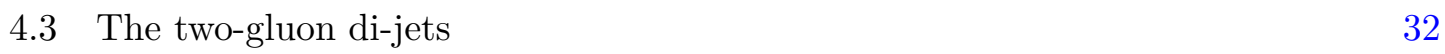

4.4 The quark-gluon dijets 33

5 Soft gluon emissions: recovering the B-JIMWLK evolution 33

5.1 Direct emissions by the quark 34

$\begin{array}{lll}5.2 & \text { Direct contributions involving the 3-gluon vertex } & 39\end{array}$

$\begin{array}{lll}5.3 & \text { Interference graphs } & 41\end{array}$

6 Collinear divergences: recovering the DGLAP evolution 45

$\begin{array}{lll}\text { 6.1 DGLAP evolution for the initial quark } & 46\end{array}$

$\begin{array}{ll}\text { 6.2 DGLAP evolution for the final quark } & 51\end{array}$

6.3 DGLAP evolution for the final gluon: $g \rightarrow g g$ splitting $\quad 54$

6.4 DGLAP evolution for the final gluon: $g \rightarrow q \bar{q}$ splitting 56

$\begin{array}{ll}\text { A Definitions of states and field quantization } & 57\end{array}$

$\begin{array}{ll}\text { B Matrix elements } & 59\end{array}$ 


\section{Introduction}

In this paper we shall study inclusive dijet production in proton-nucleus $(p A)$ collisions at forward rapidities, i.e. at very small angles with respect to the collision axis in the fragmentation region of the proton. This kinematics is interesting in that it gives us access to the small- $x$ part of the gluon distribution in the nuclear target, where high-density phenomena like gluon saturation are expected to be important. For this particular setup, it is a good approximation to assume that the final state - the two measured jets possibly accompanied by unmeasured particles - is produced via radiation from a single parton from the incoming proton. The role of the scattering is to put on-shell the otherwise virtual quanta from the parton (light-cone) wavefunction and also to give these quanta a non-trivial distribution in energies and momenta. In particular, their final distribution in transverse momenta (or azimuthal angles) should reflect properties of the nuclear gluon distribution, that we are ultimately interested in. To avoid a proliferation of cases, we shall restrict ourselves to the quark channel, that is, we shall consider only the case where the parton from the proton which participates in the scattering is a quark. The addition of the gluon channel is in principle straightforward and will be addressed in a subsequent publication.

As implicit in the previous discussion, we shall use the framework of the so-called "hybrid factorisation" [2-4], where the leading quark is assumed to be collinear with the proton (and described by the standard quark distribution), whereas its interactions with the nuclear target are described within the Colour Glass Condensate (CGC) effective theory [5, 6]. The nucleus is viewed as a source of strong, random, colour fields representing the small- $x$ gluons and their correlations. The high-energy scattering between a parton from the proton - the leading quark and its radiation - and these strong colour fields will be computed in the eikonal approximation, that is, by associating a Wilson line (describing colour precession) to each parton projectile. As a result, the cross-section corresponding to a given (partonic) final state can be related to gauge-invariant products of Wilson lines, whose high-energy evolution (via soft gluon emissions) is described by the BalitskyJIMWLK equations [7-14] - actually, an infinite hierarchy of coupled equations for multipoint correlations. This evolution becomes considerably simpler in the limit of a large number $N_{c} \gg 1$ of colours, where the hierarchy acquires a "triangular" structure. In particular, the first equation in this hierarchy reduces to a closed, non-linear, equation the Balitsky-Kovchegov equation [7, 8] — for the elastic $S$-matrix of a colour dipole.

The formalism as a whole - meaning the non-linear evolution equations with increasing energy and the hybrid factorisation - has been originally proposed to leading order (LO) in perturbative QCD [4-6]. But for realistic applications to the phenomenology, one needs next-to-leading order (NLO) accuracy, at least. Note that, unlike for the collinear factorisation (and the associated DGLAP evolution), the compatibility between the high-energy factorisation and the weak coupling expansion is a a priori unclear, at both conceptual and practical level. Yet, via explicit calculations and tenuous efforts, one was able to build the NLO versions of the BK [15] and B-JIMWLK [15-19] equations, and also of the "impact factors" (the scattering matrix elements void of evolution) for a few, 
relatively simple, processes, like single inclusive hadron production in $p A$ collisions [20$22]$, the structure functions for electron-proton $(e p)$ or electron-nucleus $(e A)$ deep inelastic scattering (DIS) at small Bjorken $x$ [23-25], exclusive diffractive vector-meson and dijet production in DIS at small $x$ [26-28], and inclusive photon + dijet production in $e A$ collisions at small $x$ [29].

The original NLO results for both the BK equation and (some of) the impact factors turned out to be problematic, in the sense of generating instabilities in the high energy evolution [30], or negative cross-sections for particle production [31-34]. These difficulties have been eventually understood and cured. In the case of the BK equation, the solution [35-42] involves all-order resummations which enforce the proper time-ordering for the lifetimes of the soft gluon fluctuations of the dilute projectile (a parton from the proton, or a colour dipole in the case of DIS). The scheme-dependence of such resummations is considerably reduced when using the rapidity of the dense target (the large nucleus) as the "evolution time" [40]. For the NLO impact factors, the negativity problem arises when enforcing a separation between the leading-order BK evolution and the NLO impact factor which is local in rapidity [43] (thus following the traditional prescription of the $k_{T}$-factorisation $[44,45]$ ). So, the simplest way to avoid the problem - and obtain a positive-definite cross-section - is to give up this separation, that is, to keep the high-energy evolution and the NLO corrections together, in a generalised impact factor [43, 46-48]. In this "unsubtracted" scheme, the only evolution which needs to be factorised from the "hard" impact factor is the DGLAP evolution on the external lines - that is, the evolution of the parton distribution for the parton from the proton which initiates the scattering, and that of the fragmentation functions for the produced hadrons.

One process that has attracted much interest over the last years is the production of a pair of jets or hadrons in "dilute-dense" — proton(deuteron)-nucleus $(p A)$ [49-58] or electron-nucleus $(e A)[51,59-65]$ - collisions at forward rapidities. This process could be used to probe saturation even in the (experimentally more accessible) set-up where the final jets/hadrons have transverse momenta significantly larger than the saturation momentum in the dense target. Indeed, the multiple scattering off the saturated gluons in the target is one of the mechanisms responsible for the transverse momentum imbalance between the final jets, hence for their distribution in the relative azimuthal angle. (The other important such a mechanism is the final state radiation, leading to the so-called "Sudakov factor" [66].) In turn, this imbalance leads to a broadening of the final particles distribution in the relative azimuthal angle $\Delta \phi$, around the back-to-back peak at $\Delta \phi=\pi$. Such a broadening has indeed been observed in $\mathrm{d}+\mathrm{Au}$ collisions at RHIC [67, 68], although at this level it looks difficult to distinguish the effects of saturation from those of the final state radiation [69].

That said, it would be important to have a more precise calculation of the cross-section for dijets (or dihadrons) production within the CGC formalism. This is our main purpose in this and a subsequent paper, where we will compute the respective NLO corrections to the impact factor for the case of $p A$ collisions. Specifically, in this paper we shall present the "real" NLO corrections - those associated with a final state which involves three partons, out of which only two are measured. By integrating out the kinematics of the un- 
measured parton, one generates a NLO correction to the cross-section for dijet production. In the companion paper, we will compute the corresponding "virtual" corrections - those associated with one-loop corrections to the amplitude.

For simplicity and to avoid a proliferation of cases, we shall restrict ourselves to the quark channel, that is, we shall only consider processes in which the final state is obtained via radiation from an initial quark from the wavefunction of the incoming proton. The quark channel is expected to dominate the forward particle production in the kinematics at RHIC, but the gluon channel should be added too in view of realistic applications to the LHC. We plan to do so in a later paper.

The starting point for computing the "real" NLO corrections to the dijet cross-section is the leading-order (tree-level) cross-section for "trijet" (three partons) production, that we obtained in a previous paper [1], by using the formalism of the light-cone wavefunction (LCWF). So, for our present purposes, it should be enough to "integrate out" one (any) of the three final partons in our results in [1]. However, this is not what we shall do in practice. Indeed, the LO trijet cross-section is by itself a very complex quantity and our respective results in [1] were presented in a cumbersome way, which is not convenient for the present purposes. (We have realised that when trying to match our results for "real" and "virtual" corrections to dijet production, e.g. in order to check the cancellation of the infrared divergences.) So, our first step in this paper will be to re-derive the results for 3 -parton production in a streamlined way and with a better strategy for organising the final expressions. In this process, we shall also correct several errors which occurred in our original results [1], that we were able to identify thanks to various tests to be discussed below.

In view of the above, the results for the LO trijet cross-section to be presented in this paper should be viewed as our final respective results, in replacement of the previous ones in [1]. These results are still formal, as they involve undone Fourier transforms in the transverse plane: the calculation of the LCWF is performed using the transverse coordinate representation, to take profit of the eikonal approximation. Hence, our expressions for the cross-section involve Fourier transforms relating the transverse coordinates of the three final partons (in both the amplitude and the complex conjugate amplitude) to their respective momenta, as measured in the final state. To actually compute these Fourier transforms say, in view of applications to the phenomenology - one would also need explicit results for the partonic $S$-matrices probed by these particular processes. The techniques for computing such $S$-matrices - from numerical solutions to the BK and JIMWLK equations, or via mean field approximations to the latter - are well documented in the literature, but their discussion goes beyond our present purposes. In agreement with previous studies [51, 70], we shall find that in the multicolour limit $N_{c} \rightarrow \infty$, the various $S$-matrices that we shall encounter are built with just two non-trivial colour structures: the dipole and the quadrupole.

Given these results for trijet production, the "real" NLO corrections to dijets are easily obtained (at least, formally) by integrating over the longitudinal and the transverse momentum of one of the three final partons and then convoluting with the proton distribution function for the incoming quark. If one measures two hadrons in the final state, convolutions with the appropriate parton-to-hadron fragmentation functions are also needed. 
As a check of our calculations, we shall study two special limits for which the results are a priori known: the case where the unmeasured parton is a soft gluon and that where this parton is produced via a collinear splitting. In the first case, we expect to recover (one step in) the B-JIMWLK evolution ${ }^{1}$ of the LO cross-section for producing a quarkgluon pair. In the second case, one should find the DGLAP evolution for the incoming quark distribution and for the fragmentation functions of the produced quark and gluon. We indeed recover these expected results, but only after rather non-trivial manipulations, which in particular involve cancellations between many terms. Thus the good results that we find in these limits provides a rather stringent test on our results.

We shall not insist in separating out the LO evolution from the NLO impact factor. In the case of the B-JIMWLK evolution, this is in line with our preference for the "unsubtracted" scheme [43] alluded to above, which avoids potential problems associated with a local subtraction in rapidity. In the case of the DGLAP evolution, the subtraction of the collinear divergences is indeed mandatory, but it requires more refined techniques like the dimensional regularisation, that we shall develop in the companion paper devoted to "virtual" NLO corrections.

This paper is structured as follows. In section 2 we shall briefly recall the result for the LO dijet cross-section in the quark channel, i.e. the Born-level cross-section for producing a quark-gluon ( $q g)$ pair at forward rapidities. This result is well known [1, 49, 51] but we shall often need it for comparisons with the NLO results to be obtained later. In section 3, we present the LO (tree-level) results for the production of three partons: $q q \bar{q}$ and $q g g$. We first show the three-parton components of the quark LCWF in the final state and then use them to compute the relevant cross-sections. As already mentioned, in doing that we shall both reorganise and correct our original results in [1]. In section 4 we compute the "real" NLO corrections to dijet production in the quark-initiated channel by (formally) "integrating out" one of the three partons in the trijet results. We also explain the simplifications which occur in the colour structure (i.e. in the partonic $S$-matrices) due to the fact that one of the partons is not measured. In section 5 we show that in the limit where the unmeasured parton is a soft gluon, our NLO results reproduce, as expected, the (real part of the) JIMWLK evolution for the $q g$ dijet cross-section. Finally, in section 6, we consider the limit where the unmeasured parton is produced by a collinear splitting. We show that, in this limit, our NLO results develop collinear singularities, that we isolate to leading logarithmic accuracy and verify that they can be interpreted as one-step in the DGLAP evolution of the initial $(q)$ and final $(q g)$ states.

\section{Dijet cross-section at leading-order}

As a warm-up, let us briefly recall some steps in the derivation of the leading-order (LO) result for the cross-section for dijet production in $p A$ collisions at forward rapidity (see e.g. $[1,49]$ for more details). As mentioned in the Introduction, we consider only the quark

\footnotetext{
${ }^{1}$ More precisely, one must recover only the "real" part of the B-JIMWLK equation and similarly for the DGLAP equation; the respective "virtual" parts will be generated by the "virtual" NLO corrections to the dijet cross-section.
} 
channel: the parton collinear with the incoming proton and which initiates the process is taken to be a quark. (The corresponding result for the gluon channel can be found in refs. $[1,53]$.)

\subsection{Kinematics}

We start with some generalities on the kinematics and use this opportunity to introduce some of the notations. We chose the $z$ direction along the collision axis and work in a frame in which the proton is an energetic right-mover with (light-cone) longitudinal momentum $Q^{+}$, whereas the nucleus is an ultrarelativistic left mover, with longitudinal momentum $P^{-}$per nucleon. (We shall neglect the nucleon masses in what follows.) We more precisely assume that the nucleus target carries most of the total energy, so the high-energy evolution via the successive emissions of soft gluons is fully encoded in the nuclear gluon distribution.

To leading order, dijet production at forward rapidities and in the quark channel proceeds as follows (see also figure 1): a quark initially collinear with the proton, with longitudinal momentum $q^{+}=x_{q} Q^{+}$, scatters off the dense gluon system in the nuclear target and emits a gluon in the process. The two "jets" are the final quark, with longitudinal momentum $p^{+}=x_{1} Q^{+}$and transverse momentum $\boldsymbol{p}$, and the emitted gluon, with $k^{+}=$ $x_{2} Q^{+}$and final transverse momentum $\boldsymbol{k}$. The target has zero "plus" momentum, hence the respective component is preserved by the scattering: $x_{q}=x_{1}+x_{2}$; accordingly, we shall also write $k^{+}=\theta q^{+}$and hence $p^{+}=(1-\theta) q^{+}$, with $\theta=x_{2} / x_{q} \leq 1$ the gluon splitting fraction. On the other hand, the collision can transmit a transverse momentum of the order of the saturation momentum $Q_{s}$ - the typical momentum of a gluon at saturation -, hence we expect an imbalance $|\boldsymbol{p}+\boldsymbol{k}| \sim Q_{s}$ between the final jets.

The two jets are put on-shell by the collision, hence they must receive from the nucleus a "minus" component equal to their total light-cone energy; writing this as a fraction $x_{g}$ of $P^{-}$, we deduce

$$
x_{g} P^{-}=\frac{\boldsymbol{p}^{2}}{2 p^{+}}+\frac{\boldsymbol{k}^{2}}{2 k^{+}} \Longrightarrow x_{g}=\frac{\boldsymbol{p}^{2}}{x_{1} s}+\frac{\boldsymbol{k}^{2}}{x_{2} s},
$$

with $s=2 Q^{+} P^{-}$(the center-of-mass energy squared of the collision). It is customary to express the longitudinal fractions $x_{q}$ and $x_{g}$ in terms of the (pseudo)rapidities $\eta_{1} \equiv$ $(1 / 2) \ln \left(p^{+} / p^{-}\right)$and $\eta_{2} \equiv(1 / 2) \ln \left(k^{+} / k^{-}\right)$of the produced jets in the center-of-mass frame, where $Q^{+}=P^{-}=\sqrt{s / 2}$. Using $p^{+}=\left(p_{\perp} / \sqrt{2}\right) \mathrm{e}^{\eta_{1}}$ and similarly $k^{+}=\left(k_{\perp} / \sqrt{2}\right) \mathrm{e}^{\eta_{2}}$ (with $p_{\perp} \equiv|\boldsymbol{p}|$ etc), one finds

$$
x_{q}=\frac{p_{\perp}}{\sqrt{s}} \mathrm{e}^{\eta_{1}}+\frac{k_{\perp}}{\sqrt{s}} \mathrm{e}^{\eta_{2}}, \quad x_{g}=\frac{p_{\perp}}{\sqrt{s}} \mathrm{e}^{-\eta_{1}}+\frac{k_{\perp}}{\sqrt{s}} \mathrm{e}^{-\eta_{2}} .
$$

The forward dijet kinematics corresponds to the situation where $\eta_{1}$ and $\eta_{2}$ are both positive and larger than 1 . In this regime, one has $x_{g} \ll x_{q}<1$, showing that the forward particle production explores the small- $x_{g}$ part of the nuclear wavefunction. The relevant value of the nuclear saturation momentum increases with decreasing $x_{g}$, due to the rise in the gluon density in the transverse plane, via soft gluon emissions. This is governed by the non-linear 
evolution equations (BK or B-JIMWLK), which imply $Q_{s}^{2}\left(x_{g}\right) \sim x_{g}^{-\lambda_{s}}$, with $\lambda_{s} \simeq 0.20$ (see e.g. [41] for a recent study).

Note that in deriving eq. (2.2) we did not necessarily assume $2 \rightarrow 2$ kinematics (i.e. $q g \rightarrow q g$ ). In fact, the most interesting case for us here is that of multiple scattering, which involves the exchange of arbitrarily many soft gluons between the quark-gluon fluctuation of the proton and the nucleus. In such a case, $x_{g} P^{-}$is the total LC energy transmitted from the target to the quark-gluon system and $\boldsymbol{p}+\boldsymbol{k}$ is similarly the total transverse momentum.

Under the present assumptions, multiple scattering can be resummed to all orders within the eikonal approximation: the nuclear target appears to the proton as a Lorentzcontracted shockwave (say, localised at $x^{+}=0$ ) and the transverse coordinates of a projectile parton (quark or gluon) is not modified by the collision. It is then convenient to work in the transverse coordinate representation and Fourier transform to transverse momenta only at the end of the calculation. In this representation, the only effect of the scattering is a colour precession of the parton wavefunction, represented by a Wilson line in the appropriate representation of the colour group $\mathrm{SU}\left(N_{c}\right)$.

\subsection{The quark-gluon component of the quark light-cone wavefunction}

We use the light-cone wavefunction (LCWF) formalism and the projectile LC gauge $A^{+}=$ 0 . The initial state at (LC) time $x^{+} \rightarrow-\infty$ is taken to be a bare quark: $|q\rangle^{\text {in }}=\left|q_{\lambda}^{\alpha}\left(q^{+}, \boldsymbol{q}\right)\right\rangle$, with $q^{+}, \boldsymbol{q}, \lambda$ and $\alpha$ denoting the quark longitudinal and transverse momenta, its spin, and its colour state, respectively. (Our conventions for the bare Fock states and for the action of the associated creation and annihilation operators are presented in appendix A.) The outgoing state at $x^{+} \rightarrow \infty$ is computed as $|q\rangle^{\text {out }}=U_{I}(\infty, 0) \hat{S} U_{I}(0,-\infty)|q\rangle^{\text {in }}$, where the QCD evolution operators $U_{I}(0,-\infty)$ and $U_{I}(\infty, 0)$ describe QCD radiation prior and after the scattering, respectively, and $\hat{S}$ is the $S$-matrix operator for the scattering between the parton system which exists at time $x^{+}=0$ and the shockwave.

To compute quark-gluon production to leading order (LO), it is enough to expand the action of the evolution operators to $\mathcal{O}(g)$, e.g.

$$
U_{I}(0,-\infty)|i\rangle=|i\rangle-\sum_{j} \frac{\left\langle j\left|H_{\text {int }}\right| i\right\rangle}{E_{j}-E_{i}}|j\rangle+\cdots
$$

where $H_{\text {int }}$ is the interaction part of the Hamiltonian, $|i\rangle,|j\rangle$ are energy-momentum eigenstates of the free QCD Hamiltonian $H_{0}$ (i.e. the Fock states built with bare partons) and $E_{i}, E_{j}$ are the corresponding LC energies (the sum of the minus components of the partonic 4-momenta for all the partons composing a Fock state, assumed to be on-shell). Taking $|i\rangle=\left|q_{\lambda}^{\alpha}\left(q^{+}, \boldsymbol{q}\right)\right\rangle$, the outgoing state is a superposition of a single-quark state (whose colour has been rotated by the scattering) and the quark-gluon state, in which we are primarily interested. The $q g$ Fock-space component is most convenient written in the transverse coordinate representation, where the matrix elements of $\hat{S}$ are diagonal when computed in 
the eikonal approximation; it then reads [1]:

$$
\begin{aligned}
& \left|q_{\lambda}^{\alpha}\left(q^{+}, \boldsymbol{w}\right)\right\rangle_{q g}^{\text {out }}=-\int d^{2} \boldsymbol{x} d^{2} \boldsymbol{y} \int_{0}^{1} d \theta \frac{i g \phi_{\lambda_{1} \lambda}^{i j}(\theta) \sqrt{q^{+}}}{4 \sqrt{2 \theta} \pi^{2}} \frac{\boldsymbol{x}^{j}-\boldsymbol{y}^{j}}{(\boldsymbol{x}-\boldsymbol{y})^{2}} \delta^{(2)}(\boldsymbol{w}-(1-\theta) \boldsymbol{x}-\theta \boldsymbol{y}) \\
& \times\left[V^{\sigma \beta}(\boldsymbol{x}) U^{b a}(\boldsymbol{y}) t_{\beta \alpha}^{a}-t_{\sigma \beta}^{b} V^{\beta \alpha}(\boldsymbol{w})\right]\left|q_{\lambda_{1}}^{\sigma}\left((1-\theta) q^{+}, \boldsymbol{x}\right) g_{i}^{b}\left(\theta q^{+}, \boldsymbol{y}\right)\right\rangle,
\end{aligned}
$$

where $\boldsymbol{w}$ is the transverse coordinate of the incoming quark (conjugated to its transverse momentum $\boldsymbol{q}$ ), $\boldsymbol{x}$ and $\boldsymbol{y}$ are the transverse coordinates of the final quark and gluon, and the constraint $\boldsymbol{w}=(1-\theta) \boldsymbol{x}+\theta \boldsymbol{y}$ follows from the conservation of the transverse momentum. Furthermore, $g_{i}^{b}\left(\theta q^{+}, \boldsymbol{y}\right)$ is a gluon with transverse helicity $i$, colour state $b$, longitudinal momentum $\theta q^{+}$and transverse position $\boldsymbol{y}$. We also used the following notation for the spinor/helicity structure:

$$
\phi_{\lambda_{1} \lambda}^{i j}(\theta) \equiv \chi_{\lambda_{1}}^{\dagger}\left[(2-\theta) \delta^{i j}-i \theta \varepsilon^{i j} \sigma^{3}\right] \chi_{\lambda}=\delta_{\lambda \lambda_{1}}\left[(2-\theta) \delta^{i j}-2 i \theta \varepsilon^{i j} \lambda\right] .
$$

The two terms inside the square bracket in eq. (2.4) come from the action of the $S$-matrix $\hat{S}$ and refer to the situation when the scattering with the shockwave occurs before and respectively after the gluon emission (see figure 1). $U_{b a}(\boldsymbol{x})$ and $V_{\beta \alpha}(\boldsymbol{x})$ are Wilson lines in the adjoint (for the gluon) and respectively fundamental (for the quark) representation, which describe scattering in the eikonal approximation - i.e. under the assumption that the transverse coordinate of the projectile parton is not modified by its scattering off the gluon distribution in the target. In matrix notations,

$$
U(\boldsymbol{x})=\mathrm{T} \exp \left\{i g \int d x^{+} T^{a} A_{a}^{-}\left(x^{+}, \boldsymbol{x}\right)\right\}, \quad V(\boldsymbol{x})=\mathrm{T} \exp \left\{i g \int d x^{+} t^{a} A_{a}^{-}\left(x^{+}, \boldsymbol{x}\right)\right\} .
$$

with the colour field $A_{a}^{-}$representing the small- $x$ gluons in the target. In the CGC effective theory, this field is random and must be averaged out at the level of the cross-section.

The outgoing state in eq. (2.4) is clearly vanishing in the absence of scattering, i.e. in the limit where $U \rightarrow 1$ and $V \rightarrow 1$. Indeed, in the absence of any interaction (like the scattering off a nuclear target), the quark-gluon pair cannot be produced in the final state (since an on-shell quark cannot emit a gluon). Furthermore, using the identity

$$
\left[V^{\dagger}(\boldsymbol{y}) t^{b} V(\boldsymbol{y})\right]_{\beta \alpha}=U^{b a}(\boldsymbol{y}) t_{\beta \alpha}^{a},
$$

one sees that the difference of Wilson lines in eq. (2.4) also vanishes when $\boldsymbol{y} \rightarrow \boldsymbol{x}$. This is the expression of colour conservation: in the limit where the transverse separation between the daughter partons shrinks to zero, the quark-gluon pair scatters off the nuclear target in the same way as its parent quark, hence the bare quark and its $q g$ fluctuation cannot be disentangled by the collision.

\subsection{The quark-gluon cross-section at leading order}

The inclusive cross section for forward quark-gluon production in quark-nucleus $(q A)$ scattering can be computed as the average of the respective product of number density operators 


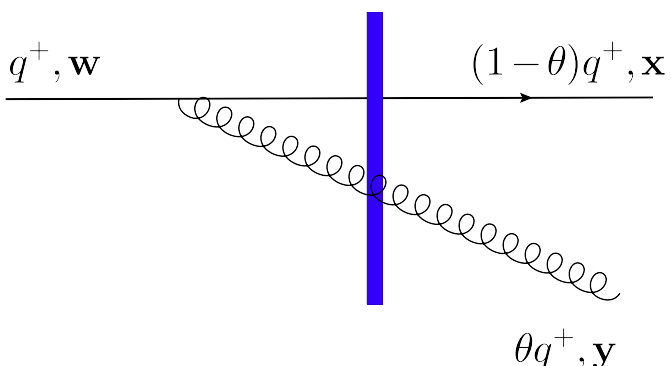

(a)

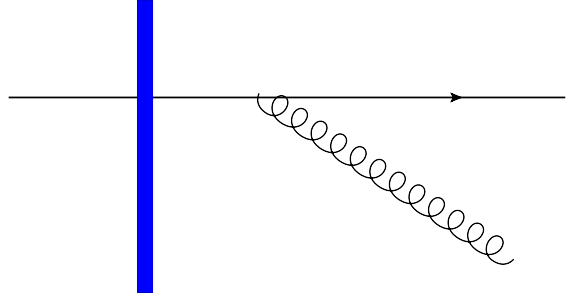

(b)

Figure 1. The quark-gluon component of the outgoing wave-function for an incoming quark. There are two ways to insert the shockwave, before and after the gluons emission, represented in figure a and $\mathrm{b}$, respectively.

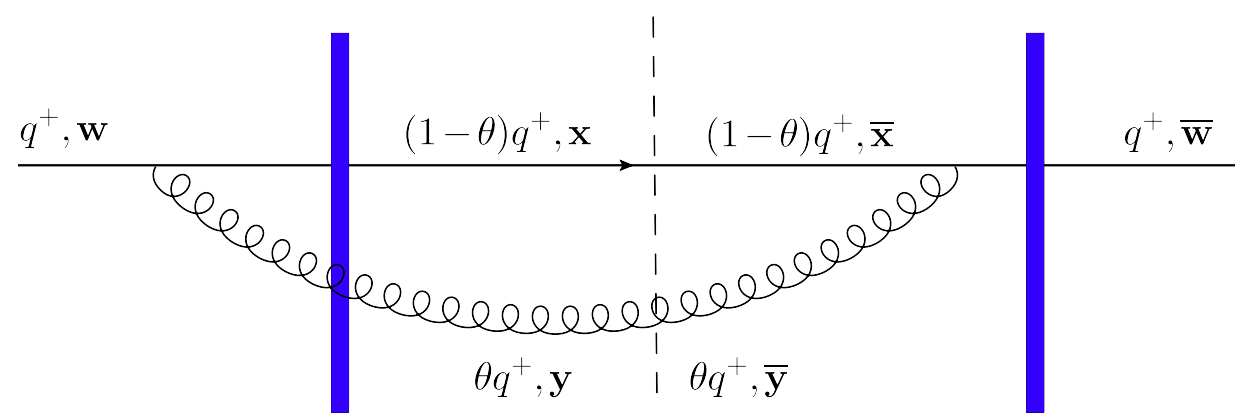

Figure 2. One of the contributions (the one proportional to $S_{q g \bar{q}}(\boldsymbol{x}, \boldsymbol{y}, \overline{\boldsymbol{w}})$ ) to the quark-gluon production cross section in eq. (2.10). There are four such contributions, in which the shockwave can be located either before or after the gluon emission, in both the direct amplitude and the complex conjugate amplitude. The dashed line represents the final state at $x^{+} \rightarrow \infty$.

(for bare partons) over the momentum-space version of the outgoing state. The latter is obtained from eq. (2.4) via a Fourier transform:

$$
\left|q_{\lambda}^{\alpha}\left(q^{+}, \boldsymbol{q}\right)\right\rangle_{q g}^{\text {out }}=\int_{\boldsymbol{w}} \mathrm{e}^{i \boldsymbol{w} \cdot \boldsymbol{q}}\left|q_{\lambda}^{\alpha}\left(q^{+}, \boldsymbol{w}\right)\right\rangle_{q g}^{\text {out }}
$$

Specifically, with our present conventions one can write

$$
\frac{d \sigma_{\mathrm{LO}}^{q A \rightarrow q g+X}}{d^{3} p d^{3} k}(2 \pi) \delta\left(k^{+}+p^{+}-q^{+}\right) \equiv \frac{1}{2 N_{c}} \stackrel{\text { out }}{q g}\left\langle q_{\lambda}^{\alpha}\left(q^{+}, \boldsymbol{q}\right)\left|\hat{\mathcal{N}}_{q}(p) \hat{\mathcal{N}}_{g}(k)\right| q_{\lambda}^{\alpha}\left(q^{+}, \boldsymbol{q}\right)\right\rangle_{q g}^{\text {out }}
$$

Our definitions for the number density operators for bare quarks, $\hat{\mathcal{N}}_{q}(p)$, and for bare gluons, $\hat{\mathcal{N}}_{g}(k)$, are exhibited in eq. (A.17) and respectively (A.19) from appendix A.

As already mentioned, we can choose $\boldsymbol{q}=0$ (the longitudinal axis is taken to be parallel to the collision axis). After some simple algebra, one finds the following result for 
the forward quark-gluon production cross section at LO [1, 49]:

$$
\begin{aligned}
\frac{d \sigma_{\mathrm{LO}}^{q A \rightarrow q g+X}}{d p^{+} d^{2} \boldsymbol{p} d k^{+} d^{2} \boldsymbol{k}}= & \frac{2 \alpha_{s} C_{F}\left[1+(1-\theta)^{2}\right]}{(2 \pi)^{6} \theta q^{+}} \delta\left(q^{+}-k^{+}-p^{+}\right) \\
& \times \int_{\boldsymbol{x}, \overline{\boldsymbol{x}}, \boldsymbol{y}, \overline{\boldsymbol{y}}} \frac{(\boldsymbol{x}-\boldsymbol{y}) \cdot(\overline{\boldsymbol{x}}-\overline{\boldsymbol{y}})}{(\boldsymbol{x}-\boldsymbol{y})^{2}(\overline{\boldsymbol{x}}-\overline{\boldsymbol{y}})^{2}} \mathrm{e}^{-i \boldsymbol{p} \cdot(\boldsymbol{x}-\overline{\boldsymbol{x}})-i \boldsymbol{k} \cdot(\boldsymbol{y}-\overline{\boldsymbol{y}})} \\
& \left.\times\left[S_{q g \bar{q} g}(\boldsymbol{x}, \boldsymbol{y}, \overline{\boldsymbol{x}}, \overline{\boldsymbol{y}})-S_{q g \bar{q}}(\boldsymbol{x}, \boldsymbol{y}, \overline{\boldsymbol{w}})\right)-S_{q \bar{q} g}(\boldsymbol{w}, \overline{\boldsymbol{x}}, \overline{\boldsymbol{y}})+\mathcal{S}(\boldsymbol{w}, \overline{\boldsymbol{w}})\right],
\end{aligned}
$$

with $C_{F} \equiv t^{a} t^{a}=\frac{N_{c}^{2}-1}{2 N_{c}}$ and a compact notation for the transverse integrations: $\int_{\boldsymbol{x}} \equiv \int d^{2} \boldsymbol{x}$. It is understood that $\theta=k^{+} / q^{+}, \boldsymbol{w}=(1-\theta) \boldsymbol{x}+\theta \boldsymbol{y}$ and $\overline{\boldsymbol{w}}=(1-\theta) \overline{\boldsymbol{x}}+\theta \overline{\boldsymbol{y}}$. To obtain this result, we have also used the identity $\varepsilon^{i l} \varepsilon^{j k}=\delta^{i j} \delta^{k l}-\delta^{i k} \delta^{j l}$ to perform the sums over the quark helicities and the gluon polarisations (recall eq. (2.5)):

$$
\phi_{\lambda_{1} \lambda}^{i j \dagger}(\theta) \phi_{\lambda_{1} \lambda}^{i k}(\theta)=4 \delta^{j k}\left(1+(1-\theta)^{2}\right) .
$$

In writing eq. (2.10), we have also performed the average over the random colour fields $A^{-}$describing the soft gluons from the target and we have introduced the following $S$-matrices describing the forward scattering of colourless systems made with up to four partons: a quark-antiquark dipole,

$$
\mathcal{S}(\boldsymbol{w}, \overline{\boldsymbol{w}}) \equiv \frac{1}{N_{c}}\left\langle\operatorname{tr}\left(V(\boldsymbol{w}) V^{\dagger}(\overline{\boldsymbol{w}})\right)\right\rangle,
$$

a quark-antiquark-gluon triplet (this is illustrated in figure 2),

$$
\begin{aligned}
S_{q g \bar{q}}(\boldsymbol{x}, \boldsymbol{y}, \overline{\boldsymbol{w}}) & \equiv \frac{1}{C_{F} N_{c}}\left\langle\operatorname{tr}\left(V^{\dagger}(\overline{\boldsymbol{w}}) t^{b} V(\boldsymbol{x}) t^{a}\right) U^{b a}(\boldsymbol{y})\right\rangle \\
& =\frac{1}{2 C_{F} N_{c}}\left(N_{c}^{2} \mathcal{S}(\boldsymbol{x}, \boldsymbol{y}) \mathcal{S}(\boldsymbol{y}, \overline{\boldsymbol{w}})-\mathcal{S}(\boldsymbol{x}, \overline{\boldsymbol{w}})\right) \simeq \mathcal{S}(\boldsymbol{x}, \boldsymbol{y}) \mathcal{S}(\boldsymbol{y}, \overline{\boldsymbol{w}}),
\end{aligned}
$$

and finally a quark-antiquark pair accompanied by two gluons:

$$
\begin{aligned}
& S_{q g \bar{q} g}(\boldsymbol{x}, \boldsymbol{y}, \overline{\boldsymbol{x}}, \overline{\boldsymbol{y}}) \equiv \frac{1}{C_{F} N_{c}}\left\langle\operatorname{tr}\left(V^{\dagger}(\overline{\boldsymbol{x}}) V(\boldsymbol{x}) t^{a} t^{c}\right)\left[U^{\dagger}(\overline{\boldsymbol{y}}) U(\boldsymbol{y})\right]^{c a}\right\rangle \\
& =\frac{1}{2 C_{F} N_{c}}\left(N_{c}^{2} \mathcal{Q}(\boldsymbol{x}, \boldsymbol{y}, \overline{\boldsymbol{y}}, \overline{\boldsymbol{x}}) \mathcal{S}(\boldsymbol{y}, \overline{\boldsymbol{y}})-\mathcal{S}(\boldsymbol{x}, \overline{\boldsymbol{x}})\right) \simeq \mathcal{Q}(\boldsymbol{x}, \boldsymbol{y}, \overline{\boldsymbol{y}}, \overline{\boldsymbol{x}}) \mathcal{S}(\boldsymbol{y}, \overline{\boldsymbol{y}}) .
\end{aligned}
$$

The second equalities in the r.h.s. of eqs. (2.14) and (2.13) are obtained after using eq. (2.7) together with standard Fierz identities. Besides the colour dipole already introduced in eq. (2.12) they also involve the quadrupole,

$$
\mathcal{Q}(\boldsymbol{x}, \boldsymbol{y}, \overline{\boldsymbol{y}}, \overline{\boldsymbol{x}}) \equiv \frac{1}{N_{c}}\left\langle\operatorname{tr}\left(V(\boldsymbol{x}) V^{\dagger}(\boldsymbol{y}) V(\overline{\boldsymbol{y}}) V^{\dagger}(\overline{\boldsymbol{x}})\right)\right\rangle .
$$

The final, approximate, equalities in eqs. (2.14) and (2.13) hold in the multi-colour limit $N_{c} \rightarrow \infty$, which allows for important simplifications, as already visible in the above results.

It is understood that the target averages occurring in the above equations (2.12)(2.15) are computed over the nuclear gluon distribution evolved down to a longitudinal 
momentum fraction $x_{g}$, cf. eq. (2.1). So, in principle, these $S$-matrices must be obtained from solutions to the non-linear B-JIMWLK equations, which in general form an infinite hierarchy. At large $N_{c}$, one can use simpler equations, which are closed: the BK equation for the dipole $S$-matrix together with the evolution equation for the quadrupole obtained in [71-73]. The latter is still cumbersome to use in practice, so it is useful to notice that a mean field approximation relating the quadrupole to the dipole [51, 54, 72-74] appears to work quite well, even for finite $N_{c}$ [75].

Given the partonic cross-section (2.10), the contribution of the quark channel to the leading-order cross-section for dijet production in $p A$ collisions at forward rapidity is obtained as

$$
\left.\frac{d \sigma_{\mathrm{LO}}^{p A \rightarrow 2 j e t+X}}{d^{3} p d^{3} k}\right|_{q-\text { channel }}=\int d x_{q} q_{f}\left(x_{q}, \mu^{2}\right) \frac{d \sigma_{\mathrm{LO}}^{q A \rightarrow q g+X}}{d^{3} p d^{3} k},
$$

where $q_{f}\left(x_{q}, \mu^{2}\right)$ is the quark distribution function of the proton, for a longitudinal momentum fraction $x_{q}=q^{+} / Q^{+}$and a resolution scale $\mu^{2}$. At LO, the value of $x_{q}$ is fixed by the $\delta$-function in eq. (2.10). Furthermore, $\mu^{2}$ should be chosen of the order of the hardest among the transverse momenta, $\boldsymbol{k}^{2}$ or $\boldsymbol{p}^{2}$, of the produced jets, or hadrons. ${ }^{2}$ The final result at $\mathrm{LO}$ and for large $N_{c}$ reads

$$
\begin{aligned}
\frac{d \sigma_{\mathrm{LO}}^{p A \rightarrow q g+X}}{d^{3} p d^{3} k}= & x_{q} q_{f}\left(x_{q}, \mu^{2}\right) \frac{\bar{\alpha}_{s}}{(2 \pi)^{5}} \frac{1+(1-\theta)^{2}}{2 \theta\left(q^{+}\right)^{2}} \\
& \times \int_{\boldsymbol{x}, \overline{\boldsymbol{x}}, \boldsymbol{y}, \overline{\boldsymbol{y}}} \frac{(\boldsymbol{x}-\boldsymbol{y}) \cdot(\overline{\boldsymbol{x}}-\overline{\boldsymbol{y}})}{(\boldsymbol{x}-\boldsymbol{y})^{2}(\overline{\boldsymbol{x}}-\overline{\boldsymbol{y}})^{2}} \mathrm{e}^{-i \boldsymbol{p} \cdot(\boldsymbol{x}-\overline{\boldsymbol{x}})-i \boldsymbol{k} \cdot(\boldsymbol{y}-\overline{\boldsymbol{y}})} \\
& \times[\mathcal{Q}(\boldsymbol{x}, \boldsymbol{y}, \overline{\boldsymbol{y}}, \overline{\boldsymbol{x}}) \mathcal{S}(\boldsymbol{y}, \overline{\boldsymbol{y}})-\mathcal{S}(\boldsymbol{x}, \boldsymbol{y}) \mathcal{S}(\boldsymbol{y}, \overline{\boldsymbol{w}})-\mathcal{S}(\boldsymbol{w}, \overline{\boldsymbol{y}}) \mathcal{S}(\overline{\boldsymbol{y}}, \overline{\boldsymbol{x}})+\mathcal{S}(\boldsymbol{w}, \overline{\boldsymbol{w}})],
\end{aligned}
$$

where $q^{+}=p^{+}+k^{+}, \theta=k^{+} / q^{+}$, and $x_{q}=q^{+} / Q^{+}$.

We shall later need also the expression of the $\mathrm{LO}$ cross-section for the case where one is measuring a pair of hadrons (instead of jets) in the final state. Under the assumption that the parton $\rightarrow$ hadron splitting is collinear, this cross-section is obtained by convoluting eq. (2.17) with the quark $\rightarrow$ hadron and gluon $\rightarrow$ hadron fragmentation functions:

$$
\begin{aligned}
& \left.\frac{d \sigma_{\mathrm{LO}}^{p A \rightarrow h_{1} h_{2}+X}}{d^{3} p d^{3} k}\right|_{q-\text { channel }} \\
& \quad=\int \frac{d z_{1}}{z_{1}^{3}} \int \frac{d z_{2}}{z_{2}^{3}} \int d x_{q} q_{f}\left(x_{q}, \mu^{2}\right) \frac{d \sigma_{\mathrm{LO}}^{q A \rightarrow q g+X}}{d^{3} p_{1} d^{3} k_{1}} D_{h_{1} / q}\left(z_{1}, \mu^{2}\right) D_{h_{2} / g}\left(z_{2}, \mu^{2}\right),
\end{aligned}
$$

where it is understood that $p_{1}=p / z_{1}, k_{1}=k / z_{2}$ (for both longitudinal and transverse components), $x_{q}=\left(p_{1}^{+}+k_{1}^{+}\right) / Q^{+}$, and $\theta=k_{1}^{+} /\left(p_{1}^{+}+k_{1}^{+}\right)$. This expression (2.18) for the dijet cross-section is a particular example of the hybrid factorisation, originally introduced in the context of single hadron production in $p A$ collisions [2-4]. Thus is "hybrid" in the sense that the initial and final states are treated in the spirit of the collinear factorisation, whereas the hard process is rather computed using the CGC effective theory for QCD

\footnotetext{
${ }^{2}$ As we shall discuss in section 6 , this particular choice minimises the magnitude of the NLO corrections associated with the DGLAP evolution of the quark distribution in the proton.
} 
at high energy (which in turn can be viewed as a generalisation of the $k_{T}$-factorisation originally developed in the context of the BFKL evolution $[44,45])$.

Before we proceed with the calculation of NLO corrections to the partonic cross-section in eq. (2.10), let us briefly comment on another type of corrections, which are not suppressed by powers of the QCD coupling but rather by inverse powers of the center-of-mass energy $\sqrt{s}$, and which could become important already at leading order in $\alpha_{s}$ if this energy is not high enough: these are corrections to the eikonal approximation underlying the partonic $S$-matrices which appear in eq. (2.10). As discussed in relation with eq. (2.6), this approximation amounts to neglecting the change in the transverse position of the projectile parton associated with its scattering. This assumption is justified so long as the coherence time $\tau \equiv 2 k^{+} / \boldsymbol{k}^{2}$ of that parton is much larger than the longitudinal extent $L=2 R_{A} / \gamma$ of the nuclear target. ${ }^{3}$ Here, $k^{+}$and $\boldsymbol{k}$ are the parton longitudinal and transverse momenta, respectively, $R_{A} \simeq A^{1 / 3} R$ (with $R$ the proton radius) is the nuclear radius in its rest frame , and $\gamma=P^{-} / M$ is the Lorentz contraction factor ( $M$ is the nucleon mass and $P^{-}$its longitudinal momentum). Corrections to the eikonal approximation are suppressed by powers of the "small parameter" $L / \tau$, estimated as

$$
\frac{L}{\tau}=\frac{k_{\perp}^{2}}{2 k^{+}} \frac{2 R_{A} M}{P^{-}}=\frac{k_{\perp}^{2}}{x s} 2 R_{A} M=\frac{k_{\perp}}{\sqrt{s}} \mathrm{e}^{-\eta} 2 R_{A} M
$$

where $x=k^{+} / Q^{+}, s=2 Q^{+} P^{-}$, and the last equality holds in the center-of-mass frame, where $x$ can be related to the parton rapidity $\eta$ via $x=\left(k_{\perp} / \sqrt{s}\right) \mathrm{e}^{\eta}$ (recall eq. (2.2)). For numerical estimates, we shall use $A^{1 / 3}=6, R=1 \mathrm{fm}, M=1 \mathrm{GeV}$, together with $k_{\perp}=$ $2 \mathrm{GeV}$ (which is of the order of the nuclear saturation momentum $Q_{s}$ ); hence $2 R_{A} M k_{\perp} \simeq$ $120 \mathrm{GeV}$. At the LHC energies, $\sqrt{s} \geq 2 \mathrm{TeV}$, this ratio $L / \tau$ is very small for any $\eta \geq 0$, that is, at both central $(\eta \simeq 0)$ and forward $(\eta \geq 1)$ rapidities. At RHIC on the other hand, where $\sqrt{s}=200 \mathrm{GeV}$, the condition $L / \tau \ll 1$ is satisfied only for sufficiently forward rapidities, say $\eta>1$. Throughout this paper, we assume that this condition is well satisfied, hence the eikonal approximation is indeed justified, at least at leading order. That said, the sub-eikonal corrections are expected to compete with the NLO perturbative corrections whenever $L / \tau \sim \mathcal{O}\left(\alpha_{s}\right)$. Within the LO CGC formalism, such corrections to (multi)particle production in dilute-dense collisions have been studied in [76-80] (and references therein).

\section{Trijet cross-section at leading order}

In preparation for the calculation of the real NLO corrections to dijet production, let us first revisit our results for the LO trijet ( $q q \bar{q}$ and $q g g$ ) cross-sections, originally presented in ref. [1]. As compared to [1], we shall improve these results at two levels: (i) we shall rewrite them in a different way, which is not only more compact and physically more transparent, but also better suited for the purposes of the NLO calculation, and (ii) we shall correct

\footnotetext{
${ }^{3} \mathrm{~A}$ simple way to understand this condition is as follows: in order to be negligible, the transverse deviation $\Delta x_{\perp} \sim\left(k_{\perp} / k^{+}\right) L$ generated by a scattering with transferred momentum $k_{\perp}$ must be much smaller than the quantum uncertainty $\sim 1 / k_{\perp}$ in the transverse position of the parton.
} 
several errors and misprints, that we failed to identify in ref. [1], by lack of appropriate tests. Hence, the results for the trijet cross-section to be presented in this section should be viewed as our final respective results, in replacement of those in ref. [1]. And as matter of facts, we have obtained these new results via an independent calculation, and not by just reshuffling terms in the original results from ref. [1].

\subsection{The tri-parton components of the quark outgoing state}

We first present the expressions of the tri-parton Fock-space components of the quark outgoing state. These are obtained by expanding the QCD evolution operators in $|q\rangle^{\text {out }}=$ $U_{I}(\infty, 0) \hat{S} U_{I}(0,-\infty)|q\rangle^{\text {in }}$ to second order in the interaction Hamiltonian (see e.g. ref. [1] for details). In this expansion, we shall ignore the virtual corrections for the time being (they will be computed in a subsequent paper); that is, we shall only keep those secondorder terms which contribute to a tri-parton $(q q \bar{q}$ and $q g g)$ final state. We shall not present the details of the calculations - they would be very similar to those described at length in ref. [1] — but only emphasise the differences w.r.t. [1], which mainly refer to a reshuffling of terms. For the convenience of the reader, our conventions for the light-cone wavefunction formalism are summarised in appendix A, whereas appendix B exhibits all the matrix elements of the QCD interaction Hamiltonian which are relevant for this calculation.

\subsubsection{The tri-quark final state}

To explain this reshuffling, we shall focus on the three-quark $(q q \bar{q})$ final state. As explained in [1], this state receives two types of contributions,

$$
\left|q_{\lambda}^{\alpha}\right\rangle_{q q \bar{q}}^{\text {out }}=\left|q_{\lambda}^{\alpha}\right\rangle_{q q \bar{q}}^{r e g}+\left|q_{\lambda}^{\alpha}\right\rangle_{q q \bar{q}}^{\text {inst }}
$$

where the second term, with upper-script inst, refers to the instantaneous piece of the intermediate gluon propagator in the LC gauge, where the first term with upper-script reg refers to its regular piece, which is non-local in LC time (see also figures 3 and 5).

The general expression for the regular piece, as obtained to second order in light-cone perturbation theory, reads (see eq. (4.2) in [1])

$$
\begin{aligned}
& \left|q_{\lambda}^{\alpha}\right\rangle_{q q \bar{q}}^{r e g} \\
& \equiv \frac{1}{2}\left|\bar{q}_{\lambda_{3}}^{\rho} q_{\lambda_{2}}^{\varrho} q_{\lambda_{1}}^{\sigma}\right\rangle\left\{\frac{\left\langle\bar{q}_{\lambda_{3}}^{\rho} q_{\lambda_{2}}^{\varrho} q_{\lambda_{1}}^{\sigma}|\hat{S}| \bar{q}_{\lambda_{7}}^{\delta} q_{\lambda_{6}}^{\epsilon} q_{\lambda_{5}}^{\kappa}\right\rangle\left\langle\bar{q}_{\lambda_{7}}^{\delta} q_{\lambda_{6}}^{\epsilon} q_{\lambda_{5}}^{\kappa}\left|\mathrm{H}_{g \rightarrow q \bar{q}}\right| q_{\lambda_{4}}^{\beta} g_{i}^{a}\right\rangle\left\langle q_{\lambda_{4}}^{\beta} g_{i}^{a}\left|\mathrm{H}_{q \rightarrow q g}\right| q_{\lambda}^{\alpha}\right\rangle}{\left(E_{q q \bar{q}}-E_{q}\right)\left(E_{q g}-E_{q}\right)}\right. \\
& \left.+\frac{\left\langle\bar{q}_{\lambda_{3}}^{\rho} q_{\lambda_{2}}^{\varrho} q_{\lambda_{1}}^{\sigma}\left|\mathrm{H}_{g \rightarrow q \bar{q}}\right| q_{\lambda_{5}}^{\gamma} g_{i}^{a}\right\rangle\left\langle q_{\lambda_{5}}^{\gamma} g_{i}^{a}\left|\mathrm{H}_{q \rightarrow q g}\right| q_{\lambda_{4}}^{\beta}\right\rangle\left\langle q_{\lambda_{4}}^{\beta}|\hat{S}| q_{\lambda}^{\alpha}\right\rangle}{\left(E_{q g}-E_{q q \bar{q}}\right)\left(E_{q}-E_{q q \bar{q}}\right)}\right\}, \\
& \left.\quad+\frac{\left\langle\bar{q}_{\lambda_{3}}^{\rho} q_{\lambda_{2}}^{\varrho} q_{\lambda_{1}}^{\sigma}\left|\mathrm{H}_{g \rightarrow q \bar{q}}\right| q_{\lambda_{5}}^{\gamma} g^{j}\right\rangle\left\langle q_{\lambda_{5}}^{\gamma} g^{j}|\hat{S}| q_{\lambda_{4}}^{\beta} g_{i}^{a}\right\rangle\left\langle q_{\lambda_{4}}^{\beta} g_{i}^{a}\left|\mathrm{H}_{q \rightarrow q g}\right| q_{\lambda}^{\alpha}\right\rangle}{\left(E_{q g}-E_{q q \bar{q}}\right)\left(E_{q g}-E_{q}\right)}\right\},
\end{aligned}
$$

The three terms in the r.h.s. of this equation correspond to the different possible insertions of the shockwave relative to the two parton branchings, as illustrated by the Feynman 


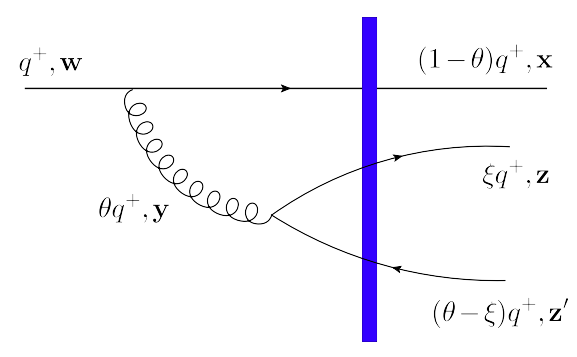

(a)

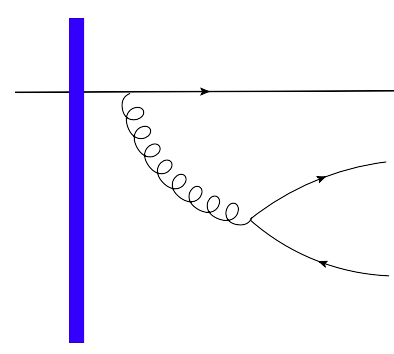

(b)

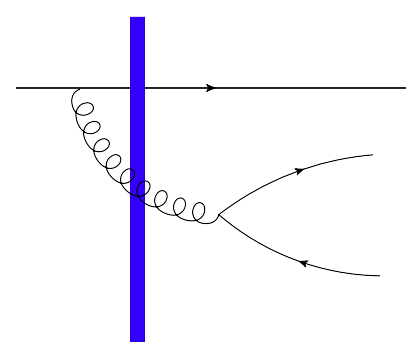

(c)

Figure 3. The three possible configurations for the interplay between parton branching and scattering, for a final partonic state built with three quarks: (a) initial-state evolution (the first term in the r.h.s. of eq. (3.2)), (b) mixed evolution: the gluon is emitted prior to the scattering, but it splits after the scattering (the second term in eq. (3.2)), (c) final-state evolution (the third term in eq. $(3.2))$.

graphs in figure 3. In the first term, cf. figure 3.a, both parton branchings occur prior to the scattering off the shockwave, hence they are generated by the second-order expansion of the evolution operator $U_{I}(0,-\infty)$. Accordingly, both energy denominators involve differences w.r.t. the light-cone energy $E_{q}$ of the initial state. Similarly, the second term, cf. figure 3.b, describes the process where both branchings occur in the final state (after the scattering), hence they are generated by $U_{I}(\infty, 0)$. The corresponding energy denominators involve differences w.r.t. the energy $E_{q q \bar{q}}$ of the final state. Finally, the third term, cf. figure 3.c, describes one emission prior to the scattering, as generated by the first order term in $U_{I}(0,-\infty)$, and a second one after the scattering - the first order term in $U_{I}(\infty, 0)$.

The reshuffling of terms is based on the following identity:

$$
\frac{1}{\left(E_{q g}-E_{q q \bar{q}}\right)\left(E_{q}-E_{q q \bar{q}}\right)}=-\frac{1}{\left(E_{q q \bar{q}}-E_{q}\right)\left(E_{q g}-E_{q}\right)}-\frac{1}{\left(E_{q g}-E_{q q \bar{q}}\right)\left(E_{q g}-E_{q}\right)},
$$

which has a simple physical meaning: it shows that in the absence of scattering (i.e. in the limit $\hat{S} \rightarrow 1$ ), the three second-order corrections in eq. (3.2) exactly cancel each other. In turn, this is a consequence of the fact that an on-shell quark cannot radiate gluons in the absence of any scattering (see the discussion in [1]). In what follows, we shall rely on this identity to re-express the middle term in eq. (3.2), cf. figure 3.b, as the sum of two negative contributions which exhibit the same energy denominators as the two other terms there (see figure 4 for a graphical illustration of this manipulation). After this rewriting, the contribution of the initial-state scattering is treated as a subtraction term for the two other contributions (those shown in figures 3.a and 3.c).

It is in principle straightforward to implement this reshuffling of terms via manipulations of the original results for the trijet final state, as presented in [1]. However, in presenting our respective results in what follows, we shall often use different integration variables compared to [1]. Moreover, as stated at the beginning of this section, our original results in [1] contained some misprints and errors, that we have identified (and corrected) after explicitly working out special limits. In view of that, it is perhaps not so useful to compare with ref. [1] anymore, but simply rely on the new results to be presented here. 


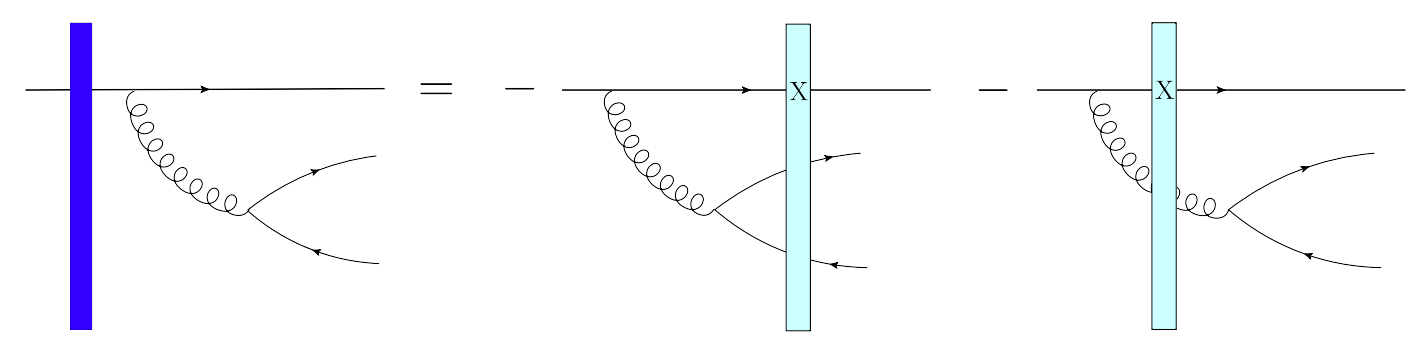

Figure 4. The new way in which we express the contribution of the initial-state interaction (the middle term in eq. (3.2)) after using the identity eq. (3.3). The light-colour shockwave in the r.h.s. represent the $S$-matrix (a Wilson line) for the scattering of only one parton: the original quark, denoted with a cross.

This is in particular true for the $q q \bar{q}$ component of the outgoing quark state. In ref. [1], this has been obtained by separately evaluating the three terms in the r.h.s. of eq. (3.2), with the result shown in eq. (4.9) of that paper. After treating the second term there (which corresponds to figure 3.b) as a subtraction term, performing some changes of variables, and correcting an error, one finds

$$
\begin{aligned}
& \left|q_{\lambda}^{\alpha}\left(q^{+}, \boldsymbol{w}\right)\right\rangle_{q q \bar{q}}^{r e g}=\frac{g^{2} q^{+}}{2(2 \pi)^{4}} \int_{\boldsymbol{x}, \boldsymbol{z}, \boldsymbol{z}^{\prime}} \int_{0}^{1} d \theta \int_{0}^{\theta} d \xi \varphi_{\lambda_{2} \lambda_{3}}^{i l}\left(\frac{\xi}{\theta}\right) \phi_{\lambda_{1} \lambda}^{i j}(\theta) \frac{\theta^{2}(1-\theta) \boldsymbol{Z}^{l} \boldsymbol{R}^{j}}{\boldsymbol{Z}^{2}} \\
& \times\left[\frac{V^{\varrho \delta}(\boldsymbol{z}) t_{\delta \epsilon}^{a} V^{\dagger \epsilon \rho}\left(\boldsymbol{z}^{\prime}\right) V^{\sigma \beta}(\boldsymbol{x}) t_{\beta \alpha}^{a}-t_{\varrho \rho}^{a} t_{\sigma \beta}^{a} V^{\beta \alpha}(\boldsymbol{w})}{\theta^{2}(1-\theta) \boldsymbol{R}^{2}+\xi(\theta-\xi) \boldsymbol{Z}^{2}}-\frac{t_{\varrho \rho}^{b} U^{b a}(\boldsymbol{y}) V^{\sigma \beta}(\boldsymbol{x}) t_{\beta \alpha}^{a}-t_{\varrho \rho}^{b} t_{\sigma \beta}^{b} V^{\beta \alpha}(\boldsymbol{w})}{\theta^{2}(1-\theta) \boldsymbol{R}^{2}}\right] \\
& \times \delta^{(2)}((1-\theta) \boldsymbol{x}+\theta \boldsymbol{y}-\boldsymbol{w})\left|\bar{q}_{\lambda_{3}}^{\rho}\left((\theta-\xi) q^{+}, \boldsymbol{z}^{\prime}\right) q_{\lambda_{2}}^{\varrho}\left(\xi q^{+}, \boldsymbol{z}\right) q_{\lambda_{1}}^{\sigma}\left((1-\theta) q^{+}, \boldsymbol{x}\right)\right\rangle .
\end{aligned}
$$

Our notations are illustrated in figure 3): $\theta$ and $\xi$ are the longitudinal momentum fractions (w.r.t. the momentum $q^{+}$of the incoming quark) of the intermediate gluon and of the quark produced by the splitting $g \rightarrow q \bar{q}$, respectively. Also, $\boldsymbol{x}$ and $\boldsymbol{y}$ are the transverse coordinates of the quark and the emitted gluon, whereas $\boldsymbol{z}$ and $\boldsymbol{z}^{\prime}$ similarly refer to the quark and the antiquark generated by the gluon. Notice that $\boldsymbol{y}$ is not an independent coordinate, since it must coincide with the center of energy of the $q \bar{q}$ pair produced by the gluon decay. Similarly, the transverse coordinate $\boldsymbol{w}$ of the incoming quark must be the same as the center of energy of the quark-gluon pair in the intermediate state, or of the three quarks in the final state. Explicitly,

$$
\boldsymbol{y} \equiv \frac{\xi \boldsymbol{z}+(\theta-\xi) \boldsymbol{z}^{\prime}}{\theta}, \quad \boldsymbol{w}=(1-\theta) \boldsymbol{x}+\theta \boldsymbol{y}=(1-\theta) \boldsymbol{x}+\xi \boldsymbol{z}+(\theta-\xi) \boldsymbol{z}^{\prime}
$$

The $\delta$-function inside the integrand enforces the condition on $\boldsymbol{w}$ shown in the second equality in eq. (3.5), thus introducing a constraint on the 5 integrations visible in the r.h.s. of eq. (3.4). The transverse coordinates denoted with capital letters, that is,

$$
\boldsymbol{R} \equiv \boldsymbol{x}-\boldsymbol{y}, \quad \boldsymbol{Z} \equiv \boldsymbol{z}-\boldsymbol{z}^{\prime}
$$

are the transverse separations between the daughter partons after each of the emission vertices. Finally, the spinorial structure of the two branching vertices is encoded in the 
following tensors:

$$
\begin{aligned}
& \phi_{\lambda_{1} \lambda}^{i j}(\theta) \equiv \chi_{\lambda_{1}}^{\dagger}\left[(2-\theta) \delta^{i j}-i \theta \varepsilon^{i j} \sigma^{3}\right] \chi_{\lambda}=\delta_{\lambda \lambda_{1}}\left[(2-\theta) \delta^{i j}-2 i \theta \varepsilon^{i j} \lambda\right] \\
& \varphi_{\lambda_{1} \lambda}^{i j}(\xi) \equiv \chi_{\lambda_{1}}^{\dagger}\left[(2 \xi-1) \delta^{i j}+i \varepsilon^{i j} \sigma^{3}\right] \chi_{\lambda}=\delta_{\lambda \lambda_{1}}\left[(2 \xi-1) \delta^{i j}+2 i \varepsilon^{i j} \lambda\right] .
\end{aligned}
$$

The two terms within the square brackets in eq. (3.4) represent the contributions of the two graphs in figure 3.a and figure 3.c respectively, minus the respective subtraction terms generated by the graph in figure 3.b, as shown in figure 4 . Thanks to these subtractions, the numerator in each of these terms vanishes in the limit where the transverse separations between the daughter partons (as appearing in the respective denominator) are shrinking to zero. Consider e.g. the second term: when $\boldsymbol{R}=\boldsymbol{x}-\boldsymbol{y} \rightarrow 0$, meaning that the three coordinates $\boldsymbol{x}, \boldsymbol{y}$ and $\boldsymbol{w}$ become coincident with each other, one can use the following identity (cf. eq. (2.7))

$$
U^{b a}(\boldsymbol{x}) t_{\beta \alpha}^{a}=\left[V^{\dagger}(\boldsymbol{x}) t^{b} V(\boldsymbol{x})\right]_{\beta \alpha},
$$

to verify that the respective numerator vanishes, as announced. Similarly, the numerator in the first line vanishes when both $\boldsymbol{R} \rightarrow 0$ and $\boldsymbol{Z} \rightarrow 0$ (meaning that all transverse coordinates become degenerate: $\boldsymbol{w}=\boldsymbol{x}=\boldsymbol{y}=\boldsymbol{z}=\boldsymbol{z}^{\prime}$ ). Furthermore the two terms within the square brackets in eq. (3.4) cancel each other in the limit $\boldsymbol{Z} \rightarrow 0$ (i.e. $\boldsymbol{z}=\boldsymbol{z}^{\prime}=\boldsymbol{y}$ ) at fixed $\boldsymbol{R}$. To see this, one should use yet another version of the identity eq. (2.7), namely,

$$
\left[V(\boldsymbol{y}) t^{a} V^{\dagger}(\boldsymbol{y})\right]_{\alpha \beta}=t_{\alpha \beta}^{b} U^{b a}(\boldsymbol{y})
$$

which expresses the fact that the scattering cannot distinguish a $q \bar{q}$ fluctuation of zero size from its parent gluon (recall the discussion after eq. (2.7)).

These properties of the LCWF will be later useful in demonstrating the cancellation of "ultraviolet" (short-distance) singularities in the calculation of cross-sections for particle production. Such properties are more difficult to check on the original expression for the LCWF, i.e. eq. (4.9) in ref. [1].

The previous discussion also implies that the second term in eq. (3.4) can be generated from the first term there via the simultaneous replacements $\boldsymbol{z} \rightarrow \boldsymbol{y}$ and $\boldsymbol{z}^{\prime} \rightarrow \boldsymbol{y}$. This allows us to introduce a simpler notation, that we shall systematically use throughout this paper. Specifically, eq. (3.4) can be equivalently written as

$$
\begin{aligned}
& \left|q_{\lambda}^{\alpha}\left(q^{+}, \boldsymbol{w}\right)\right\rangle_{q q \bar{q}}^{r e g}=\frac{g^{2} q^{+}}{2(2 \pi)^{4}} \int_{\boldsymbol{x}, \boldsymbol{z}, \boldsymbol{z}^{\prime}} \int_{0}^{1} d \theta \int_{0}^{\theta} d \xi \varphi_{\lambda_{2} \lambda_{3}}^{i l}\left(\frac{\xi}{\theta}\right) \phi_{\lambda_{1} \lambda}^{i j}(\theta) \frac{\theta(1-\theta) \boldsymbol{R}^{j} \boldsymbol{Z}^{l}}{\boldsymbol{Z}^{2}} \\
& \quad \times\left[\frac{V^{\varrho \delta}(\boldsymbol{z}) t_{\delta \epsilon}^{a} V^{\dagger \epsilon \rho}\left(\boldsymbol{z}^{\prime}\right) V^{\sigma \beta}(\boldsymbol{x}) t_{\beta \alpha}^{a}-t_{\varrho \rho}^{a} t_{\sigma \beta}^{a} V^{\beta \alpha}(\boldsymbol{w})}{\theta^{2}(1-\theta) \boldsymbol{R}^{2}+\xi(\theta-\xi) \boldsymbol{Z}^{2}}-\left(\boldsymbol{z}, \boldsymbol{z}^{\prime} \rightarrow \boldsymbol{y}\right)\right] \\
& \quad \times \delta^{(2)}((1-\theta) \boldsymbol{x}+\theta \boldsymbol{y}-\boldsymbol{w})\left|\bar{q}_{\lambda_{3}}^{\rho}\left((\theta-\xi) q^{+}, \boldsymbol{z}^{\prime}\right) q_{\lambda_{2}}^{\varrho}\left(\xi q^{+}, \boldsymbol{z}\right) q_{\lambda_{1}}^{\sigma}\left((1-\theta) q^{+}, \boldsymbol{x}\right)\right\rangle .
\end{aligned}
$$

Besides this "regular" contribution, which involves an intermediate state with a propagating gluon, there is an additional contribution built with the instantaneous piece of the gluon propagator (see figure 5). This is given by eq. (4.13) in ref. [1], which with the 


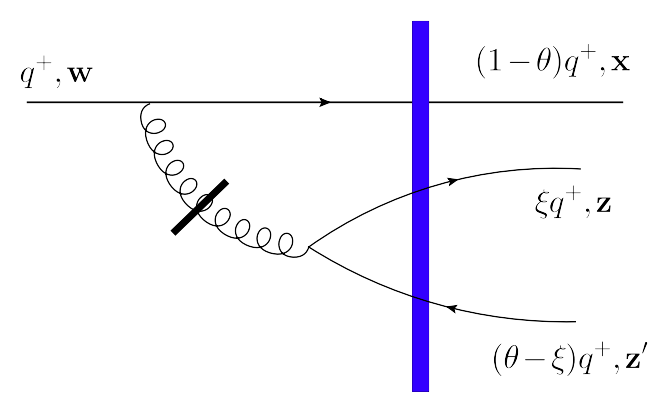

(a)

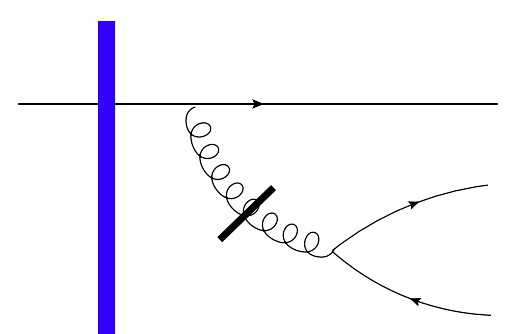

(b)

Figure 5. The two possible insertions of the shockwave in the case where the final quark antiquark pair has been produced via an instantaneous gluon interaction: (a) initial-state evolution, (b) finalstate evolution.

present conventions reads

$$
\begin{aligned}
\left|q_{\lambda}^{\alpha}\left(q^{+}, \boldsymbol{w}\right)\right\rangle_{q q \bar{q}}^{i n s t}= & -\frac{g^{2} q^{+}}{(2 \pi)^{4}} \int_{\boldsymbol{x}, \boldsymbol{z}, \boldsymbol{z}^{\prime}} \int_{0}^{1} d \theta \int_{0}^{\theta} d \xi \frac{(1-\theta) \xi(\theta-\xi)}{\theta\left(\theta^{2}(1-\theta) \boldsymbol{R}^{2}+\xi(\theta-\xi) \boldsymbol{Z}^{2}\right)} \\
& \times\left[V^{\varrho \delta}(\boldsymbol{z}) t_{\delta \epsilon}^{a} V^{\dagger \epsilon \rho}\left(\boldsymbol{z}^{\prime}\right) V^{\sigma \beta}(\boldsymbol{x}) t_{\beta \alpha}^{a}-t_{\varrho \rho}^{a} t_{\sigma \beta}^{a} V^{\beta \alpha}(\boldsymbol{w})\right] \\
& \times \delta^{(2)}(\boldsymbol{w}-\boldsymbol{c})\left|\bar{q}_{\lambda_{3}}^{\rho}\left((\theta-\xi) q^{+}, \boldsymbol{z}^{\prime}\right) q_{\lambda_{2}}^{\varrho}\left(\xi q^{+}, \boldsymbol{z}\right) q_{\lambda_{1}}^{\sigma}\left((1-\theta) q^{+}, \boldsymbol{x}\right)\right\rangle,
\end{aligned}
$$

where $\boldsymbol{c} \equiv(1-\theta) \boldsymbol{x}+\xi \boldsymbol{z}+(\theta-\xi) \boldsymbol{z}^{\prime}$ is a compact notation for the center of energy of the final quark triplet. Note that, in this case, there is no intermediate gluon state: the cut gluon propagator in figure 5 together with the two attached QCD vertices defines a local effective vertex for the decay $q \rightarrow q q \bar{q}$ of the initial quark into the three final ones. So, in particular, there is no analog of the second term in eq. (3.4) (the one denoted as $\left(\boldsymbol{z}, \boldsymbol{z}^{\prime} \rightarrow \boldsymbol{y}\right)$ in eq. (3.10)). That said, it is still convenient to introduce a compact notation (similar to that in eq. (3.10)) for the complete triquark outgoing state, i.e. the sum of the two contributions in eqs. (3.4) and (3.11). This reads

$$
\begin{aligned}
& \left|q_{\lambda}^{\alpha}\left(q^{+}, \boldsymbol{w}\right)\right\rangle_{q q \bar{q}}=\frac{g^{2} q^{+}}{2(2 \pi)^{4}} \int_{\boldsymbol{x}, \boldsymbol{z}, \boldsymbol{z}^{\prime}} \int_{0}^{1} d \theta \int_{0}^{\theta} d \xi \frac{\boldsymbol{R}^{j} \boldsymbol{Z}^{l}}{\boldsymbol{Z}^{2}} \\
& \quad \times\left[\Phi_{\lambda_{3} \lambda_{2} \lambda_{1} \lambda}^{j l} \frac{V^{\varrho \delta}(\boldsymbol{z}) t_{\delta \epsilon}^{a} V^{\dagger \epsilon \rho}\left(\boldsymbol{z}^{\prime}\right) V^{\sigma \beta}(\boldsymbol{x}) t_{\beta \alpha}^{a}-t_{\varrho \rho}^{a} t_{\sigma \beta}^{a} V^{\beta \alpha}(\boldsymbol{w})}{\theta^{2}(1-\theta) \boldsymbol{R}^{2}+\xi(\theta-\xi) \boldsymbol{Z}^{2}}-\left(\boldsymbol{z}, \boldsymbol{z}^{\prime} \rightarrow \boldsymbol{y}\right)\right] \\
& \quad \times \delta^{(2)}(\boldsymbol{w}-\boldsymbol{c})\left|\bar{q}_{\lambda_{3}}^{\rho}\left((\theta-\xi) q^{+}, \boldsymbol{z}^{\prime}\right) q_{\lambda_{2}}^{\varrho}\left(\xi q^{+}, \boldsymbol{z}\right) q_{\lambda_{1}}^{\sigma}\left((1-\theta) q^{+}, \boldsymbol{x}\right)\right\rangle .
\end{aligned}
$$

where $\Phi_{\lambda_{3} \lambda_{2} \lambda_{1} \lambda}^{j l} \equiv \Phi_{\lambda_{3} \lambda_{2} \lambda_{1} \lambda}^{j l}\left(\boldsymbol{x}, \boldsymbol{z}, \boldsymbol{z}^{\prime}, \theta, \xi\right)$ is an effective vertex for the splitting of the original quark into the three final quarks, which includes contributions from both propagating and instantaneous intermediate gluons:

$\Phi_{\lambda_{3} \lambda_{2} \lambda_{1} \lambda}^{j l}\left(\boldsymbol{x}, \boldsymbol{z}, \boldsymbol{z}^{\prime}, \theta, \xi\right) \equiv \theta(1-\theta) \varphi_{\lambda_{2} \lambda_{3}}^{i l}\left(\frac{\xi}{\theta}\right) \phi_{\lambda_{1} \lambda}^{i j}(\theta)-\delta_{\lambda_{2} \lambda_{3}} \delta_{\lambda_{1} \lambda} \delta^{j l} \frac{2(1-\theta) \xi(\theta-\xi)}{\theta} \frac{\boldsymbol{Z}^{2}}{\boldsymbol{R} \cdot \boldsymbol{Z}}$. 


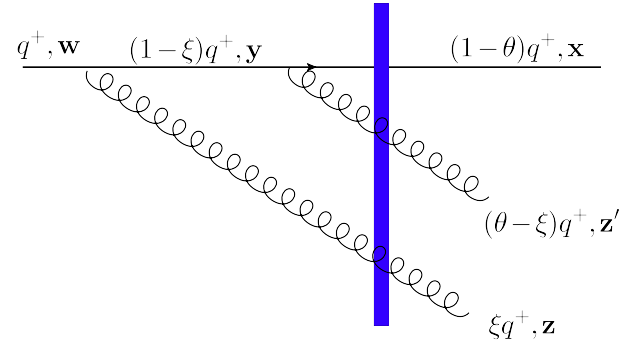

(a)

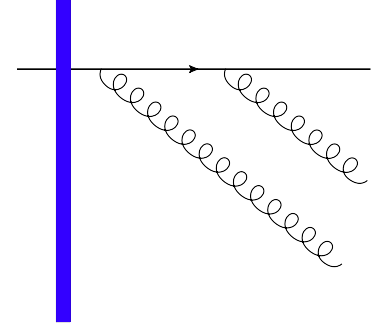

(b)

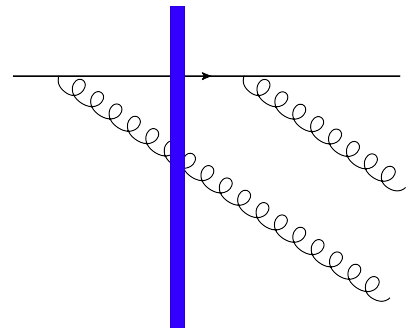

(c)

Figure 6. The three possible configurations for the interplay between parton branching and scattering, for a final partonic state built with three quarks: (a) initial-state evolution (the first term in the r.h.s. of eq. (3.2)), (b) mixed evolution: the gluon is emitted prior to the scattering, but it splits after the scattering (the second term in eq. (3.2)), (c) final-state evolution (the third term in eq. (3.2)).

It is understood that the second piece in eq. (3.13) vanishes after substituting $\boldsymbol{z} \rightarrow \boldsymbol{y}$ and $z^{\prime} \rightarrow y:$

$$
\Phi_{\lambda_{3} \lambda_{2} \lambda_{1} \lambda}^{j l}(\boldsymbol{x}, \boldsymbol{y}, \boldsymbol{y}, \theta, \xi)=\theta(1-\theta) \varphi_{\lambda_{2} \lambda_{3}}^{i l}\left(\frac{\xi}{\theta}\right) \phi_{\lambda_{1} \lambda}^{i j}(\theta)
$$

Hence the subtraction of the contribution from the intermediate gluon state is truly performed only for the case of a propagating gluon, as it should. Ultimately, one should keep in mind that the compact expression for the three-quark outgoing state in eq. (3.12) is merely a convenient notation, which is rather formal, but will be useful to simplify the writing for our final results.

\subsubsection{The final state with one quark and two gluons}

In this subsection we shall exhibit the remaining 3-parton Fock space components of the quark outgoing LCWF: those in which the original quark is accompanied by two gluons. There are two possible topologies for the gluon emissions, as shown in figures 6 and 7 , respectively. Besides these "regular" graphs, where the intermediate parton (quark or gluon) is propagating, there are similar graphs which involve the instantaneous piece of the respective propagator. (These are not shown, but can be easily inferred by comparing with figure 5.) All these graphs have been computed in [1], with results that we shall here rewrite by regrouping terms and also correct whenever necessary, as explained in the previous subsection on the example of the three-quark final state.

We start with the graphs where both gluons are emitted by the original quark, cf. figure 6. Their contribution is shown in eq. (4.14) of [1], that can be rewritten as

$$
\begin{aligned}
\left|q_{\lambda}^{\alpha}\left(q^{+}, \boldsymbol{w}\right)\right\rangle_{q g g}^{r e g}, 1 & =-\frac{g^{2} q^{+}}{2(2 \pi)^{4}} \int_{\boldsymbol{x}, \boldsymbol{z}, \boldsymbol{z}^{\prime}} \int_{0}^{1} d \theta \int_{0}^{\theta} d \xi \frac{\sqrt{\xi}(1-\xi)}{\sqrt{\theta-\xi}} \tau_{\lambda_{1} \lambda_{2}}^{j m}(\theta, \xi) \phi_{\lambda_{2} \lambda}^{i l}(\xi) \frac{\boldsymbol{Y}^{l}\left(\boldsymbol{X}^{\prime}\right)^{m}}{\left(\boldsymbol{X}^{\prime}\right)^{2}} \\
& \times\left[\frac{V^{\delta \gamma}(\boldsymbol{x}) U^{d b}\left(\boldsymbol{z}^{\prime}\right) U^{c a}(\boldsymbol{z}) t_{\gamma \beta}^{b} t_{\beta \alpha}^{a}-t_{\delta \gamma}^{d} t_{\gamma \beta}^{c} V^{\beta \alpha}(\boldsymbol{w})}{(1-\theta)(\theta-\xi)\left(\boldsymbol{X}^{\prime}\right)^{2}+\xi(1-\xi)^{2} \boldsymbol{Y}^{2}}-\left(\boldsymbol{x}, \boldsymbol{z}^{\prime} \rightarrow \boldsymbol{y}\right)\right] \\
& \times \delta^{(2)}(\boldsymbol{w}-\boldsymbol{c})\left|q_{\lambda_{1}}^{\delta}\left((1-\theta) q^{+}, \boldsymbol{x}\right) g_{i}^{c}\left(\xi q^{+}, \boldsymbol{z}\right) g_{j}^{d}\left((\theta-\xi) q^{+}, \boldsymbol{z}^{\prime}\right)\right\rangle,
\end{aligned}
$$


where $\boldsymbol{y}$ denotes the transverse position of the intermediate quark and $\boldsymbol{c}$ is the center of energy of the three final partons (and coincides with the position $\boldsymbol{w}$ of the original quark):

$$
\boldsymbol{y} \equiv \frac{(1-\theta) \boldsymbol{x}+(\theta-\xi) \boldsymbol{z}^{\prime}}{1-\xi}, \quad \boldsymbol{c} \equiv(1-\xi) \boldsymbol{y}+\xi \boldsymbol{z}=(1-\theta) \boldsymbol{x}+\xi \boldsymbol{z}+(\theta-\xi) \boldsymbol{z}^{\prime} .
$$

Furthermore,

$$
\boldsymbol{Y} \equiv \boldsymbol{y}-\boldsymbol{z}, \quad \boldsymbol{X}^{\prime} \equiv \boldsymbol{x}-\boldsymbol{z}^{\prime},
$$

are the transverse separations between the daughter partons after each emission vertex. The spinorial structure is encoded in $\phi_{\lambda_{1} \lambda}^{i l}(\xi)$ (cf. eq. (3.7)) for the first emission vertex and respectively

$$
\tau_{\lambda_{1} \lambda}^{i j}(\theta, \xi) \equiv \chi_{\lambda_{1}}^{\dagger}\left[(2-\xi-\theta) \delta^{i j}+i(\xi-\theta) \varepsilon^{i j} \sigma^{3}\right] \chi_{\lambda},
$$

for the second vertex; notice the relation $\tau_{\lambda_{1} \lambda}^{i j}(\theta, 0)=\phi_{\lambda_{1} \lambda}^{i j}(\theta)$.

The structure of eq. (3.15) is reminiscent of that in eq. (3.10). The first term within the squared brackets represents the contribution of the final-state interaction, cf. figure 6.a, minus a piece generated by the initial-state interaction, cf. figure 6.b. The second term, as obtained from the first term via the replacements $\boldsymbol{x}, \boldsymbol{z}^{\prime} \rightarrow \boldsymbol{y}$, refers to the intermediate-state interaction, cf. figure 6.c, minus the remaining contribution of figure 6.b.

The contribution of the corresponding instantaneous graph, for which there is no analog of figure 6.c, is found as

$$
\begin{aligned}
& \left|q_{\lambda}^{\alpha}\left(q^{+}, \boldsymbol{w}\right)\right\rangle_{q g g}^{i n s t, 1}=-\frac{g^{2} q^{+}}{2(2 \pi)^{4}} \int_{\boldsymbol{x}, \boldsymbol{z}, \boldsymbol{z}^{\prime}} \int_{0}^{1} d \theta \int_{0}^{\theta} d \xi(1-\theta) \sqrt{\xi(\theta-\xi)} \chi_{\lambda_{1}}^{\dagger} \sigma_{j} \sigma_{i} \chi_{\lambda} \\
& \quad \times \frac{V^{\delta \gamma}(\boldsymbol{x}) U^{d b}\left(\boldsymbol{z}^{\prime}\right) U^{c a}(\boldsymbol{z}) t_{\gamma \beta}^{b} t_{\beta \alpha}^{a}-t_{\delta \gamma}^{d} t_{\gamma \beta}^{c} V^{\beta \alpha}(\boldsymbol{w})}{(1-\theta)(\theta-\xi)\left(\boldsymbol{X}^{\prime}\right)^{2}+\xi(1-\xi)^{2} \boldsymbol{Y}^{2}} \\
& \quad \times \delta^{(2)}(\boldsymbol{w}-\boldsymbol{c})\left|q_{\lambda_{1}}^{\delta}\left((1-\theta) q^{+}, \boldsymbol{x}\right) g_{i}^{c}\left(\xi q^{+}, \boldsymbol{z}\right) g_{j}^{d}\left((\theta-\xi) q^{+}, \boldsymbol{z}^{\prime}\right)\right\rangle,
\end{aligned}
$$

As in the previous subsection, it is convenient to group together "regular" and "instantaneous" contributions in a unique expression involved a non-local effective vertex - here, for the splitting of the original quark into a qgg system. Then the sum of eq. (3.15) and eq. (3.19) reads

$$
\begin{aligned}
& \left|q_{\lambda}^{\alpha}\left(q^{+}, \boldsymbol{w}\right)\right\rangle_{q g g}^{1}=-\frac{g^{2} q^{+}}{2(2 \pi)^{4}} \int_{\boldsymbol{x}, \boldsymbol{z}, \boldsymbol{z}^{\prime}} \int_{0}^{1} d \theta \int_{0}^{\theta} d \xi \frac{\boldsymbol{Y}^{m}\left(\boldsymbol{X}^{\prime}\right)^{n}}{\left(\boldsymbol{X}^{\prime}\right)^{2}} \\
& \quad \times\left[\Xi_{\lambda_{1} \lambda}^{i j m n} \frac{V^{\delta \gamma}(\boldsymbol{x}) U^{d b}\left(\boldsymbol{z}^{\prime}\right) U^{c a}(\boldsymbol{z}) t_{\gamma \beta}^{b} t_{\beta \alpha}^{a}-t_{\delta \gamma}^{d} t_{\gamma \beta}^{c} V^{\beta \alpha}(\boldsymbol{w})}{(1-\theta)(\theta-\xi)\left(\boldsymbol{X}^{\prime}\right)^{2}+\xi(1-\xi)^{2} \boldsymbol{Y}^{2}}-\left(\boldsymbol{x}, \boldsymbol{z}^{\prime} \rightarrow \boldsymbol{y}\right)\right] \\
& \quad \times \delta^{(2)}(\boldsymbol{w}-\boldsymbol{c})\left|q_{\lambda_{1}}^{\delta}\left((1-\theta) q^{+}, \boldsymbol{x}\right) g_{i}^{c}\left(\xi q^{+}, \boldsymbol{z}\right) g_{j}^{d}\left((\theta-\xi) q^{+}, \boldsymbol{z}^{\prime}\right)\right\rangle,
\end{aligned}
$$

with the effective vertex $\Xi_{\lambda_{1} \lambda}^{i j m n} \equiv \Xi_{\lambda_{1} \lambda}^{i j m n}\left(\boldsymbol{x}, \boldsymbol{z}, \boldsymbol{z}^{\prime}, \theta, \xi\right)$ defined as

$\Xi_{\lambda_{1} \lambda}^{i j m n}\left(\boldsymbol{x}, \boldsymbol{z}, \boldsymbol{z}^{\prime}, \theta, \xi\right) \equiv \sqrt{\xi(\theta-\xi)}\left(\frac{1-\xi}{\theta-\xi} \tau_{\lambda_{1} \lambda_{2}}^{j n}(\theta, \xi) \phi_{\lambda_{2} \lambda}^{i m}(\xi)-\delta^{m n}(1-\theta)\left(\chi_{\lambda_{1}}^{\dagger} \sigma_{j} \sigma_{i} \chi_{\lambda}\right) \frac{\left(\boldsymbol{X}^{\prime}\right)^{2}}{\boldsymbol{Y} \cdot \boldsymbol{X}^{\prime}}\right)$.

It is understood that the second piece in eq. (3.21) vanishes when $\boldsymbol{X}^{\prime}=\boldsymbol{x}-\boldsymbol{z}^{\prime} \rightarrow 0$. 


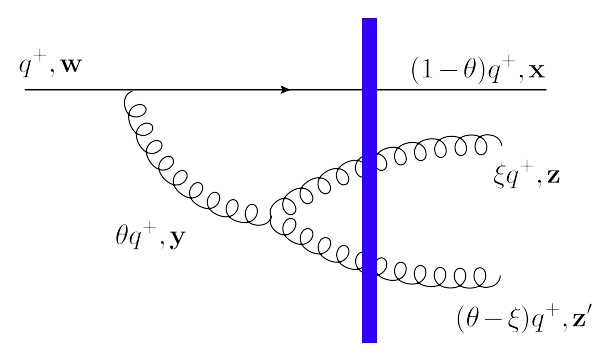

(a)

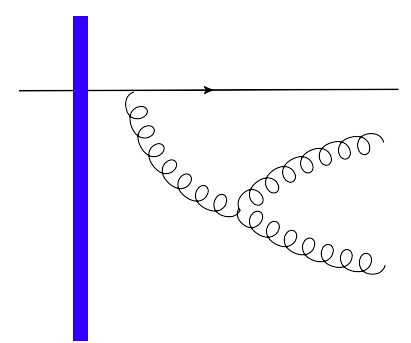

(b)

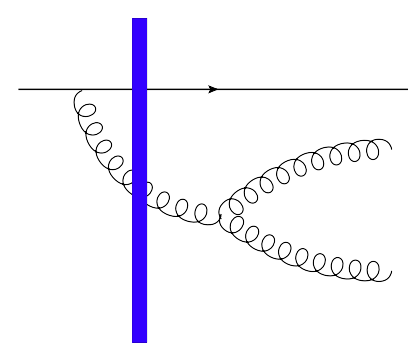

(c)

Figure 7. The three possible shockwave insertions for a final partonic state built with one quark and two gluons: (a) final-state scattering, (b) initial-state scattering, and (c) intermediate-state scattering.

The remaining topology for a final state with two gluons and a quark is very similar to that previously discussed for the tri-quark final state (compare the graphs in figures 7 and 3 , respectively). Hence, we shall simply present here the final result - the analog of eq. (3.12) — without further explanations. This reads

$$
\begin{aligned}
& \left|q_{\lambda}^{\alpha}\left(q^{+}, \boldsymbol{w}\right)\right\rangle_{q g g}^{2}=\frac{i g^{2} q^{+}}{2(2 \pi)^{4}} \int_{\boldsymbol{x}, \boldsymbol{z}, \boldsymbol{z}^{\prime}} \int_{0}^{1} d \theta \int_{0}^{\theta} d \xi \frac{\boldsymbol{R}^{m} \boldsymbol{Z}^{n}}{\boldsymbol{Z}^{2}} \\
& \quad \times\left[\Pi_{\lambda_{1} \lambda}^{i j m n} \frac{f^{a b e} V^{\gamma \beta}(\boldsymbol{x}) U^{d e}\left(\boldsymbol{z}^{\prime}\right) U^{c b}(\boldsymbol{z}) t_{\beta \alpha}^{a}-f^{a c d} t_{\gamma \beta}^{a} V^{\beta \alpha}(\boldsymbol{w})}{\theta^{2}(1-\theta) \boldsymbol{R}^{2}+\xi(\theta-\xi) \boldsymbol{Z}^{2}}-\left(\boldsymbol{z}, \boldsymbol{z}^{\prime} \rightarrow \boldsymbol{y}\right)\right] \\
& \quad \times \delta^{(2)}(\boldsymbol{w}-\boldsymbol{c})\left|q_{\lambda_{1}}^{\gamma}\left((1-\theta) q^{+}, \boldsymbol{x}\right) g_{i}^{c}\left(\xi q^{+}, \boldsymbol{z}\right) g_{j}^{d}\left((\theta-\xi) q^{+}, \boldsymbol{z}^{\prime}\right)\right\rangle,
\end{aligned}
$$

where we recall that $\boldsymbol{Z}=\boldsymbol{z}-\boldsymbol{z}^{\prime}, \boldsymbol{R}=\boldsymbol{x}-\boldsymbol{y}$, and $\boldsymbol{c}=(1-\theta) \boldsymbol{x}+\theta \boldsymbol{y}$, with $\boldsymbol{y}$ defined in eqs. (3.5).

The effective vertex $\Pi_{\lambda_{1} \lambda}^{i j m n} \equiv \Pi_{\lambda_{1} \lambda}^{i j m n}\left(\boldsymbol{x}, \boldsymbol{z}, \boldsymbol{z}^{\prime}, \theta, \xi\right)$ is defined as

$\Pi_{\lambda_{1} \lambda}^{i j m n}\left(\boldsymbol{x}, \boldsymbol{z}, \boldsymbol{z}^{\prime}, \theta, \xi\right) \equiv \sqrt{\xi(\theta-\xi)}(1-\theta)\left(\theta \Gamma^{n l i j}(\theta, \xi) \phi_{\lambda_{1} \lambda}^{l m}(\theta)-\delta^{i j} \delta^{m n} \delta_{\lambda_{1}} \frac{\theta-2 \xi}{\theta} \frac{\boldsymbol{Z}^{2}}{\boldsymbol{R} \cdot \boldsymbol{Z}}\right)$,

where the first term in the r.h.s. is the three-gluon vertex

$$
\Gamma^{n l i j}(\theta, \xi) \equiv \frac{1}{\theta} \delta_{n l} \delta_{i j}-\frac{1}{\xi} \delta_{n i} \delta_{l j}-\frac{1}{\theta-\xi} \delta_{n j} \delta_{l i},
$$

whereas the second term comes from the graphs with an instantaneous intermediate gluon.

To obtain an explicit expression for the subtracted term denoted as $\left(\boldsymbol{z}, \boldsymbol{z}^{\prime} \rightarrow \boldsymbol{y}\right)$ in eq. (3.22), the following identity is useful:

$$
f^{a b c} U^{d c}(\boldsymbol{y}) U^{e b}(\boldsymbol{y})=f^{b d e} U^{b a}(\boldsymbol{y}) .
$$

As before, one can check that, thanks to this subtraction, the two terms within the square brackets cancel each other in the limit $\boldsymbol{Z} \rightarrow 0$. 


\subsection{The trijet cross-section}

The leading-order cross-section for the inclusive three partons ("trijet") production in the quark-nucleus collision is computed similarly to eq. (2.9), that is, as the expectation value of the product of three number-density operators (themselves built with Fock space operators for bare partons).

\subsubsection{The $q q \bar{q}$ final state}

For the $q q \bar{q}$ final state, one writes (a factor $2 \pi \delta\left(k_{1}^{+}+k_{2}^{+}+k_{3}^{+}-q^{+}\right.$) is implicit in the l.h.s.)

$$
\begin{aligned}
& \frac{d \sigma^{q A \rightarrow q q \bar{q}+X}}{d^{3} k_{1} d^{3} k_{2} d^{3} k_{3}} \equiv \frac{1}{2 N_{c}}{ }^{\text {out }}\left\langle q_{\lambda}^{\alpha}\left(q^{+}, \boldsymbol{q}=\mathbf{0}\right)\left|\hat{\mathcal{N}}_{q}\left(k_{1}\right) \hat{\mathcal{N}}_{q}\left(k_{2}\right) \hat{\mathcal{N}}_{\bar{q}}\left(k_{3}\right)\right| q_{\lambda}^{\alpha}\left(q^{+}, \boldsymbol{q}=\mathbf{0}\right)\right\rangle^{\text {out }} \\
& =\frac{1}{2 N_{c}} \int_{\boldsymbol{w}, \overline{\boldsymbol{w}}} \quad \text { out } q q \bar{q}\left\langle q_{\lambda}^{\alpha}\left(q^{+}, \overline{\boldsymbol{w}}\right)\left|\hat{\mathcal{N}}_{q}\left(k_{1}\right) \hat{\mathcal{N}}_{q}\left(k_{2}\right) \hat{\mathcal{N}}_{\bar{q}}\left(k_{3}\right)\right| q_{\lambda}^{\alpha}\left(q^{+}, \boldsymbol{w}\right)\right\rangle_{q q \bar{q}}^{\text {out }},
\end{aligned}
$$

where as shown in the second line, only the $q q \bar{q}$ Fock-state component of the outgoing quark state, cf. eq. (3.12), is involved in this calculation. A priori there are four possible contractions for the product $\hat{\mathcal{N}}_{q}\left(k_{1}\right) \hat{\mathcal{N}}_{q}\left(k_{2}\right)$ of quark number density operators. However, two of these contractions correspond to non-planar graphs - these are graphs where the original parton on one side of the cut is contracted with the quark generated by the gluon decay on the other side of the cut (see figure 13.b in [1]) — which are suppressed in the multicolour limit $N_{c} \rightarrow \infty$. In what follows we shall systematically work in this limit, since it allows to simplify the colour structure of our results and also to render them physically transparent. Hence we shall keep only two of the four possible contractions those corresponding to planar graphs as illustrated in figure 8. We shall explicitly write the result for the case where the leading quark has momentum $k_{1}$ and the other quark has momentum $k_{2}$. The other term can be simply obtained by permuting these two momenta.

A straightforward calculation using the outgoing state in eq. (3.12) together with the Fock space rules summarised in appendix A yields ${ }^{4}$

$$
\begin{aligned}
& \frac{d \sigma^{q A \rightarrow q q \bar{q}+X}}{d k_{1}^{+} d^{2} \boldsymbol{k}_{1} d k_{2}^{+} d^{2} \boldsymbol{k}_{2} d k_{3}^{+} d^{2} \boldsymbol{k}_{3}}=\frac{\alpha_{s}^{2} C_{F} N_{f}}{2(2 \pi)^{10}\left(q^{+}\right)^{2}} \delta\left(q^{+}-k_{1}^{+}-k_{2}^{+}-k_{3}^{+}\right) \\
& \times \int_{\overline{\boldsymbol{x}}, \overline{\boldsymbol{z}}, \overline{\boldsymbol{z}}^{\prime}, \boldsymbol{x}, \boldsymbol{z}, \boldsymbol{z}^{\prime}} e^{-i \boldsymbol{k}_{1} \cdot(\boldsymbol{x}-\overline{\boldsymbol{x}})-i \boldsymbol{k}_{2} \cdot(\boldsymbol{z}-\overline{\boldsymbol{z}})-i k_{3} \cdot\left(\boldsymbol{z}^{\prime}-\overline{\boldsymbol{z}}^{\prime}\right)} \frac{\boldsymbol{R}^{i} \boldsymbol{Z}^{j} \overline{\boldsymbol{R}}^{m} \overline{\boldsymbol{Z}}^{n}}{\boldsymbol{Z}^{2} \overline{\boldsymbol{Z}}^{2}} \\
& \times\left[\mathcal{K}_{0}^{i j m n}\left(\boldsymbol{x}, \boldsymbol{z}, \boldsymbol{z}^{\prime}, \overline{\boldsymbol{x}}, \overline{\boldsymbol{z}}, \overline{\boldsymbol{z}}^{\prime}, \theta, \xi\right) \mathcal{W}_{0}\left(\boldsymbol{x}, \boldsymbol{z}, \boldsymbol{z}^{\prime}, \overline{\boldsymbol{x}}, \overline{\boldsymbol{z}}, \overline{\boldsymbol{z}}^{\prime}\right)\right. \\
& \left.-\left(\boldsymbol{z}, \boldsymbol{z}^{\prime} \rightarrow \boldsymbol{y}\right)-\left(\overline{\boldsymbol{z}}, \overline{\boldsymbol{z}}^{\prime} \rightarrow \overline{\boldsymbol{y}}\right)+\left(\boldsymbol{z}, \boldsymbol{z}^{\prime} \rightarrow \boldsymbol{y} \& \overline{\boldsymbol{z}}, \overline{\boldsymbol{z}}^{\prime} \rightarrow \overline{\boldsymbol{y}}\right)\right] \\
& +\left(k_{1}^{+} \leftrightarrow k_{2}^{+}, \boldsymbol{k}_{1} \leftrightarrow \boldsymbol{k}_{2}\right) .
\end{aligned}
$$

The notations here are similar to those in eq. (3.12). The transverse coordinates $\boldsymbol{x}, \boldsymbol{z}, \boldsymbol{z}^{\prime}, \boldsymbol{y}, \boldsymbol{w}$ refer to the direct amplitude (DA) and have the same meaning as in figure 3 and eq. (3.5). We use a bar to indicate the corresponding coordinates in the complex

\footnotetext{
${ }^{4}$ In the large- $N_{c}$ limit under consideration, the quark Casimir factor apparent in eq. (3.27) can be as well approximated as $C_{F} \simeq N_{c} / 2$; this applies to all the results involving $C_{F}$ to be shown in this paper.
} 
conjugate amplitude (CCA). We use the notations in eq. (3.6) for the transverse separations between the emitted partons, that is,

$$
\boldsymbol{R} \equiv \boldsymbol{x}-\boldsymbol{y}, \quad \boldsymbol{Z} \equiv \boldsymbol{z}-\boldsymbol{z}^{\prime}, \quad \overline{\boldsymbol{R}} \equiv \overline{\boldsymbol{x}}-\overline{\boldsymbol{y}}, \quad \overline{\boldsymbol{Z}} \equiv \overline{\boldsymbol{z}}-\overline{\boldsymbol{z}}^{\prime}
$$

The longitudinal momentum fractions $\theta$ and $\xi$ are the same in the DA and in the CCA, since they are fully fixed by the kinematics of the final state, as follows:

$$
\theta=1-\frac{k_{1}^{+}}{q^{+}}, \quad \xi=\frac{k_{2}^{+}}{q^{+}}
$$

The $\delta$-function enforcing longitudinal momentum conservation implies $k_{3}^{+}=(\theta-\xi) q^{+}$, which in particular requires $\theta \geq \xi$ (i.e. $k_{1}^{+}+k_{2}^{+} \leq q^{+}$).

Let us now explain the new structures occurring in eq. (3.27). The tensorial kernel is defined as

$$
\mathcal{K}_{0}^{i j m n}\left(\boldsymbol{x}, \boldsymbol{z}, \boldsymbol{z}^{\prime}, \overline{\boldsymbol{x}}, \overline{\boldsymbol{z}}, \overline{\boldsymbol{z}}^{\prime}, \theta, \xi\right) \equiv \frac{\Phi_{\lambda_{3} \lambda_{2} \lambda_{1} \lambda}^{i j}\left(\boldsymbol{x}, \boldsymbol{z}, \boldsymbol{z}^{\prime}, \theta, \xi\right) \Phi_{\lambda_{3} \lambda_{2} \lambda_{1} \lambda}^{m n *}\left(\overline{\boldsymbol{x}}, \overline{\boldsymbol{z}}, \overline{\boldsymbol{z}}^{\prime}, \theta, \xi\right)}{\left[\theta^{2}(1-\theta) \boldsymbol{R}^{2}+\xi(\theta-\xi) \boldsymbol{Z}^{2}\right]\left[\theta^{2}(1-\theta) \overline{\boldsymbol{R}}^{2}+\xi(\theta-\xi) \overline{\boldsymbol{Z}}^{2}\right]},
$$

with the effective vertex $\Phi_{\lambda_{3} \lambda_{2} \lambda_{1} \lambda}^{i j}$ introduced in eq. (3.13) (the star in $\Phi^{*}$ denotes complex conjugation). The product of effective vertices in the numerator can be explicitly computed as

$$
\begin{aligned}
& \left.\Phi_{\lambda_{3} \lambda_{2} \lambda_{1} \lambda}^{i j} \Phi_{\lambda_{3} \lambda_{2} \lambda_{1} \lambda}^{m n *}\right|_{\text {non-inst. }} \\
& =4(1-\theta)^{2}\left[(\theta-2 \xi)^{2} \delta^{r j} \delta^{t n}+\theta^{2}\left(\delta^{r t} \delta^{j n}-\delta^{r n} \delta^{j t}\right)\right]\left[(2-\theta)^{2} \delta^{r i} \delta^{t m}+\theta^{2}\left(\delta^{r t} \delta^{i m}-\delta^{r m} \delta^{i t}\right)\right] .
\end{aligned}
$$

As emphasised by our notation, in evaluating this product we have excluded the instantaneous pieces from the two effective vertices. These pieces have a very simple tensorial structure, so they can be easily inserted when needed. ${ }^{5}$

The effects of the collision are encoded in the function $\mathcal{W}_{0}$, defined as the following linear combination of partonic $S$-matrices:

$$
\begin{aligned}
& \mathcal{W}_{0}\left(\boldsymbol{x}, \boldsymbol{z}, \boldsymbol{z}^{\prime}, \overline{\boldsymbol{x}}, \overline{\boldsymbol{z}}, \overline{\boldsymbol{z}}^{\prime}\right) \\
& \equiv S_{q q \bar{q} \bar{q} q}\left(\boldsymbol{x}, \boldsymbol{z}, \boldsymbol{z}^{\prime}, \overline{\boldsymbol{x}}, \overline{\boldsymbol{z}}, \overline{\boldsymbol{z}}^{\prime}\right)-S_{q q \bar{q} \bar{q}}\left(\boldsymbol{x}, \boldsymbol{z}, \boldsymbol{z}^{\prime}, \overline{\boldsymbol{w}}\right)-S_{q \bar{q} \bar{q} q}\left(\boldsymbol{w}, \overline{\boldsymbol{x}}, \overline{\boldsymbol{z}}, \overline{\boldsymbol{z}}^{\prime}\right)+\mathcal{S}(\boldsymbol{w}, \overline{\boldsymbol{w}})
\end{aligned}
$$

Our notations for the partonic $S$-matrices are intended to describe (via the lower scripts) the partonic composition of the multi-parton system which scatters off the shockwave and to also distinguish (via a bar on the transverse coordinates) between partons in the DA and in the CCA, respectively. The ordering of the transverse coordinates in the argument follows that of the lower subscripts. The indices/arguments corresponding to partons in the DA appear on the left to those representing the CCA. When reading the lower indices,

\footnotetext{
${ }^{5}$ These instantaneous pieces do not contribute to either the soft, or the collinear, limit that we shall study later; so, a result like eq. (3.31) is indeed sufficient for our purposes in this paper.
} 


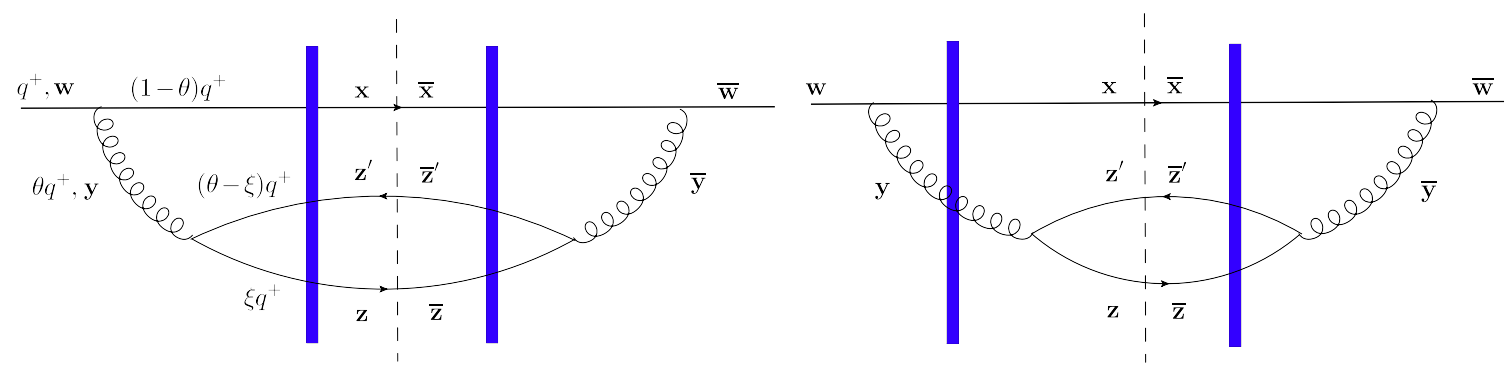

Figure 8. Left: the graph corresponding to final-state interactions in both the DA and the CCA; its colour structure is encoded in the 6 -quark $S$-matrix shown in eq. (3.33). Right: a graph describing interference between interactions in the intermediate state in the DA and respectively in the final state in the CCA; the associated $S$-matrix is obtained by replacing $\boldsymbol{z} \rightarrow \boldsymbol{y}$ and $\boldsymbol{z}^{\prime} \rightarrow \boldsymbol{y}$ in eq. (3.33).

one should also keep in mind that a quark in the CCA formally counts like an antiquark (from the viewpoint of the direction it its colour charge flow).

According to these conventions, the $S$-matrix $S_{q q \bar{q} q \bar{q} q}\left(\boldsymbol{x}, \boldsymbol{z}, \boldsymbol{z}^{\prime}, \overline{\boldsymbol{x}}, \overline{\boldsymbol{z}}, \overline{\boldsymbol{z}}^{\prime}\right)$ refers to the eikonal scattering of a system made with 6 quarks: 2 quarks $(\boldsymbol{x}, \boldsymbol{z})$ and one anti-quark $\left(\boldsymbol{z}^{\prime}\right)$ in the DA, together with 2 "anti-quarks" $(\overline{\boldsymbol{x}}, \overline{\boldsymbol{z}})$ and one "quark" $\left(\overline{\boldsymbol{z}}^{\prime}\right)$ in the CCA. This describes the situation where the collision occurs in the final state (i.e. after the second splitting) in both the DA and in the CCA (see figure 8.left), and reads

$$
\begin{aligned}
S_{q q \bar{q} \bar{q} q}\left(\boldsymbol{x}, \boldsymbol{z}, \boldsymbol{z}^{\prime}, \overline{\boldsymbol{x}}, \overline{\boldsymbol{z}}, \overline{\boldsymbol{z}}^{\prime}\right) & \equiv \frac{2}{C_{F} N_{c}}\left\langle\operatorname{tr}\left(V^{\dagger}(\overline{\boldsymbol{x}}) V(\boldsymbol{x}) t^{a} t^{b}\right) \operatorname{tr}\left(V\left(\overline{\boldsymbol{z}}^{\prime}\right) t^{b} V^{\dagger}(\overline{\boldsymbol{z}}) V(\boldsymbol{z}) t^{a} V^{\dagger}\left(\boldsymbol{z}^{\prime}\right)\right)\right\rangle, \\
& \simeq \mathcal{Q}\left(\boldsymbol{x}, \boldsymbol{z}^{\prime}, \overline{\boldsymbol{z}}^{\prime}, \overline{\boldsymbol{x}}\right) \mathcal{S}(\boldsymbol{z}, \overline{\boldsymbol{z}}),
\end{aligned}
$$

where the approximate equality holds for large $N_{c}$ : in this limit, the 6-quark $S$-matrix factorises into the product of a dipole, $\mathcal{S}(\boldsymbol{z}, \overline{\boldsymbol{z}})$, and a quadrupole, $\mathcal{Q}\left(\boldsymbol{x}, \boldsymbol{z}^{\prime}, \overline{\boldsymbol{z}}^{\prime}, \overline{\boldsymbol{x}}\right)$.

Furthermore, $S_{q q \bar{q} \bar{q}}\left(\boldsymbol{x}, \boldsymbol{z}, \boldsymbol{z}^{\prime}, \overline{\boldsymbol{w}}\right)$ and $S_{q \bar{q} \bar{q} q}\left(\boldsymbol{w}, \overline{\boldsymbol{x}}, \overline{\boldsymbol{z}}, \overline{\boldsymbol{z}}^{\prime}\right)$ represent interference terms where the collision with the shockwave occurs in the final state on one side of the cut, and in the initial state (prior to the first splitting) on the other side; e.g.,

$$
S_{q q \bar{q} \bar{q}}\left(\boldsymbol{x}, \boldsymbol{z}, \boldsymbol{z}^{\prime}, \overline{\boldsymbol{w}}\right)=\frac{2}{C_{F} N_{c}}\left\langle\operatorname{tr}\left[V(\boldsymbol{x}) t^{a} V^{\dagger}(\overline{\boldsymbol{w}}) t^{b}\right] \operatorname{tr}\left[V(\boldsymbol{z}) t^{a} V^{\dagger}\left(\boldsymbol{z}^{\prime}\right) t^{b}\right]\right\rangle \simeq \mathcal{S}(\boldsymbol{z}, \overline{\boldsymbol{w}}) \mathcal{S}\left(\boldsymbol{x}, \boldsymbol{z}^{\prime}\right) .
$$

Finally, the colour dipole $\mathcal{S}(\boldsymbol{w}, \overline{\boldsymbol{w}}) \equiv S_{q \bar{q}}(\boldsymbol{w}, \overline{\boldsymbol{w}})$ describes initial-state interactions in both the DA and the CCA.

The normalisation factors in the above definitions are such that all the individual $S$-matrices reduce to unity in the absence of scattering. Accordingly, both the linear combination in eq. (3.32) and the cross-section (3.27) vanish in that limit, as expected. The overall colour structure becomes remarkably simple at large $N_{c}$ :

$\mathcal{W}_{0}\left(\boldsymbol{x}, \boldsymbol{z}, \boldsymbol{z}^{\prime}, \overline{\boldsymbol{x}}, \overline{\boldsymbol{z}}, \overline{\boldsymbol{z}}^{\prime}\right) \simeq \mathcal{Q}\left(\boldsymbol{x}, \boldsymbol{z}^{\prime}, \overline{\boldsymbol{z}}^{\prime}, \overline{\boldsymbol{x}}\right) \mathcal{S}(\boldsymbol{z}, \overline{\boldsymbol{z}})-\mathcal{S}(\boldsymbol{z}, \overline{\boldsymbol{w}}) \mathcal{S}\left(\boldsymbol{x}, \boldsymbol{z}^{\prime}\right)-\mathcal{S}(\boldsymbol{w}, \overline{\boldsymbol{z}}) \mathcal{S}\left(\overline{\boldsymbol{z}}^{\prime}, \overline{\boldsymbol{x}}\right)+\mathcal{S}(\boldsymbol{w}, \overline{\boldsymbol{w}})$

The three other terms within the square brackets in eq. (3.27), which are obtained as various limits of the first term, refer to situations where the scattering in the final 
state gets replaced by scattering in the intermediate state, as explained in relation with eq. (3.10). E.g. the second term within the square brackets, denoted as $\left(\boldsymbol{z}, \boldsymbol{z}^{\prime} \rightarrow \boldsymbol{y}\right)$, is illustrated in figure 8.right. We recall that this term can be obtained by letting $\boldsymbol{Z} \rightarrow 0$ in the kernel (3.30) (which in particular means using the simplified version of the effective vertex shown in eq. (3.14)) and replacing $\boldsymbol{z} \rightarrow \boldsymbol{y}$ and $\boldsymbol{z}^{\prime} \rightarrow \boldsymbol{y}$ in the arguments of the partonic $S$-matrices in the r.h.s. of eq. (3.32).

It is quite instructive to display the version of eq. (3.32) which applies to the last term in eq. (3.27), as obtained by simultaneously replacing $\left(\boldsymbol{z}, \boldsymbol{z}^{\prime} \rightarrow \boldsymbol{y}\right)$ and $\left(\overline{\boldsymbol{z}}, \overline{\boldsymbol{z}}^{\prime} \rightarrow \overline{\boldsymbol{y}}\right)$. This describes the case where there are no interactions with the shockwave in the final $q q \bar{q}$ state, neither in the DA nor in the CCA. Working at large $N_{c}$ for definiteness, cf. eq. (3.35), one finds

$$
\mathcal{W}_{0}(\boldsymbol{x}, \boldsymbol{y}, \boldsymbol{y}, \overline{\boldsymbol{x}}, \overline{\boldsymbol{y}}, \overline{\boldsymbol{y}}) \simeq \mathcal{Q}(\boldsymbol{x}, \boldsymbol{y}, \overline{\boldsymbol{y}}, \overline{\boldsymbol{x}}) \mathcal{S}(\boldsymbol{y}, \overline{\boldsymbol{y}})-\mathcal{S}(\boldsymbol{y}, \overline{\boldsymbol{w}}) \mathcal{S}(\boldsymbol{x}, \boldsymbol{y})-\mathcal{S}(\boldsymbol{w}, \overline{\boldsymbol{y}}) \mathcal{S}(\overline{\boldsymbol{y}}, \overline{\boldsymbol{x}})+\mathcal{S}(\boldsymbol{w}, \overline{\boldsymbol{w}})
$$

This $S$-matrix structure is identical to that occurring in the integrand of eq. (2.17) for LO quark-gluon production $(q A \rightarrow q g+X)$. This is easy to understand: in both cases, the interactions with the nuclear shockwave refer to the partons involved in the branching $q \rightarrow q g$.

So far, we have not specified the $x_{g}$-argument of the various $S$-matrices in eq. (3.32), i.e. the "minus" longitudinal momentum fraction of the gluons from the nuclear target which are involved in the production of the three-parton state. Clearly, this is given by the generalisation of eq. (2.1) to a final state involving three partons, that is,

$$
x_{g}=\frac{\boldsymbol{k}_{1}^{2}}{x_{1} s}+\frac{\boldsymbol{k}_{2}^{2}}{x_{2} s}+\frac{\boldsymbol{k}_{3}^{2}}{x_{3} s},
$$

where $x_{i}=k_{i}^{+} / Q^{+}$are the "plus" longitudinal momentum fractions of the produced partons. Needless to say, this value for $x_{g}$ applies not only to the $q q \bar{q}$ final state discussed so far, but also to the $q g g$ final states to which we now turn.

\subsubsection{The $q g g$ final state}

For the $q g g$ final state, the trijet cross-section is computed as (once again, a factor $2 \pi \delta\left(k_{1}^{+}+\right.$ $k_{2}^{+}+k_{3}^{+}-q^{+}$) is implicitly understood in the l.h.s.)

$$
\begin{aligned}
\frac{d \sigma^{q A \rightarrow q g g+X}}{d^{3} k_{1} d^{3} k_{2} d^{3} k_{3}} & \equiv \frac{1}{2 N_{c}}{ }^{\text {out }}\left\langle q_{\lambda}^{\alpha}\left(q^{+}, \boldsymbol{q}=\mathbf{0}\right)\left|\hat{\mathcal{N}}_{q}\left(k_{1}\right) \hat{\mathcal{N}}_{g}\left(k_{2}\right) \hat{\mathcal{N}}_{g}\left(k_{3}\right)\right| q_{\lambda}^{\alpha}\left(q^{+}, \boldsymbol{q}=\mathbf{0}\right)\right\rangle^{\text {out }} \\
& =\frac{1}{2 N_{c}} \int_{\boldsymbol{w}, \overline{\boldsymbol{w}}} \stackrel{\text { out }}{q g g}\left\langle q_{\lambda}^{\alpha}\left(q^{+}, \overline{\boldsymbol{w}}\right)\left|\hat{\mathcal{N}}_{q}\left(k_{1}\right) \hat{\mathcal{N}}_{g}\left(k_{2}\right) \hat{\mathcal{N}}_{g}\left(k_{3}\right)\right| q_{\lambda}^{\alpha}\left(q^{+}, \boldsymbol{w}\right)\right\rangle_{q g g}^{\text {out }}
\end{aligned}
$$

The second line involves the $q g g$ Fock component of the quark outgoing state, which, as explained in section 3.1.2, is the sum of two contributions, corresponding to two different topologies for the gluon emission vertices, as illustrated in figure 6 and figure 7 , respectively. It is therefore natural to split the cross-section in eq. (3.38) into three contributions. In the first one, the topology in figure 6 is used for the quark LCWF in both the DA and the 


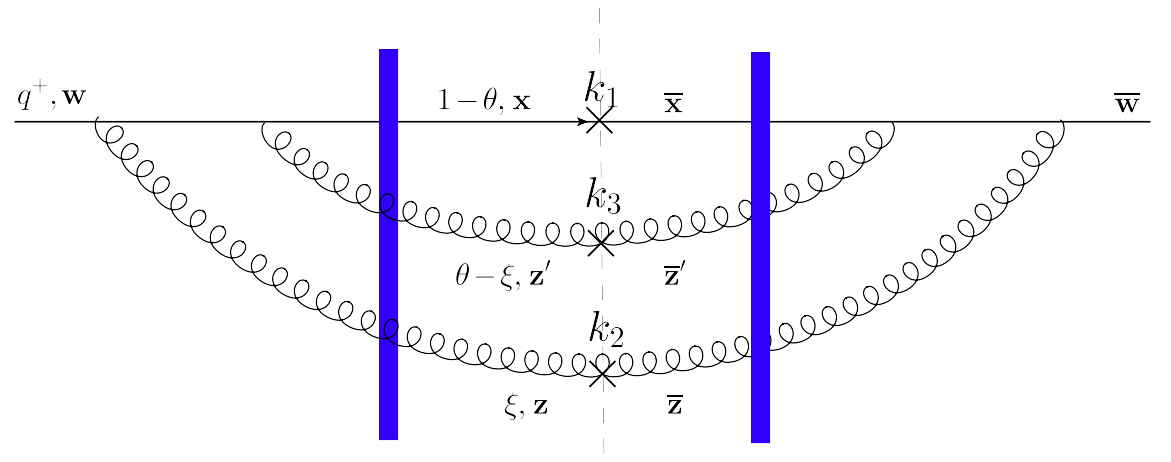

Figure 9. A contribution to the $q g g$ cross-section (3.39) which features final-state interactions in both the DA and the CCA; the respective colour structure is shown in eq. (3.43). There is a similar diagram in which the momenta $k_{2}$ and $k_{3}$ of the final gluons are exchanged with each other.

CCA. Similarly, the second contributions involves the topology in figure 7 alone. Finally, the third contribution represents interferences between the two topologies. Besides, each of these three contributions is built with two pieces, corresponding to the two possible permutations for the momentum labels of the produced gluons. As before, we shall work in the limit of a large number of colours, in which one discards the non-planar graphs. One should however pay attention to the fact that the symmetry of the triple gluon vertex makes that the graphs generated by exchanging the two final gluons in figure 7 are still planar, and therefore must be kept in the final result. This will result in appropriate symmetry factors.

A straightforward calculation using eq. (3.20) yields the following expression for the first piece of the cross-section:

$$
\begin{aligned}
& \frac{d \sigma_{(1)}^{q A \rightarrow q g g+X}}{d k_{1}^{+} d^{2} \boldsymbol{k}_{1} d k_{2}^{+} d^{2} \boldsymbol{k}_{2} d k_{3}^{+} d^{2} \boldsymbol{k}_{3}}=\frac{\alpha_{s}^{2} C_{F}^{2}}{2(2 \pi)^{10}\left(q^{+}\right)^{2}} \delta\left(q^{+}-k_{1}^{+}-k_{2}^{+}-k_{3}^{+}\right) \\
& \times \int_{\overline{\boldsymbol{x}}, \overline{\boldsymbol{z}}, \overline{\boldsymbol{z}}^{\prime}, \boldsymbol{x}, \boldsymbol{z}, \boldsymbol{z}^{\prime}} e^{-i \boldsymbol{k}_{1} \cdot(\boldsymbol{x}-\overline{\boldsymbol{x}})-i \boldsymbol{k}_{2} \cdot(\boldsymbol{z}-\overline{\boldsymbol{z}})-i k_{3} \cdot\left(\boldsymbol{z}^{\prime}-\overline{\boldsymbol{z}}^{\prime}\right)} \frac{\boldsymbol{Y}^{m}\left(\boldsymbol{X}^{\prime}\right)^{n} \overline{\boldsymbol{Y}}^{p}\left(\overline{\boldsymbol{X}}^{\prime}\right)^{q}}{\left(\boldsymbol{X}^{\prime}\right)^{2}\left(\overline{\boldsymbol{X}}^{\prime}\right)^{2}} \\
& \times\left[\mathcal{K}_{1}^{m n p q}\left(\boldsymbol{x}, \boldsymbol{z}, \boldsymbol{z}^{\prime}, \overline{\boldsymbol{x}}, \overline{\boldsymbol{z}}, \overline{\boldsymbol{z}}^{\prime}, \theta, \xi\right) \mathcal{W}_{1}\left(\boldsymbol{x}, \boldsymbol{z}, \boldsymbol{z}^{\prime}, \overline{\boldsymbol{x}}, \overline{\boldsymbol{z}}, \overline{\boldsymbol{z}}^{\prime}\right)\right. \\
& \left.-\left(\boldsymbol{x}, \boldsymbol{z}^{\prime} \rightarrow \boldsymbol{y}\right)-\left(\overline{\boldsymbol{x}}, \overline{\boldsymbol{z}}^{\prime} \rightarrow \overline{\boldsymbol{y}}\right)+\left(\boldsymbol{x}, \boldsymbol{z}^{\prime} \rightarrow \boldsymbol{y} \& \overline{\boldsymbol{x}}, \overline{\boldsymbol{z}}^{\prime} \rightarrow \overline{\boldsymbol{y}}\right)\right] \\
& +\left(k_{2}^{+} \leftrightarrow k_{3}^{+}, \boldsymbol{k}_{2} \leftrightarrow \boldsymbol{k}_{3}\right) .
\end{aligned}
$$

The notations for the transverse coordinates which appear in this equation are easily grasped by comparing with eq. (3.20). The new tensorial kernel $\mathcal{K}_{1}^{\text {mnpq }}$ is defined as

$$
\mathcal{K}_{1}^{m n p q} \equiv \frac{\Xi_{\lambda_{1} \lambda}^{i j m n}\left(\boldsymbol{x}, \boldsymbol{z}, \boldsymbol{z}^{\prime}, \theta, \xi\right) \Xi_{\lambda_{1} \lambda}^{i j p q *}\left(\overline{\boldsymbol{x}}, \overline{\boldsymbol{z}}, \overline{\boldsymbol{z}}^{\prime}, \theta, \xi\right)}{\left[(1-\theta)(\theta-\xi)\left(\boldsymbol{X}^{\prime}\right)^{2}+\xi(1-\xi)^{2} \boldsymbol{Y}^{2}\right]\left[(1-\theta)(\theta-\xi)\left(\overline{\boldsymbol{X}}^{\prime}\right)^{2}+\xi(1-\xi)^{2} \overline{\boldsymbol{Y}}^{2}\right]},
$$

with the effective vertex $\Xi_{\lambda_{1} \lambda}^{i j m n}$ introduced in eq. (3.21) and the longitudinal momentum fractions $\theta$ and $\xi$ shown in eq. (3.29). The product of effective vertices in the numerator 
can be explicitly computed:

$$
\begin{aligned}
\left.\Xi_{\lambda_{1} \lambda}^{i j m n} \Xi_{\lambda_{1} \lambda}^{i j p q *}\right|_{\text {non-inst. }} & =\frac{\xi(1-\xi)^{2}}{\theta-\xi} \tau_{\lambda_{1} \lambda_{2}}^{j n}(\theta, \xi) \tau_{\lambda_{1} \lambda_{3}}^{j q *}(\theta, \xi) \phi_{\lambda_{2} \lambda}^{i m}(\xi) \phi_{\lambda_{3} \lambda}^{i p, *}(\xi) \\
& =8 \delta^{m p} \delta^{n q} \frac{\xi(1-\xi)^{2}}{\theta-\xi}\left[1+(1-\xi)^{2}\right]\left[(1-\theta)^{2}+(1-\xi)^{2}\right]
\end{aligned}
$$

Furthermore, $\mathcal{W}_{1}$ denotes the following linear combination of partonic $S$-matrices:

$$
\begin{aligned}
& \mathcal{W}_{1}\left(\boldsymbol{x}, \boldsymbol{z}, \boldsymbol{z}^{\prime}, \overline{\boldsymbol{x}}, \overline{\boldsymbol{z}}, \overline{\boldsymbol{z}}^{\prime}\right) \\
& \equiv S_{q g q \bar{q} g g}^{(1)}\left(\boldsymbol{x}, \boldsymbol{z}, \boldsymbol{z}^{\prime}, \overline{\boldsymbol{x}}, \overline{\boldsymbol{z}}, \overline{\boldsymbol{z}}^{\prime}\right)-S_{q g g \bar{q}}^{(1)}\left(\boldsymbol{x}, \boldsymbol{z}, \boldsymbol{z}^{\prime}, \overline{\boldsymbol{w}}\right)-S_{q \bar{q} g g}^{(1)}\left(\boldsymbol{w}, \overline{\boldsymbol{x}}, \overline{\boldsymbol{z}}, \overline{\boldsymbol{z}}^{\prime}\right)+\mathcal{S}(\boldsymbol{w}, \overline{\boldsymbol{w}}) .
\end{aligned}
$$

We use the same notations as explained after eq. (3.32) in order to synthetically summarize the partonic content for both the DA and the CCA. The first $S$-matrix in the r.h.s., that is,

$$
\begin{aligned}
S_{q g g \bar{q} g g}^{(1)}\left(\boldsymbol{x}, \boldsymbol{z}, \boldsymbol{z}^{\prime}, \overline{\boldsymbol{x}}, \overline{\boldsymbol{z}}, \overline{\boldsymbol{z}}^{\prime}\right) & \equiv \frac{1}{C_{F}^{2} N_{c}}\left\langle\operatorname{tr}\left[V^{\dagger}(\overline{\boldsymbol{x}}) V(\boldsymbol{x}) t^{b} t^{a} t^{f} t^{e}\right]\left[U^{\dagger}(\overline{\boldsymbol{z}}) U(\boldsymbol{z})\right]^{f a}\left[U^{\dagger}\left(\overline{\boldsymbol{z}}^{\prime}\right) U\left(\boldsymbol{z}^{\prime}\right)\right]^{e b}\right\rangle \\
& \simeq \mathcal{Q}\left(\boldsymbol{x}, \boldsymbol{z}^{\prime}, \overline{\boldsymbol{z}}^{\prime}, \overline{\boldsymbol{x}}\right) \mathcal{Q}\left(\boldsymbol{z}^{\prime}, \boldsymbol{z}, \overline{\boldsymbol{z}}, \overline{\boldsymbol{z}}^{\prime}\right) \mathcal{S}(\boldsymbol{z}, \overline{\boldsymbol{z}}),
\end{aligned}
$$

describes final-state interactions in both the DA and the CCA and hence it includes Wilson lines for 6 partons: one quark $(\boldsymbol{x})$ and two gluons $\left(\boldsymbol{z}, \boldsymbol{z}^{\prime}\right)$ in the DA, and one "anti-quark" $(\overline{\boldsymbol{x}})$ and two gluons $\left(\overline{\boldsymbol{z}}, \overline{\boldsymbol{z}}^{\prime}\right)$ in the CCA (see figure 9 ). Similarly,

$$
\begin{aligned}
S_{q g g \bar{q}}^{(1)}\left(\boldsymbol{x}, \boldsymbol{z}, \boldsymbol{z}^{\prime}, \overline{\boldsymbol{w}}\right) & \equiv \frac{1}{C_{F}^{2} N_{c}}\left\langle\operatorname{tr}\left[V^{\dagger}(\overline{\boldsymbol{w}}) t^{c} t^{d} V(\boldsymbol{x}) t^{b} t^{a}\right] U^{d b}\left(\boldsymbol{z}^{\prime}\right) U^{c a}(\boldsymbol{z})\right\rangle \\
& \simeq \mathcal{S}\left(\boldsymbol{x}, \boldsymbol{z}^{\prime}\right) \mathcal{S}\left(\boldsymbol{z}^{\prime}, \boldsymbol{z}\right) \mathcal{S}(\boldsymbol{z}, \overline{\boldsymbol{w}}),
\end{aligned}
$$

is an interference term between final-state and initial-state interactions. Once again, the large $N_{c}$ version of $\mathcal{W}_{1}$ can be fully expressed in terms of colour dipoles and quadrupoles:

$$
\begin{aligned}
\mathcal{W}_{1}\left(\boldsymbol{x}, \boldsymbol{z}, \boldsymbol{z}^{\prime}, \overline{\boldsymbol{x}}, \overline{\boldsymbol{z}}, \overline{\boldsymbol{z}}^{\prime}\right) \simeq & \mathcal{Q}\left(\boldsymbol{x}, \boldsymbol{z}^{\prime}, \overline{\boldsymbol{z}}^{\prime}, \overline{\boldsymbol{x}}\right) \mathcal{Q}\left(\boldsymbol{z}^{\prime}, \boldsymbol{z}, \overline{\boldsymbol{z}}, \overline{\boldsymbol{z}}^{\prime}\right) \mathcal{S}(\boldsymbol{z}, \overline{\boldsymbol{z}})+\mathcal{S}(\boldsymbol{w}, \overline{\boldsymbol{w}})- \\
& -\mathcal{S}\left(\boldsymbol{x}, \boldsymbol{z}^{\prime}\right) \mathcal{S}\left(\boldsymbol{z}^{\prime}, \boldsymbol{z}\right) \mathcal{S}(\boldsymbol{z}, \overline{\boldsymbol{w}})-\mathcal{S}(\boldsymbol{w}, \overline{\boldsymbol{z}}) \mathcal{S}\left(\overline{\boldsymbol{z}}, \overline{\boldsymbol{z}}^{\prime}\right) \mathcal{S}\left(\overline{\boldsymbol{z}}^{\prime}, \overline{\boldsymbol{x}}\right)
\end{aligned}
$$

As a simple check, we notice that after the double replacement $\left(\boldsymbol{x}, \boldsymbol{z}^{\prime} \rightarrow \boldsymbol{y} \& \overline{\boldsymbol{x}}, \overline{\boldsymbol{z}}^{\prime} \rightarrow \overline{\boldsymbol{y}}\right)$ (which yields the last term within the square brackets in eq. (3.39)), the r.h.s. of eq. (3.42) takes the same form as for the scattering of a quark-gluon pair, ${ }^{6}$ as expected.

Consider also the second piece in the cross-section (3.39), as obtained by permuting the momenta $k_{2}$ and $k_{3}$ of the final gluons. This can be computed from the first piece (the one explicitly shown in eq. (3.39) and illustrated in figure 9) by exchanging $\boldsymbol{k}_{2} \leftrightarrow \boldsymbol{k}_{3}$ within the Fourier phases and $k_{2}^{+} \leftrightarrow k_{3}^{+}$within the longitudinal momentum fractions. Alternatively, and equivalently, this second term can be written as a Fourier transform which involves exactly the same Fourier phases as the first term, but with the following changes of variables in the remaining part of the integrand: $\boldsymbol{z} \leftrightarrow \boldsymbol{z}^{\prime}, \overline{\boldsymbol{z}} \leftrightarrow \overline{\boldsymbol{z}}^{\prime}$, and $\xi \leftrightarrow \theta-\xi$. This amounts to permuting the transverse coordinates and the longitudinal momentum fractions assigned to the final gluons in the amplitude in figure 6 (and similarly for the CCA).

\footnotetext{
${ }^{6}$ That is, it reduces to the $S$-matrix structure visible in the integrand of eq. (2.10), up to some relabelling of variables.
} 


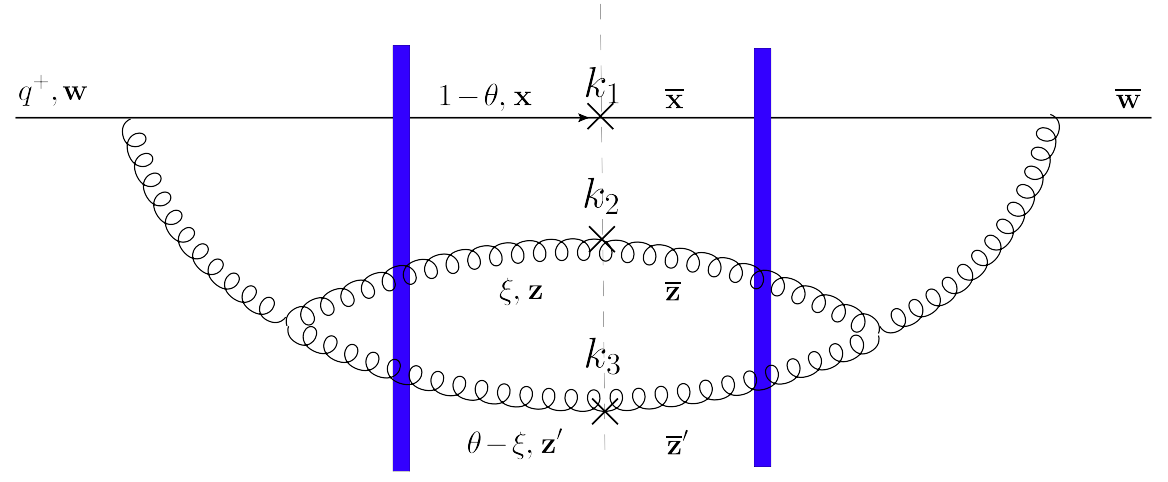

Figure 10. A graph contributing to the cross-section in (3.46); the associated $S$-matrix is shown in eq. (3.50).

We now turn to the second contribution to the cross-section for $q g g$ production, that involving the topology in figure 7. We now use eq. (3.22) to deduce

$$
\begin{gathered}
\frac{d \sigma_{(2)}^{q A \rightarrow q g g+X}}{d k_{1}^{+} d^{2} \boldsymbol{k}_{1} d k_{2}^{+} d^{2} \boldsymbol{k}_{2} d k_{3}^{+} d^{2} \boldsymbol{k}_{3}}=\frac{4 \alpha_{s}^{2} C_{F} N_{c}}{2(2 \pi)^{10}\left(q^{+}\right)^{2}} \delta\left(q^{+}-k_{1}^{+}-k_{2}^{+}-k_{3}^{+}\right) \\
\times \int_{\overline{\boldsymbol{x}}, \overline{\boldsymbol{z}}, \overline{\boldsymbol{z}}^{\prime}, \boldsymbol{x}, \boldsymbol{z}, \boldsymbol{z}^{\prime}} e^{-i \boldsymbol{k}_{1} \cdot(\boldsymbol{x}-\overline{\boldsymbol{x}})-i \boldsymbol{k}_{2} \cdot(\boldsymbol{z}-\overline{\boldsymbol{z}})-i \boldsymbol{k}_{3} \cdot\left(\boldsymbol{z}^{\prime}-\overline{\boldsymbol{z}}^{\prime}\right)} \frac{\boldsymbol{R}^{m} \boldsymbol{Z}^{n} \overline{\boldsymbol{R}}^{p} \overline{\boldsymbol{Z}}^{q}}{\boldsymbol{Z}^{2} \overline{\boldsymbol{Z}}^{2}} \\
\times\left[\mathcal{K}_{2}^{m n p q q}\left(\boldsymbol{x}, \boldsymbol{z}, \boldsymbol{z}^{\prime}, \overline{\boldsymbol{x}}, \overline{\boldsymbol{z}}, \overline{\boldsymbol{z}}^{\prime}, \theta, \xi\right) \mathcal{W}_{2}\left(\boldsymbol{x}, \boldsymbol{z}, \boldsymbol{z}^{\prime}, \overline{\boldsymbol{x}}, \overline{\boldsymbol{z}}, \overline{\boldsymbol{z}}^{\prime}\right)\right. \\
\left.-\left(\boldsymbol{z}, \boldsymbol{z}^{\prime} \rightarrow \boldsymbol{y}\right)-\left(\overline{\boldsymbol{z}}, \overline{\boldsymbol{z}}^{\prime} \rightarrow \overline{\boldsymbol{y}}\right)+\left(\boldsymbol{z}, \boldsymbol{z}^{\prime} \rightarrow \boldsymbol{y} \& \overline{\boldsymbol{z}}, \overline{\boldsymbol{z}}^{\prime} \rightarrow \overline{\boldsymbol{y}}\right)\right]
\end{gathered}
$$

This equation involves the new tensorial kernel (the effective vertex $\Pi_{\lambda_{1} \lambda}^{i j m n}$ has been introduced in eq. (3.23))

$$
\mathcal{K}_{2}^{m n p q} \equiv \frac{\Pi_{\lambda_{1} \lambda}^{i j m n}\left(\boldsymbol{x}, \boldsymbol{z}, \boldsymbol{z}^{\prime}, \theta, \xi\right) \Pi_{\lambda_{1} \lambda}^{i j p q *}\left(\overline{\boldsymbol{x}}, \overline{\boldsymbol{z}}, \overline{\boldsymbol{z}}^{\prime}, \theta, \xi\right)}{\left[\theta^{2}(1-\theta) \boldsymbol{R}^{2}+\xi(\theta-\xi) \boldsymbol{Z}^{2}\right]\left[\theta^{2}(1-\theta) \overline{\boldsymbol{R}}^{2}+\xi(\theta-\xi) \overline{\boldsymbol{Z}}^{2}\right]},
$$

whose numerator can be explicitly computed as (as before, we exclude the contribution of the instantaneous pieces of the vertices, to simplify writing)

$$
\begin{aligned}
\left.\Pi_{\lambda_{1} \lambda}^{i j m n} \Pi_{\lambda_{1} \lambda}^{i j p q *}\right|_{\text {non-inst. }}= & 2 \xi(\theta-\xi) \theta^{2}(1-\theta)^{2} \\
& \times\left[(2-\theta)^{2} \delta^{l m} \delta^{r p}+\theta^{2}\left(\delta^{l r} \delta^{m p}-\delta^{l p} \delta^{m r}\right)\right]\left(\frac{2 \delta^{n l} \delta^{q r}}{\theta^{2}}+\frac{\delta^{n q} \delta^{l r}}{\xi^{2}}+\frac{\delta^{n q} \delta^{l r}}{(\theta-\xi)^{2}}\right) \\
= & 4 \xi(\theta-\xi) \theta^{2}(1-\theta)^{2}\left[\frac{(2-\theta)^{2} \delta^{n m} \delta^{q p}+\theta^{2} \delta^{m p} \delta^{n q}-\theta^{2} \delta^{n p} \delta^{q m}}{\theta^{2}}+\right. \\
& \left.+\delta^{n q} \delta^{m p}\left[1+(1-\theta)^{2}\right]\left(\frac{1}{\xi^{2}}+\frac{1}{(\theta-\xi)^{2}}\right)\right] .
\end{aligned}
$$

Furthermore, $\mathcal{W}_{2}$ is the following linear combination of partonic $S$-matrices:

$\mathcal{W}_{2}\left(\boldsymbol{x}, \boldsymbol{z}, \boldsymbol{z}^{\prime}, \overline{\boldsymbol{x}}, \overline{\boldsymbol{z}}, \overline{\boldsymbol{z}}^{\prime}\right)$

$$
\equiv S_{q g g \bar{q} g g}^{(2)}\left(\boldsymbol{x}, \boldsymbol{z}, \boldsymbol{z}^{\prime}, \overline{\boldsymbol{x}}, \overline{\boldsymbol{z}}, \overline{\boldsymbol{z}}^{\prime}\right)-S_{q g g \bar{q}}^{(2)}\left(\boldsymbol{x}, \boldsymbol{z}, \boldsymbol{z}^{\prime}, \overline{\boldsymbol{w}}\right)-S_{q \bar{q} g g}^{(2)}\left(\boldsymbol{w}, \overline{\boldsymbol{x}}, \overline{\boldsymbol{z}}, \overline{\boldsymbol{z}}^{\prime}\right)+\mathcal{S}(\boldsymbol{w}, \overline{\boldsymbol{w}}),
$$


where the 6-parton $S$-matrix describes final-state interactions in both the DA and the CCA,

$$
\begin{aligned}
& S_{q g g \bar{q} g g}^{(2)}\left(\boldsymbol{x}, \boldsymbol{z}, \boldsymbol{z}^{\prime}, \overline{\boldsymbol{x}}, \overline{\boldsymbol{z}}, \overline{\boldsymbol{z}}^{\prime}\right) \\
& \equiv \frac{1}{C_{F} N_{c}^{2}} f^{r m n} f^{a b c}\left\langle\left[U^{\dagger}(\overline{\boldsymbol{z}}) U(\boldsymbol{z})\right]^{n c}\left[U^{\dagger}\left(\overline{\boldsymbol{z}}^{\prime}\right) U\left(\boldsymbol{z}^{\prime}\right)\right]^{m b} \operatorname{tr}\left[V^{\dagger}(\overline{\boldsymbol{x}}) V(\boldsymbol{x}) t^{a} t^{r}\right]\right\rangle \\
& \simeq \frac{1}{2}\left[\mathcal{Q}\left(\boldsymbol{z}, \boldsymbol{z}^{\prime}, \overline{\boldsymbol{z}}^{\prime}, \overline{\boldsymbol{z}}\right) \mathcal{Q}(\boldsymbol{x}, \boldsymbol{z}, \overline{\boldsymbol{z}}, \overline{\boldsymbol{x}}) \mathcal{S}\left(\boldsymbol{z}^{\prime}, \overline{\boldsymbol{z}}^{\prime}\right)+\mathcal{Q}\left(\boldsymbol{z}^{\prime}, \boldsymbol{z}, \overline{\boldsymbol{z}}, \overline{\boldsymbol{z}}^{\prime}\right) \mathcal{Q}\left(\boldsymbol{x}, \boldsymbol{z}^{\prime}, \overline{\boldsymbol{z}}^{\prime}, \overline{\boldsymbol{x}}\right) \mathcal{S}(\boldsymbol{z}, \overline{\boldsymbol{z}})\right],
\end{aligned}
$$

(the two terms correspond to permutations of the final gluons), whereas the 4-parton $S$ matrix describes the interference between scattering in the final state and the initial state, respectively:

$$
\begin{aligned}
S_{q g g \bar{q}}^{(2)}\left(\boldsymbol{x}, \boldsymbol{z}, \boldsymbol{z}^{\prime}, \overline{\boldsymbol{w}}\right) & \equiv \frac{1}{C_{F} N_{c}^{2}} f^{a b c} f^{r d e}\left\langle U^{e c}\left(\boldsymbol{z}^{\prime}\right) U^{d b}(\boldsymbol{z}) \operatorname{tr}\left[V^{\dagger}(\overline{\boldsymbol{w}}) t^{r} V(\boldsymbol{x}) t^{a}\right]\right\rangle \\
& \simeq \frac{1}{2}\left[\mathcal{S}\left(\boldsymbol{x}, \boldsymbol{z}^{\prime}\right) \mathcal{S}\left(\boldsymbol{z}^{\prime}, \boldsymbol{z}\right) \mathcal{S}(\boldsymbol{z}, \overline{\boldsymbol{w}})+\mathcal{S}(\boldsymbol{x}, \boldsymbol{z}) \mathcal{S}\left(\boldsymbol{z}, \boldsymbol{z}^{\prime}\right) \mathcal{S}\left(\boldsymbol{z}^{\prime}, \overline{\boldsymbol{w}}\right)\right]
\end{aligned}
$$

As a check, one can see that after performing the double replacement $\left(\boldsymbol{z}, \boldsymbol{z}^{\prime} \rightarrow \boldsymbol{y} \& \overline{\boldsymbol{z}}, \overline{\boldsymbol{z}}^{\prime} \rightarrow\right.$ $\overline{\boldsymbol{y}})$ in eq. (3.49), one recovers the colour structure describing the scattering of a quark-gluon pair (the structure shown in eq. (3.36) at large $N_{c}$ ).

Note that, as compared to eq. (3.39), eq. (3.46) contains an additional factor of 4 (besides the modified colour factor, which reflects the different structure of the partonic $S$-matrices). This factor of 4 is related to the symmetry of the amplitude in figure 7 under the exchange of the two final gluons. This in turn implies that the integrand of eq. (3.46) is symmetric under the simultaneous exchanges $\boldsymbol{z} \leftrightarrow \boldsymbol{z}^{\prime}, \overline{\boldsymbol{z}} \leftrightarrow \overline{\boldsymbol{z}}^{\prime}$, and $\xi \leftrightarrow \theta-\xi$, as it can be easily verified. We used this symmetry property twice: (i) for a given assignment of the momenta of the two gluons (e.g., $k_{2}^{+}=\xi q^{+}$and $k_{3}^{+}=(\theta-\xi) q^{+}$, as in figure 10), there are two possible Wick contractions in the calculation of the expectation value $\hat{\mathcal{N}}_{g}\left(k_{2}\right) \hat{\mathcal{N}}_{g}\left(k_{3}\right)$, cf. eq. (3.38), which give identical results; (ii) the graphs obtained by permuting the gluon momenta, $k_{2} \leftrightarrow k_{3}$, give identical results as well, because we can undo the effect of this permutation via a change of variables which leaves the integrand unchanged.

The remaining terms refer to interferences between the two topologies shown in figure 6 and figure 7, respectively. We shall explicitly show the piece where the topology in figure 6 counts for the DA and that in figure 7, for the CCA (see figure 11 for an illustration). The total result can be then obtained by taking the double of the real part of the shown 


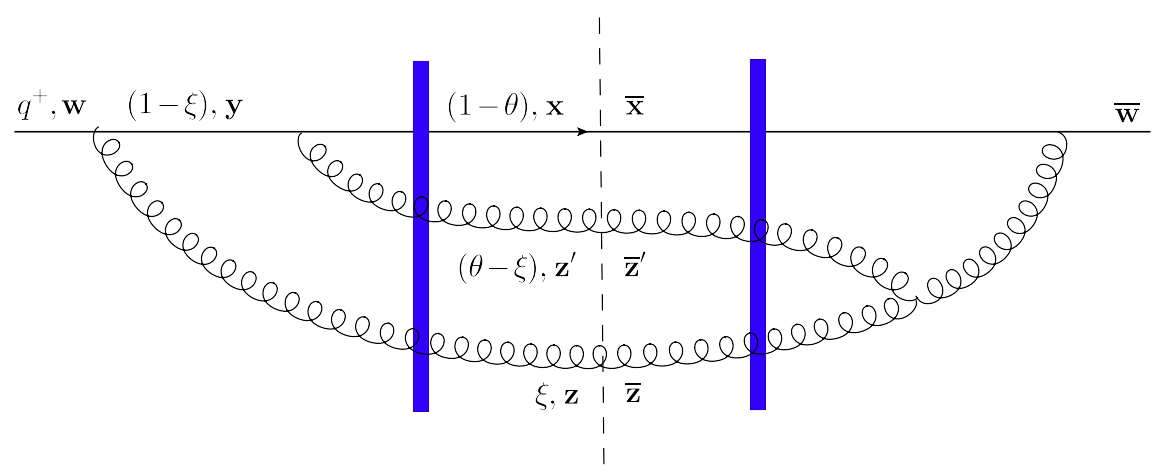

Figure 11. A particular graph contributing to the "interference" cross-section (3.52); its colour structure is exhibited in eq. (3.55).

contribution. One finds

$$
\begin{aligned}
\frac{d \sigma_{(3)}^{q A \rightarrow q g g+X}}{d k_{1}^{+} d^{2} \boldsymbol{k}_{1} d k_{2}^{+} d^{2} \boldsymbol{k}_{2} d k_{3}^{+} d^{2} \boldsymbol{k}_{3}}=\frac{2 \alpha_{s}^{2} C_{F} N_{c}}{4(2 \pi)^{10}\left(q^{+}\right)^{2}} \delta\left(q^{+}-k_{1}^{+}-k_{2}^{+}-k_{3}^{+}\right) \\
\times 2 \mathcal{R} e \int_{\overline{\boldsymbol{x}}, \overline{\boldsymbol{z}}, \overline{\boldsymbol{z}}^{\prime}, \boldsymbol{x}, \boldsymbol{z}, \boldsymbol{z}^{\prime}} e^{-i \boldsymbol{k}_{1} \cdot(\boldsymbol{x}-\overline{\boldsymbol{x}})-i \boldsymbol{k}_{2} \cdot(\boldsymbol{z}-\overline{\boldsymbol{z}})-i \boldsymbol{k}_{3} \cdot\left(\boldsymbol{z}^{\prime}-\overline{\boldsymbol{z}}^{\prime}\right)} \frac{\boldsymbol{Y}^{m}\left(\boldsymbol{X}^{\prime}\right)^{n} \overline{\boldsymbol{R}}^{p} \overline{\boldsymbol{Z}}^{q}}{\left(\boldsymbol{X}^{\prime}\right)^{2} \overline{\boldsymbol{Z}}^{2}} \\
\times\left[\mathcal{K}_{3}^{m n p q}\left(\boldsymbol{x}, \boldsymbol{z}, \boldsymbol{z}^{\prime}, \overline{\boldsymbol{x}}, \overline{\boldsymbol{z}}, \overline{\boldsymbol{z}}^{\prime}, \theta, \xi\right) \mathcal{W}_{3}\left(\boldsymbol{x}, \boldsymbol{z}, \boldsymbol{z}^{\prime}, \overline{\boldsymbol{x}}, \overline{\boldsymbol{z}}, \overline{\boldsymbol{z}}^{\prime}\right)\right. \\
\left.-\left(\boldsymbol{x}, \boldsymbol{z}^{\prime} \rightarrow \boldsymbol{y}\right)-\left(\overline{\boldsymbol{z}}, \overline{\boldsymbol{z}}^{\prime} \rightarrow \overline{\boldsymbol{y}}\right)+\left(\boldsymbol{x}, \boldsymbol{z}^{\prime} \rightarrow \boldsymbol{y} \& \overline{\boldsymbol{z}}, \overline{\boldsymbol{z}}^{\prime} \rightarrow \overline{\boldsymbol{y}}\right)\right] \\
+\left(k_{2}^{+} \leftrightarrow k_{3}^{+}, \boldsymbol{k}_{2} \leftrightarrow \boldsymbol{k}_{3}\right) .
\end{aligned}
$$

It is important to notice that, in the subtracted terms, $\boldsymbol{y}$ is the function of $\boldsymbol{x}$ and $\boldsymbol{z}^{\prime}$ defined in eq. (3.16), whereas $\overline{\boldsymbol{y}}$ is rather a function of $\overline{\boldsymbol{z}}$ and $\overline{\boldsymbol{z}}^{\prime}$ defined by the "bared" version of eq. (3.5). Furthermore,

$$
\mathcal{K}_{3}^{m n p q} \equiv \frac{\Xi_{\lambda_{1} \lambda}^{i j m n}\left(\boldsymbol{x}, \boldsymbol{z}, \boldsymbol{z}^{\prime}, \theta, \xi\right) \Pi_{\lambda_{1} \lambda}^{i j p q *}\left(\overline{\boldsymbol{x}}, \overline{\boldsymbol{z}}, \overline{\boldsymbol{z}}^{\prime}, \theta, \xi\right)}{\left[(1-\theta)(\theta-\xi)\left(\boldsymbol{X}^{\prime}\right)^{2}+\xi(1-\xi)^{2} \boldsymbol{Y}^{2}\right]\left[\theta^{2}(1-\theta) \overline{\boldsymbol{R}}^{2}+\xi(\theta-\xi) \overline{\boldsymbol{Z}}^{2}\right]} .
$$

The combination of Wilson lines describing the scattering has the same general structure, that is,

$$
\begin{aligned}
& \mathcal{W}_{3}\left(\boldsymbol{x}, \boldsymbol{z}, \boldsymbol{z}^{\prime}, \overline{\boldsymbol{x}}, \overline{\boldsymbol{z}}, \overline{\boldsymbol{z}}^{\prime}\right) \\
& \equiv S_{q g g \bar{q} g g}^{(3)}\left(\boldsymbol{x}, \boldsymbol{z}, \boldsymbol{z}^{\prime}, \overline{\boldsymbol{x}}, \overline{\boldsymbol{z}}, \overline{\boldsymbol{z}}^{\prime}\right)-S_{q g g \bar{q}}^{(3)}\left(\boldsymbol{x}, \boldsymbol{z}, \boldsymbol{z}^{\prime}, \overline{\boldsymbol{w}}\right)-S_{q \bar{q} g g}^{(3)}\left(\boldsymbol{w}, \overline{\boldsymbol{x}}, \overline{\boldsymbol{z}}, \overline{\boldsymbol{z}}^{\prime}\right)+\mathcal{S}(\boldsymbol{w}, \overline{\boldsymbol{w}}),
\end{aligned}
$$

with however some new ingredients, namely,

$$
\begin{aligned}
& S_{q g g \bar{q} g g}^{(3)}\left(\boldsymbol{x}, \boldsymbol{z}, \boldsymbol{z}^{\prime}, \overline{\boldsymbol{x}}, \overline{\boldsymbol{z}}, \overline{\boldsymbol{z}}^{\prime}\right) \\
& \quad \equiv \frac{2 i}{C_{F} N_{c}^{2}} f^{c d e}\left\langle\left[U^{\dagger}(\overline{\boldsymbol{z}}) U(\boldsymbol{z})\right]^{d a}\left[U^{\dagger}\left(\overline{\boldsymbol{z}}^{\prime}\right) U\left(\boldsymbol{z}^{\prime}\right)\right]^{e b} \operatorname{tr}\left[V^{\dagger}(\overline{\boldsymbol{x}}) V(\boldsymbol{x}) t^{b} t^{a} t^{c}\right]\right\rangle \\
& \quad \simeq \mathcal{Q}\left(\boldsymbol{x}, \boldsymbol{z}^{\prime}, \overline{\boldsymbol{z}}^{\prime}, \overline{\boldsymbol{x}}\right) \mathcal{Q}\left(\boldsymbol{z}^{\prime}, \boldsymbol{z}, \overline{\boldsymbol{z}}, \overline{\boldsymbol{z}}^{\prime}\right) \mathcal{S}(\boldsymbol{z}, \overline{\boldsymbol{z}}),
\end{aligned}
$$




$$
\begin{aligned}
S_{q g g \bar{q}}^{(3)}\left(\boldsymbol{x}, \boldsymbol{z}, \boldsymbol{z}^{\prime}, \overline{\boldsymbol{w}},\right) \equiv & \frac{2 i}{C_{F} N_{c}^{2}} f^{e c d}\left\langle U^{c a}(\boldsymbol{z}) U^{d b}\left(\boldsymbol{z}^{\prime}\right) \operatorname{tr}\left[V^{\dagger}(\overline{\boldsymbol{w}}) t^{e} V(\boldsymbol{x}) t^{b} t^{a}\right]\right\rangle \\
& \simeq \mathcal{S}\left(\boldsymbol{x}, \boldsymbol{z}^{\prime}\right) \mathcal{S}\left(\boldsymbol{z}^{\prime}, \boldsymbol{z}\right) \mathcal{S}(\boldsymbol{z}, \overline{\boldsymbol{w}}), \\
S_{q \bar{q} g g}^{(3)}\left(\boldsymbol{w}, \overline{\boldsymbol{x}}, \overline{\boldsymbol{z}}, \overline{\boldsymbol{z}}^{\prime}\right) \equiv & \frac{2 i}{C_{F} N_{c}^{2}} f^{e c d}\left\langle U^{c a}(\overline{\boldsymbol{z}}) U^{b d}\left(\overline{\boldsymbol{z}}^{\prime}\right) \operatorname{tr}\left[V^{\dagger}(\overline{\boldsymbol{x}}) t^{b} t^{a} V(\boldsymbol{w}) t^{e}\right]\right\rangle \\
& \simeq \mathcal{S}(\boldsymbol{w}, \overline{\boldsymbol{z}}) \mathcal{S}\left(\overline{\boldsymbol{z}}, \overline{\boldsymbol{z}}^{\prime}\right) \mathcal{S}\left(\overline{\boldsymbol{z}}^{\prime}, \overline{\boldsymbol{x}}\right) .
\end{aligned}
$$

It might be interesting to notice that the last term in eq. (3.52), as obtained via the double replacement $\left(\boldsymbol{x}, \boldsymbol{z}^{\prime} \rightarrow \boldsymbol{y} \& \overline{\boldsymbol{z}}, \overline{\boldsymbol{z}}^{\prime} \rightarrow \overline{\boldsymbol{y}}\right)$, does not has the same colour structure as expected for a final quark-gluon state (unlike the respective limits for all the other colour functions $\mathcal{W}_{0}, \mathcal{W}_{1}$, and $\mathcal{W}_{2}$ ). One finds indeed

$$
\mathcal{W}_{3}(\boldsymbol{y}, \boldsymbol{z}, \boldsymbol{y}, \overline{\boldsymbol{x}}, \overline{\boldsymbol{y}}, \overline{\boldsymbol{y}}) \simeq \mathcal{S}(\boldsymbol{y}, \boldsymbol{z}) \mathcal{S}(\overline{\boldsymbol{y}}, \overline{\boldsymbol{x}}) \mathcal{S}(\boldsymbol{z}, \overline{\boldsymbol{y}})-\mathcal{S}(\boldsymbol{y}, \boldsymbol{z}) \mathcal{S}(\boldsymbol{z}, \overline{\boldsymbol{w}})-\mathcal{S}(\boldsymbol{w}, \overline{\boldsymbol{y}}) \mathcal{S}(\overline{\boldsymbol{y}}, \overline{\boldsymbol{x}})+\mathcal{S}(\boldsymbol{w}, \overline{\boldsymbol{w}}),
$$

which should be compared to eq. (3.36). This difference is due to the fact that the pattern of the colour flow in the interference graphs is different as compared to the direct graphs.

Note finally that, in the large- $N_{c}$ limit, the three functions encoding the colour structure in the various contributions to the cross-section, namely $\mathcal{W}_{1}, \mathcal{W}_{2}$, and $\mathcal{W}_{3}$, become very similar to each other: $\mathcal{W}_{1}$ and $\mathcal{W}_{3}$ take exactly the same form, shown in eq. (3.45), whereas $\mathcal{W}_{2}$ differs only through the additional symmetrisation with respect to the exchange of the two final gluons.

\section{Next-to-leading order corrections: the real terms}

As mentioned in the Introduction, the next-to-leading order (NLO) corrections to the crosssection for forward dijet production in $p A$ collisions can be divided into two classes: real and virtual. In the remaining part of this paper, we shall compute the real corrections - those associated with a final state which involves three partons ("jets"), out of which only two are measured. By integrating out the kinematics of the unmeasured parton, one generates a loop correction to the cross-section for the two measured jets. This loop opens in the direct amplitude (DA) and closes back in the complex conjugate amplitude (CCA). The virtual NLO corrections, on the other hand, refer to loop corrections to the amplitude itself. They will be addressed in a subsequent paper.

\subsection{The di-quark jet production}

We first consider final states which include two measured fermions: two quarks, or a quarkantiquark pair. The cross-section for di-quark jet production ${ }^{7}$ is obtained by "integrating

\footnotetext{
${ }^{7}$ This particular channel, i.e. $q A \rightarrow q q+X$, does not exist at leading-order, unless one considers double quark scattering, that is, the simultaneous scattering and production of two collinear quarks from the wavefunction of the incoming proton. The respective contribution counts at zeroth order in $\alpha_{s}$, but it is proportional to the double-quark distribution in the proton (that can be roughly estimated as the product of two standard, single-quark, distributions).
} 
out" the final antiquark in our general formula for the three-quark final state, cf. eq. (3.27):

$$
\frac{d \sigma_{\mathrm{rNLO}}^{q A \rightarrow q q+X}}{d k_{1}^{+} d^{2} \boldsymbol{k}_{1} d k_{2}^{+} d^{2} \boldsymbol{k}_{2}}=\int d k_{3}^{+} d^{2} \boldsymbol{k}_{3} \frac{d \sigma^{q A \rightarrow q q \bar{q}+X}}{d k_{1}^{+} d^{2} \boldsymbol{k}_{1} d k_{2}^{+} d^{2} \boldsymbol{k}_{2} d k_{3}^{+} d^{2} \boldsymbol{k}_{3}} .
$$

(The subscript "rNLO" stays for real next-to-leading order corrections.) With reference to eq. (3.27), it is quite clear that the integral over $k_{3}^{+}$can be trivially performed by using the $\delta$-function for longitudinal momentum conservation, whereas the integral over $\boldsymbol{k}_{3}$ yields a factor $(2 \pi)^{2} \delta^{(2)}\left(\boldsymbol{z}^{\prime}-\overline{\boldsymbol{z}}^{\prime}\right)$, which allows one to identify the coordinates $\boldsymbol{z}^{\prime}$ and $\overline{\boldsymbol{z}}^{\prime}$ of the unmeasured antiquark in the DA and the CCA, respectively. The result of eq. (4.1) can be succinctly written as

$$
\frac{d \sigma_{\mathrm{rNLO}}^{q A \rightarrow q q+X}}{d k_{1}^{+} d^{2} \boldsymbol{k}_{1} d k_{2}^{+} d^{2} \boldsymbol{k}_{2}}=\left.(2 \pi)^{2} \frac{d \sigma^{q A \rightarrow q q \bar{q}+X}}{d k_{1}^{+} d^{2} \boldsymbol{k}_{1} d k_{2}^{+} d^{2} \boldsymbol{k}_{2} d k_{3}^{+} d^{2} \boldsymbol{k}_{3}}\right|_{k_{3}^{+}=q^{+-} k_{1}^{+}-k_{2}^{+}, \boldsymbol{z}^{\prime}=\overline{\boldsymbol{z}}^{\prime}},
$$

where it is understood that the trijet cross-section in the r.h.s. is given by eq. (3.27), but without the $\delta$-function expressing the conservation of longitudinal momentum.

Some care must be taken, concerning the order of limits: the identification $\boldsymbol{z}^{\prime}=\overline{\boldsymbol{z}}^{\prime}$ must be made only after performing the subtractions which occur in the integrand of eq. (3.27). For instance, in the subtracted term denoted as $\left(\overline{\boldsymbol{z}}, \overline{\boldsymbol{z}}^{\prime} \rightarrow \overline{\boldsymbol{y}}\right)$, one must first perform the replacements $\overline{\boldsymbol{z}} \rightarrow \overline{\boldsymbol{y}}$ and $\overline{\boldsymbol{z}}^{\prime} \rightarrow \overline{\boldsymbol{y}}$ at fixed $\boldsymbol{z}^{\prime}$ and only then identify $\overline{\boldsymbol{z}}^{\prime}$ with $\boldsymbol{z}^{\prime}$. (The two limits do not commute with each other, as one can easily check.)

The fact that the antiquark is not measured brings some simplifications in the structure of the 6-quark $S$-matrix in eq. (3.33) (which describes final-state interactions, cf. figure 8left): when $\boldsymbol{z}^{\prime}=\overline{\boldsymbol{z}}^{\prime}$, the Wilson lines describing the scattering of the antiquark compensate each other by unitarity, $V^{\dagger}\left(\boldsymbol{z}^{\prime}\right) V\left(\boldsymbol{z}^{\prime}\right)=1$, and then the quadrupole appearing in the second line of eq. (3.33) reduces to a dipole (we consider large $N_{c}$, for simplicity):

$$
S_{q q \bar{q} \bar{q} \bar{q}}\left(\boldsymbol{x}, \boldsymbol{z}, \boldsymbol{z}^{\prime}, \overline{\boldsymbol{x}}, \overline{\boldsymbol{z}}, \overline{\boldsymbol{z}}^{\prime}=\boldsymbol{z}^{\prime}\right) \simeq \mathcal{S}(\boldsymbol{x}, \overline{\boldsymbol{x}}) \mathcal{S}(\boldsymbol{z}, \overline{\boldsymbol{z}}),
$$

However, this simplification refers only to the first term within the square brackets of eq. (3.27). Consider e.g. the second term, denoted as $\left(\boldsymbol{z}, \boldsymbol{z}^{\prime} \rightarrow \boldsymbol{y}\right)$. If one first replaces both $\boldsymbol{z}$ and $\boldsymbol{z}^{\prime}$ by $\boldsymbol{y}$, and only then one identifies $\boldsymbol{z}^{\prime}=\overline{\boldsymbol{z}}^{\prime}$, then the quadrupole structure survives in eq. (3.33):

$$
S_{q q \bar{q} \bar{q} q}\left(\boldsymbol{x}, \boldsymbol{y}, \boldsymbol{y}, \overline{\boldsymbol{x}}, \overline{\boldsymbol{z}}, \overline{\boldsymbol{z}}^{\prime}=\boldsymbol{z}^{\prime}\right) \simeq \mathcal{O}\left(\boldsymbol{x}, \boldsymbol{y}, \boldsymbol{z}^{\prime}, \overline{\boldsymbol{x}}\right) \mathcal{S}(\boldsymbol{y}, \overline{\boldsymbol{z}})
$$

In this equation, $\boldsymbol{y}$ is understood as the function of $\boldsymbol{z}$ and $\boldsymbol{z}^{\prime}$ shown in eq. (3.5).

The longitudinal momentum fractions $\theta$ and $\xi$ which implicitly appear in the r.h.s. of eq. (4.2) are fixed by eq. (3.29), which for the present purposes should be rewritten in terms of the longitudinal momentum fractions $x_{1} \equiv k_{1}^{+} / Q^{+}$and $x_{2} \equiv k_{2}^{+} / Q^{+}$of the measured partons ("jets") and the respective fraction $x_{q} \equiv q^{+} / Q^{+}$of the original quark:

$$
\theta=1-\frac{k_{1}^{+}}{q^{+}}=1-\frac{x_{1}}{x_{q}}, \quad \xi=\frac{k_{2}^{+}}{q^{+}}=\frac{x_{2}}{x_{q}} .
$$


As discussed in relation with eq. (2.16), the physical dijet cross-section (to the order of interest) is obtained by averaging over $x_{q}$ with the quark distribution inside the proton:

$$
\left.\frac{d \sigma_{\mathrm{rNLO}}^{p A \rightarrow 2 j e t+X}}{d^{3} k d^{3} p}\right|_{q \rightarrow q q}=\int d x_{q} q_{f}\left(x_{q}, \mu^{2}\right) \Theta\left(x_{q}-x_{1}-x_{2}\right) \frac{d \sigma_{\mathrm{rNLO}}^{q A \rightarrow q q+X}}{d k_{1}^{+} d^{2} \boldsymbol{k}_{1} d k_{2}^{+} d^{2} \boldsymbol{k}_{2}} .
$$

It is furthermore important to keep in mind that the cross-section (4.6) depends upon the "plus" longitudinal fractions $x_{1}, x_{2}$, and $x_{q}$ not only via its dependence upon $\theta$ and $\xi$, as explicit in eq. (3.30) for the kernel $\mathcal{K}_{0}$, but also via the dependence of the various $S$-matrices upon the "rapidity" variable ${ }^{8} x_{g}$, as introduced by their high-energy evolution. We recall that $x_{g}$ is the "minus" longitudinal momentum fraction carried by the gluons from the nucleus which participate in the scattering. This is indeed a function of $x_{1}, x_{2}$ and $x_{3}=x_{q}-x_{1}-x_{2}$, as shown in eq. (3.37).

\subsection{The quark-antiquark di-jets}

The case where the measured dijet is made with a quark-antiquark pair ${ }^{9}$ is similarly obtained by integrating eq. (3.27) over the kinematics of one of the two final quarks, e.g. over $k_{1}$. This gives

$$
\frac{d \sigma_{\mathrm{rNLO}}^{q A \rightarrow q \bar{q}+X}}{d k_{2}^{+} d^{2} \boldsymbol{k}_{2} d k_{3}^{+} d^{2} \boldsymbol{k}_{3}}=\int d k_{1}^{+} d^{2} \boldsymbol{k}_{1} \frac{d \sigma^{q A \rightarrow q q \bar{q}+X}}{d k_{1}^{+} d^{2} \boldsymbol{k}_{1} d k_{2}^{+} d^{2} \boldsymbol{k}_{2} d k_{3}^{+} d^{2} \boldsymbol{k}_{3}} .
$$

The above r.h.s. involves two contributions: the unmeasured quark $k_{1}$ can be either the incoming quark, or the quark produced by the decay of the intermediate gluon. The contribution of the first case to the $q q \bar{q}$ cross-section is explicitly shown in eq. (3.27), while that of the second case can be obtained by exchanging $k_{1}$ and $k_{2}$ within the first contribution.

Specifically, in the first case ( $k_{1}$ corresponds to the leading quark), the integral over $\boldsymbol{k}_{1}$ enforces $\boldsymbol{x}=\overline{\boldsymbol{x}}$ and the longitudinal momentum fractions should be evaluated as

$$
\theta=1-\frac{x_{2}+x_{3}}{x_{q}}, \quad \xi=\frac{x_{2}}{x_{q}} .
$$

The fact that $\boldsymbol{x}=\overline{\boldsymbol{x}}$ simplifies the colour structure of the cross-sections in all the situations where the leading quark interacts in both the DA and the CCA: the respective Wilson lines compensate each other by unitarity. In what follows we shall exhibit these simplifications directly in the large $N_{c}$ limit.

Specifically, the 6-quark $S$-matrix $S_{q q \bar{q} \bar{q} q}$ (3.33) reduces to a product of two dipoles (one for each of the two measured partons):

$$
S_{q q \bar{q} \bar{q} q}\left(\boldsymbol{x}, \boldsymbol{z}, \boldsymbol{z}^{\prime}, \overline{\boldsymbol{x}}=\boldsymbol{x}, \overline{\boldsymbol{z}}, \overline{\boldsymbol{z}}^{\prime}\right) \simeq \mathcal{S}\left(\overline{\boldsymbol{z}}^{\prime}, \boldsymbol{z}^{\prime}\right) \mathcal{S}(\boldsymbol{z}, \overline{\boldsymbol{z}}) .
$$

\footnotetext{
${ }^{8}$ More precisely, the evolution "rapidity" related to $x_{g}$ is $Y_{g} \equiv \ln \left(1 / x_{g}\right)$.

${ }^{9}$ This case can be viewed as a NLO correction to the $\mathcal{O}\left(\alpha_{s}\right)$-process in which a gluon collinear with the incoming parton splits into a $q \bar{q}$ pair while scattering off the nuclear target $[1,53]$. The respective cross-section involves the gluon distribution, which is however suppressed at large $x$, i.e. for the forward kinematics of interest for us here.
} 
A similar simplification occurs in all the four terms within the squared brackets in eq. (3.27); e.g., for the last term, as obtained via the double replacement $\left(\boldsymbol{z}, \boldsymbol{z}^{\prime} \rightarrow \boldsymbol{y} \& \overline{\boldsymbol{z}}, \overline{\boldsymbol{z}}^{\prime} \rightarrow \overline{\boldsymbol{y}}\right)$, one finds

$$
S_{q q \bar{q} \bar{q} q}(\boldsymbol{x}, \boldsymbol{y}, \boldsymbol{y}, \overline{\boldsymbol{x}}=\boldsymbol{x}, \overline{\boldsymbol{y}}, \overline{\boldsymbol{y}}) \simeq \mathcal{S}(\overline{\boldsymbol{y}}, \boldsymbol{y}) \mathcal{S}(\boldsymbol{y}, \overline{\boldsymbol{y}}) .
$$

This is recognised as the large- $N_{c}$ version of the $S$-matrix for a colour dipole made with two gluons.

In the second case, where $k_{1}$ refers to the quark originating from the gluon decay, the integral over $\boldsymbol{k}_{1}$ enforces $\boldsymbol{z}=\overline{\boldsymbol{z}}$ and the longitudinal momentum fractions are evaluated as

$$
\theta=1-\frac{x_{2}}{x_{q}}, \quad \xi=1-\frac{x_{2}+x_{3}}{x_{q}} .
$$

As in the case of eq. (4.2), the limit $\boldsymbol{z} \rightarrow \overline{\boldsymbol{z}}$ does not commute with the various replacements in eq. (3.27), like $\left(\boldsymbol{z}, \boldsymbol{z}^{\prime} \rightarrow \boldsymbol{y}\right)$, and must be performed after them. Accordingly, there is only one simplification in the colour structure, corresponding to the unique topology (in terms of shockwave insertions) in which the unmeasured gluon $k_{1}$ scatters both in the DA and in the CCA. One then has

$$
S_{q q \bar{q} \bar{q} q q}\left(\boldsymbol{x}, \boldsymbol{z}, \boldsymbol{z}^{\prime}, \overline{\boldsymbol{x}}, \overline{\boldsymbol{z}}=\boldsymbol{z}, \overline{\boldsymbol{z}}^{\prime}\right) \simeq \mathcal{Q}\left(\boldsymbol{x}, \boldsymbol{z}^{\prime}, \overline{\boldsymbol{z}}^{\prime}, \overline{\boldsymbol{x}}\right) .
$$

\subsection{The two-gluon di-jets}

The cross-section for the final state involving two measured gluons is obtained by "integrating out" the final quark in the trijet cross-section corresponding to the $q g g$ final state. As discussed in section 3.2.2, there are three contributions to the $q g g$ cross-section, illustrated in figures 9$),(10$, and 11. By integrating out these three contributions over the kinematics $\left(k_{1}^{+}, \boldsymbol{k}_{1}\right)$ of the unmeasured quark, one finds

$$
\frac{d \sigma_{\mathrm{rNLO}}^{q A \rightarrow g g+X}}{d k_{2}^{+} d^{2} \boldsymbol{k}_{2} d k_{3}^{+} d^{2} \boldsymbol{k}_{3}}=\left.(2 \pi)^{2} \sum_{i=1,2,3} \frac{d \sigma_{(i)}^{q A \rightarrow q g g+X}}{d k_{1}^{+} d^{2} \boldsymbol{k}_{1} d k_{2}^{+} d^{2} \boldsymbol{k}_{2} d k_{3}^{+} d^{2} \boldsymbol{k}_{3}}\right|_{k_{1}^{+}=q^{+}-k_{2}^{+}-k_{3}^{+}, \boldsymbol{x}=\overline{\boldsymbol{x}}} .
$$

We again used the compact but somewhat formal notation introduced in eq. (4.2), where it is understood that the $\delta$-function expressing longitudinal momentum conservation is excluded from the trijet cross-sections. In turn, each of the three terms in the r.h.s. is made with two pieces, corresponding to the permutations of the measured gluons $k_{2}$ and $k_{3}$.

It is further instructive to notice the simplifications in the colour structure of the trijet cross-sections which appear due to the fact that the quark is not measured $(\boldsymbol{x}=\overline{\boldsymbol{x}})$. These simplifications refer to the $S$-matrices denoted as $S_{q g g \bar{q} g g}^{(i)}$ with $i=1,2,3$, which describe situations where the final quark scatters both in the DA and in the CCA. As usual, we show the ensuing simplifications only at large $N_{c}$. In the three cases, one of the two quadrupole factors reduces to a dipole. One finds, ${ }^{10}$

$$
\begin{aligned}
S_{q g g \bar{q} g g}^{(1)}\left(\boldsymbol{x}, \boldsymbol{z}, \boldsymbol{z}^{\prime}, \overline{\boldsymbol{x}}=\boldsymbol{x}, \overline{\boldsymbol{z}}, \overline{\boldsymbol{z}}^{\prime}\right) & \simeq S_{q g g \bar{q} g g}^{(3)}\left(\boldsymbol{x}, \boldsymbol{z}, \boldsymbol{z}^{\prime}, \overline{\boldsymbol{x}}=\boldsymbol{x}, \overline{\boldsymbol{z}}, \overline{\boldsymbol{z}}^{\prime}\right) \\
& \simeq \mathcal{S}\left(\overline{\boldsymbol{z}}^{\prime}, \boldsymbol{z}^{\prime}\right) \mathcal{Q}\left(\boldsymbol{z}^{\prime}, \boldsymbol{z}, \overline{\boldsymbol{z}}, \overline{\boldsymbol{z}}^{\prime}\right) \mathcal{S}(\boldsymbol{z}, \overline{\boldsymbol{z}}) .
\end{aligned}
$$

\footnotetext{
${ }^{10}$ As implied by its notation, the colour dipole $\mathcal{S}\left(\overline{\boldsymbol{z}}^{\prime}, \boldsymbol{z}^{\prime}\right)$ is built with a quark located at $\overline{\boldsymbol{z}}^{\prime}$ in the CCA and an anti-quark located at $\boldsymbol{z}^{\prime}$ in the DA; recall eq. (2.12).
} 
and respectively

$$
\begin{aligned}
S_{q g g \bar{q} g g}^{(2)}\left(\boldsymbol{x}, \boldsymbol{z}, \boldsymbol{z}^{\prime}, \overline{\boldsymbol{x}}=\boldsymbol{x}, \overline{\boldsymbol{z}}, \overline{\boldsymbol{z}}^{\prime}\right) \simeq & \frac{1}{2}\left[\mathcal{S}(\overline{\boldsymbol{z}}, \boldsymbol{z}) \mathcal{Q}\left(\boldsymbol{z}, \boldsymbol{z}^{\prime}, \overline{\boldsymbol{z}}^{\prime}, \overline{\boldsymbol{z}}\right) \mathcal{S}\left(\boldsymbol{z}^{\prime}, \overline{\boldsymbol{z}}^{\prime}\right)\right. \\
& \left.+\mathcal{S}\left(\overline{\boldsymbol{z}}^{\prime}, \boldsymbol{z}^{\prime}\right) \mathcal{Q}\left(\boldsymbol{z}^{\prime}, \boldsymbol{z}, \overline{\boldsymbol{z}}, \overline{\boldsymbol{z}}^{\prime}\right) \mathcal{S}(\boldsymbol{z}, \overline{\boldsymbol{z}})\right]
\end{aligned}
$$

\subsection{The quark-gluon dijets}

The quark-gluon dijet final state already exists at leading order, as discussed in section 2 . The corresponding real NLO corrections are obtained by integrating out one of the two final gluons from the $q g g$ trijet cross-section computed in section 3.2.2, say the one with momentum $k_{3}$. The complete result is therefore the sum of three terms,

$$
\frac{d \sigma_{\mathrm{rNLO}}^{q A \rightarrow q g+X}}{d k_{1}^{+} d^{2} \boldsymbol{k}_{1} d k_{2}^{+} d^{2} \boldsymbol{k}_{2}}=\int d k_{3}^{+} d^{2} \boldsymbol{k}_{3} \sum_{i=1,2,3} \frac{d \sigma_{(i)}^{q A \rightarrow q g g+X}}{d k_{1}^{+} d^{2} \boldsymbol{k}_{1} d k_{2}^{+} d^{2} \boldsymbol{k}_{2} d k_{3}^{+} d^{2} \boldsymbol{k}_{3}}
$$

In turn, each of these three terms is made with two pieces, since the unmeasured gluon with momentum $k_{3}$ can be any of the two final gluons in figures 9$),(10$, and 11. Consider for definiteness the case where this is the gluon with longitudinal momentum $\theta-\xi$ and transverse coordinates $\boldsymbol{z}^{\prime}$ and $\overline{\boldsymbol{z}}^{\prime}$ in the DA and the CCA, respectively. Then the integral over $\boldsymbol{k}_{3}$ enforces $\boldsymbol{z}^{\prime}=\overline{\boldsymbol{z}}^{\prime}$ and the longitudinal fractions should be computed as in eq. (4.11), that is,

$$
\theta=1-\frac{k_{1}^{+}}{q^{+}}=1-\frac{x_{1}}{x_{q}}, \quad \xi=\frac{k_{2}^{+}}{q^{+}}=\frac{x_{2}}{x_{q}} .
$$

It is again instructive to display the main simplifications in the colour structure due to the fact that one of the final gluons is not measured $\left(\boldsymbol{z}^{\prime}=\overline{\boldsymbol{z}}^{\prime}\right)$. One finds

$$
\begin{aligned}
& S_{q g g \bar{q} g g}^{(1)}\left(\boldsymbol{x}, \boldsymbol{z}, \boldsymbol{z}^{\prime}, \overline{\boldsymbol{x}}, \overline{\boldsymbol{z}}, \overline{\boldsymbol{z}}^{\prime}=\boldsymbol{z}^{\prime}\right) \simeq S_{q g g \bar{q} g g}^{(3)}\left(\boldsymbol{x}, \boldsymbol{z}, \boldsymbol{z}^{\prime}, \overline{\boldsymbol{x}}, \overline{\boldsymbol{z}}, \overline{\boldsymbol{z}}^{\prime}=\boldsymbol{z}^{\prime}\right) \simeq \mathcal{S}(\boldsymbol{x}, \overline{\boldsymbol{x}}) \mathcal{S}(\overline{\boldsymbol{z}}, \boldsymbol{z}) \mathcal{S}(\boldsymbol{z}, \overline{\boldsymbol{z}}), \\
& S_{q g g \bar{q} g g}^{(2)}\left(\boldsymbol{x}, \boldsymbol{z}, \boldsymbol{z}^{\prime}, \overline{\boldsymbol{x}}, \overline{\boldsymbol{z}}, \overline{\boldsymbol{z}}^{\prime}=\boldsymbol{z}^{\prime}\right) \simeq \frac{1}{2}[\mathcal{S}(\boldsymbol{x}, \overline{\boldsymbol{x}}) \mathcal{S}(\overline{\boldsymbol{z}}, \boldsymbol{z}) \mathcal{S}(\boldsymbol{z}, \overline{\boldsymbol{z}})+\mathcal{S}(\boldsymbol{z}, \overline{\boldsymbol{z}}) \mathcal{Q}(\boldsymbol{x}, \boldsymbol{z}, \overline{\boldsymbol{z}}, \overline{\boldsymbol{x}})] .
\end{aligned}
$$

\section{Soft gluon emissions: recovering the B-JIMWLK evolution}

In this section we shall study the behaviour of the previous results in the limit where one of the two final gluons is very soft, that is, either $\xi \rightarrow 0$ or $\theta-\xi \rightarrow 0$. This is interesting since, in this limit, our general results should reduce to one-step in the B-JIMWLK (or $\mathrm{BK}$ ) evolution of the leading-order dijet cross-section. As we shall see, this expectation is indeed verified and it provides a rather strong test of the correctness of our previous calculation. For more generality, we shall first address the soft gluon limit for the trijet ( $q g g)$ cross-section, before eventually specialising to the (real) NLO corrections to the dijet $(q g)$ cross-section. 


\subsection{Direct emissions by the quark}

We start with the contribution to the cross-section shown in eq. (3.39), where the two gluons are directly emitted by the quark, both in the DA and in the CCA (recall figure 9). When (at least) one of these gluons is soft, there are two types of simplifications.

First one can neglect the recoil of the emitter (here, the quark) at the respective emission vertex, meaning that its transverse coordinate is not modified by the soft emission. With reference to figure 6 , when $\xi \rightarrow 0$ one can approximate $\boldsymbol{y} \simeq \boldsymbol{w} \simeq(1-\theta) \boldsymbol{x}+\theta \boldsymbol{z}^{\prime}$, whereas for $\theta-\xi \rightarrow 0$, one has $\boldsymbol{y} \simeq \boldsymbol{x}$ and $\boldsymbol{w} \simeq(1-\theta) \boldsymbol{x}+\theta \boldsymbol{z}$.

Second, one can simplify the dependence of the kernel (3.40) - which, we recall, encodes information about both the emission vertices and the energy denominators - upon the longitudinal momentum fractions $\theta$ and $\xi$. It is easy to check that the instantaneous piece of the effective vertex $\Xi$ (cf. eq. (3.21)) does not contribute to the would-be singularity in the soft limit. Hence, one can use the expression (3.41) for the square of the effective vertex. This gives the following soft limits:

$$
\begin{aligned}
\Xi_{\lambda_{1} \lambda}^{i j m n}(\theta, \xi) \Xi_{\lambda_{1} \lambda}^{i j p q *}(\theta, \xi) & \longrightarrow \delta^{m p} \delta^{n q} \frac{16 \theta(1-\theta)^{4}\left[1+(1-\theta)^{2}\right]}{\theta-\xi}, \text { when } \theta-\xi \rightarrow 0, \\
& \longrightarrow \delta^{m p} \delta^{n q} \frac{16 \xi\left[1+(1-\theta)^{2}\right]}{\theta}, \text { when } \xi \rightarrow 0 .
\end{aligned}
$$

Concerning the denominator of $\mathcal{K}_{1}$, the limit $\theta-\xi \rightarrow 0$ poses no special difficulty and yields

$$
\mathcal{K}_{1}^{m n p q} \longrightarrow \delta^{m p} \delta^{n q} \frac{16\left[1+(1-\theta)^{2}\right]}{\theta(\theta-\xi) \boldsymbol{Y}^{2} \overline{\boldsymbol{Y}}^{2}} \quad \text { when } \quad \theta-\xi \rightarrow 0 .
$$

The other eikonal limit, namely $\xi \rightarrow 0$, is a bit more subtle, as it does not commute with the equal transverse points limits which define three of the four terms within the square brackets in eq. (3.39). Keeping the dominant terms as $\xi \rightarrow 0$ in each of the respective kernels, one finds

$$
\begin{aligned}
\left.\mathcal{K}_{1}^{m n p q}\right|_{\xi \rightarrow 0} & \longrightarrow 0, \quad \text { when both } \boldsymbol{X}^{\prime} \neq 0 \text { and } \overline{\boldsymbol{X}}^{\prime} \neq 0, \\
& \longrightarrow \delta^{m p} \delta^{n q} \frac{16\left[1+(1-\theta)^{2}\right]}{\theta^{2}(1-\theta) \boldsymbol{Y}^{2}\left(\overline{\boldsymbol{X}}^{\prime}\right)^{2}}, \quad \text { when } \boldsymbol{X}^{\prime}=0 \text {, but } \overline{\boldsymbol{X}}^{\prime} \neq 0, \\
& \longrightarrow \delta^{m p} \delta^{n q} \frac{16\left[1+(1-\theta)^{2}\right]}{\theta^{2}(1-\theta)\left(\boldsymbol{X}^{\prime}\right)^{2} \overline{\boldsymbol{Y}}^{2}}, \quad \text { when } \boldsymbol{X}^{\prime} \neq 0 \text {, but } \overline{\boldsymbol{X}}^{\prime}=0, \\
& \longrightarrow \delta^{m p} \delta^{n q} \frac{16\left[1+(1-\theta)^{2}\right]}{\xi \theta \boldsymbol{Y}^{2} \overline{\boldsymbol{Y}}^{2}}, \quad \text { when } \boldsymbol{X}^{\prime}=\overline{\boldsymbol{X}}^{\prime}=0,
\end{aligned}
$$

These expressions exhibit single poles at $\theta=\xi$ and respectively $\xi=0$, which are the expected infrared singularities associated with the bremsstrahlung of very soft gluons. What is however quite remarkable and also important for what follows, is the special way how these singularities appear within the structure of the cross-section (3.39): (i) the pole at $\theta=\xi$ is common to all the four terms in eq. (3.39) and, moreover, the respective kernels become degenerate in this limit; (ii) the pole at $\xi=0$ refers only to the last term 

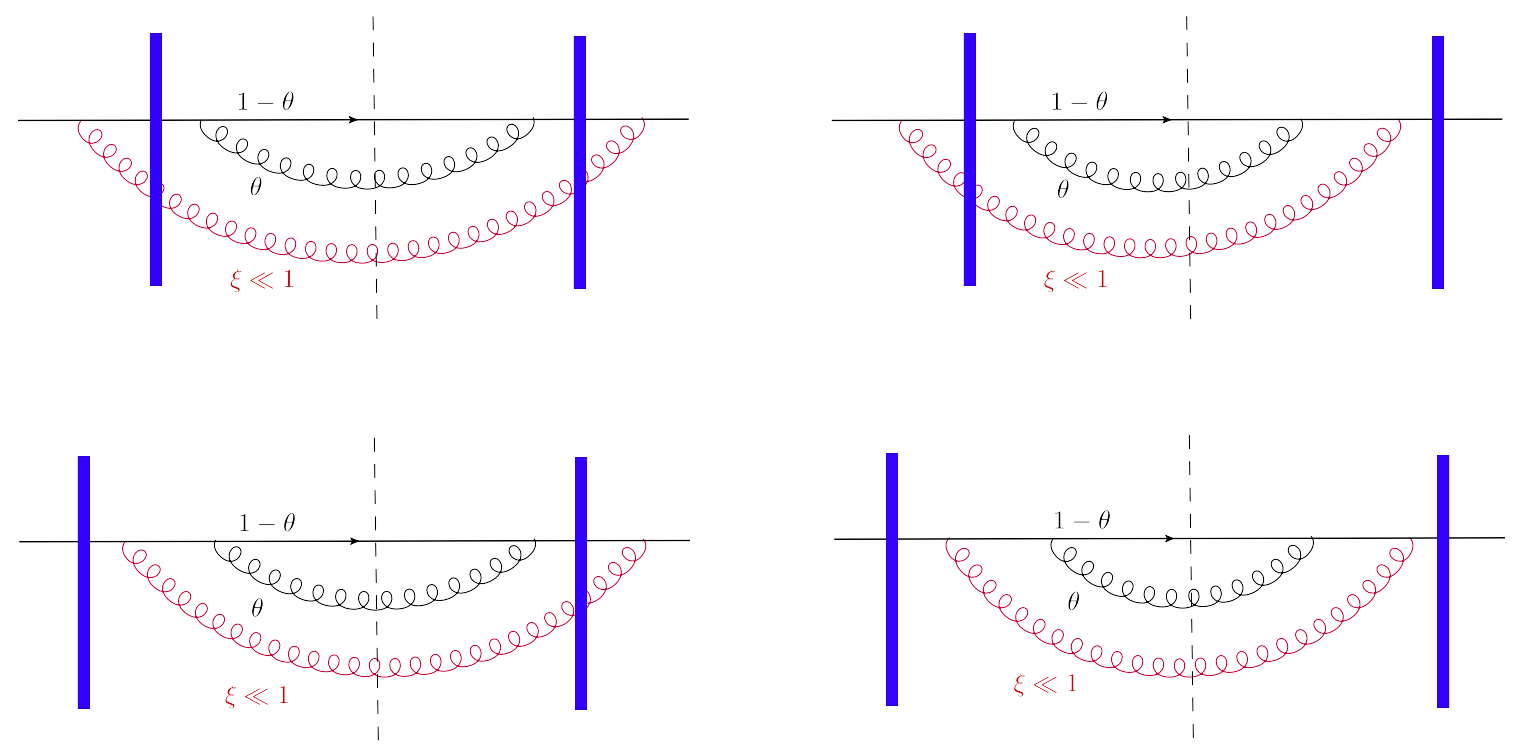

Figure 12. The four diagrams which dominate the cross-section (3.39) in the eikonal limit $\xi \rightarrow 0$ in which the first emitted gluon (shown in red) is soft.
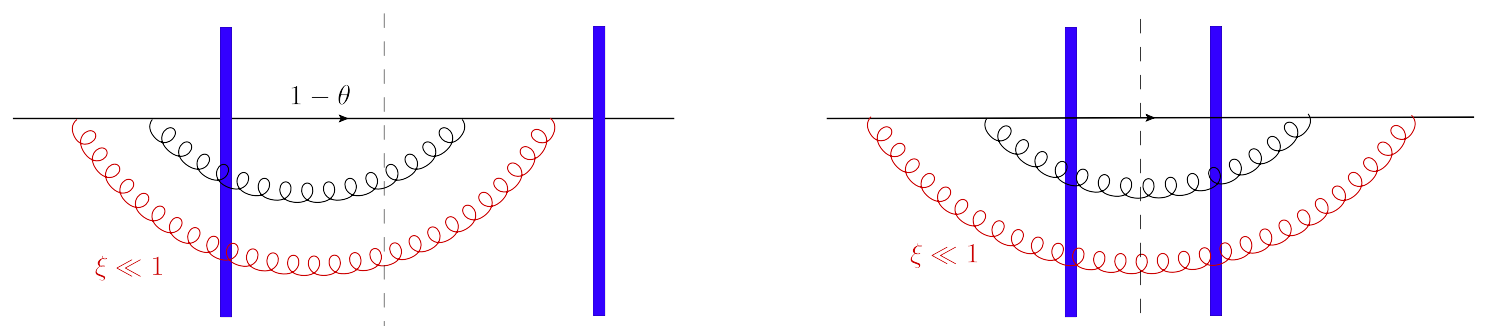

Figure 13. Diagrams which contribute to the $q g g$ cross-section (3.39) in general, but which do not survive in the eikonal (soft) limit for the gluon with longitudinal momentum fraction $\xi$ (shown in red). These graphs do not contribute to the JIMWLK evolution of any of the $S$-matrices which enter the LO cross-section (2.10).

in eq. (3.39), as obtained via the double replacement $\left(\boldsymbol{x}, \boldsymbol{z}^{\prime} \rightarrow \boldsymbol{y} \& \overline{\boldsymbol{x}}, \overline{\boldsymbol{z}}^{\prime} \rightarrow \overline{\boldsymbol{y}}\right)$. These properties are essential in order to recover the JIMWLK evolution, as we now explain.

The limit $\xi \rightarrow 0$ corresponds to the case where the soft gluon is the first one to be emitted (see figure 6). Property (ii) above tells us that the dominant graphs in this limit are the four graphs shown in figure 12, where the interaction with the shockwave occurs either in the initial, or in the intermediate, state (the soft gluon is drawn in red). Notice that this is only a subset of all the possible topologies which contribute to the cross-section (3.39) in general: 4 out of 9 . The missing diagrams are those which describe initial-state evolution (equivalently, final-state scattering) in either the DA, or in the CCA, or in both; that is, both gluon emissions occur prior to the scattering off the shockwave. (Two of the excluded diagrams are illustrated in figure 13.) This is in agreement with the fact that a soft gluon has a short formation time, hence it must be emitted as close as possible to the nuclear target in order to contribute to the scattering. 
The graphs shown in figure 12 have the right structure to describe one step in the JIMWLK evolution of the LO dijet cross-section: they precisely encode the BK evolution of the last piece in eq. (2.10) - the dipole $S$-matrix $\mathcal{S}(\boldsymbol{w}, \overline{\boldsymbol{w}})$ - , as we now demonstrate. Let us use the momentum assignments shown in figure 9, i.e. $k_{2}^{+}=\xi q^{+}$and $k_{3}^{+}=(\theta-\xi) q^{+}$, with $\xi \rightarrow 0$. In this limit, eq. (3.39) simplifies to

$$
\begin{aligned}
& \left.\frac{d \sigma_{(1)}^{q A \rightarrow q g g+X}}{d k_{1}^{+} d^{2} \boldsymbol{k}_{1} d k_{2}^{+} d^{2} \boldsymbol{k}_{2} d k_{3}^{+} d^{2} \boldsymbol{k}_{3}}\right|_{\xi \rightarrow 0}=\frac{8 \alpha_{s}^{2} C_{F}^{2}}{(2 \pi)^{10}} \frac{1+(1-\theta)^{2}}{\xi \theta\left(q^{+}\right)^{2}} \delta\left(q^{+}-k_{1}^{+}-k_{3}^{+}\right) \\
& \times \int_{\overline{\boldsymbol{x}}, \overline{\boldsymbol{z}}^{\prime}, \overline{\boldsymbol{z}}^{\prime}, \boldsymbol{x}, \boldsymbol{z}, \boldsymbol{z}^{\prime}} e^{-i \boldsymbol{k}_{1} \cdot(\boldsymbol{x}-\overline{\boldsymbol{x}})-i \boldsymbol{k}_{2} \cdot(\boldsymbol{z}-\overline{\boldsymbol{z}})-i \boldsymbol{k}_{3} \cdot\left(\boldsymbol{z}^{\prime}-\overline{\boldsymbol{z}}^{\prime}\right)} \frac{(\boldsymbol{Y} \cdot \overline{\boldsymbol{Y}})\left(\boldsymbol{X}^{\prime} \cdot \overline{\boldsymbol{X}}^{\prime}\right)}{\boldsymbol{Y}^{2} \overline{\boldsymbol{Y}}^{2}\left(\boldsymbol{X}^{\prime}\right)^{2}\left(\overline{\boldsymbol{X}}^{\prime}\right)^{2}} \\
& \times[\mathcal{Q}(\boldsymbol{w}, \boldsymbol{z}, \overline{\boldsymbol{z}}, \overline{\boldsymbol{w}}) \mathcal{S}(\boldsymbol{z}, \overline{\boldsymbol{z}})-\mathcal{S}(\boldsymbol{w}, \boldsymbol{z}) \mathcal{S}(\boldsymbol{z}, \overline{\boldsymbol{w}})-\mathcal{S}(\boldsymbol{w}, \overline{\boldsymbol{z}}) \mathcal{S}(\overline{\boldsymbol{z}}, \overline{\boldsymbol{w}})+\mathcal{S}(\boldsymbol{w}, \overline{\boldsymbol{w}})]
\end{aligned}
$$

where we have used $\boldsymbol{w} \simeq \boldsymbol{y} \simeq(1-\theta) \boldsymbol{x}+\theta \boldsymbol{z}^{\prime}$ and we recall that $\boldsymbol{Y}=\boldsymbol{y}-\boldsymbol{z} \simeq \boldsymbol{w}-\boldsymbol{z}$ and $\boldsymbol{X}^{\prime}=\boldsymbol{x}-\boldsymbol{z}^{\prime}$ (and similarly for the respective coordinates with a bar). Also, we have ignored the soft momentum $k_{2}^{+}$in the $\delta$-function for longitudinal momentum conservation. This is in the spirit of the soft gluon approximation to the high-energy evolution, which effectively violates energy conservation, albeit only marginally (by ignoring the soft gluons in the energy balance).

As expected, eq. (5.4) is the same as the result of acting with the "production" version of the JIMWLK Hamiltonian [81-83] on the last term (the dipole $\mathcal{S}(\boldsymbol{w}, \overline{\boldsymbol{w}})$ ) in the LO $q g$ cross-section (2.10). The "production" Hamiltonian is a generalised version of the JIMWLK Hamiltonian which, when acting on the cross-section for particle production in dilute-dense ( $p A$ or $e A$ ) collisions, leads to the emission of an additional, soft, gluon, which is measured in the final state. If on the other hand this soft gluon is not measured, i.e. if one integrates out its kinematics, then one generates the "real" piece of the standard JIMWLK evolution. By integrating eq. (5.4) over $k_{2}^{+}=\xi q^{+}$and $\boldsymbol{k}_{2}$, one finds (with $2 \alpha_{s} C_{F} \simeq \alpha_{s} N_{c} \equiv \pi \bar{\alpha}_{s}$ at large $N_{c}$ )

$$
\begin{aligned}
\frac{d \sigma_{\mathrm{NLO}, 1}^{q A \rightarrow q g+X}}{d k_{1}^{+} d^{2} \boldsymbol{k}_{1} d k_{3}^{+} d^{2} \boldsymbol{k}_{3}} \simeq & \frac{\bar{\alpha}_{s}}{(2 \pi)^{5}} \frac{1+(1-\theta)^{2}}{2 \theta q^{+}} \delta\left(q^{+}-k_{1}^{+}-k_{3}^{+}\right) \\
& \times \int_{\overline{\boldsymbol{x}}_{\overline{\boldsymbol{z}}}^{\prime}, \boldsymbol{x}, \boldsymbol{z}^{\prime}} e^{-i \boldsymbol{k}_{1} \cdot(\boldsymbol{x}-\overline{\boldsymbol{x}})-i \boldsymbol{k}_{3} \cdot\left(\boldsymbol{z}^{\prime}-\overline{\boldsymbol{z}}^{\prime}\right)} \frac{\left(\boldsymbol{x}-\boldsymbol{z}^{\prime}\right) \cdot\left(\overline{\boldsymbol{x}}-\overline{\boldsymbol{z}}^{\prime}\right)}{\left(\boldsymbol{x}-\boldsymbol{z}^{\prime}\right)^{2}\left(\overline{\boldsymbol{x}}-\overline{\boldsymbol{z}}^{\prime}\right)^{2}} \\
& \times \frac{\bar{\alpha}_{s}}{2 \pi} \int_{0}^{1} \frac{d \xi}{\xi} \int_{\boldsymbol{z}} \frac{2(\boldsymbol{w}-\boldsymbol{z}) \cdot(\overline{\boldsymbol{w}}-\boldsymbol{z})}{(\boldsymbol{w}-\boldsymbol{z})^{2}(\overline{\boldsymbol{w}}-\boldsymbol{z})^{2}}[\mathcal{S}(\boldsymbol{w}, \overline{\boldsymbol{w}})-\mathcal{S}(\boldsymbol{w}, \boldsymbol{z}) \mathcal{S}(\boldsymbol{z}, \overline{\boldsymbol{w}})]
\end{aligned}
$$

Notice the simplifications in the colour structure due to the fact that the integral over $\boldsymbol{k}_{2}$ has identified the coordinates $\boldsymbol{z}$ and $\overline{\boldsymbol{z}}$ of the unmeasured gluon: $\boldsymbol{z}=\overline{\boldsymbol{z}}$.

The expression in the third line of eq. (5.5) is recognised as the "real" part of the BK equation for the evolution of the dipole $\mathcal{S}(\boldsymbol{w}, \overline{\boldsymbol{w}})$. More precisely, it exhibits the integral version of the BK equation, that is, its formal solution as obtained by integrating the r.h.s. of that equation over $\xi$. This integral exhibits a logarithmic divergence at its lower limit $(\xi=0)$. In reality, this divergence is cut off by the condition of energy conservation. Indeed, 

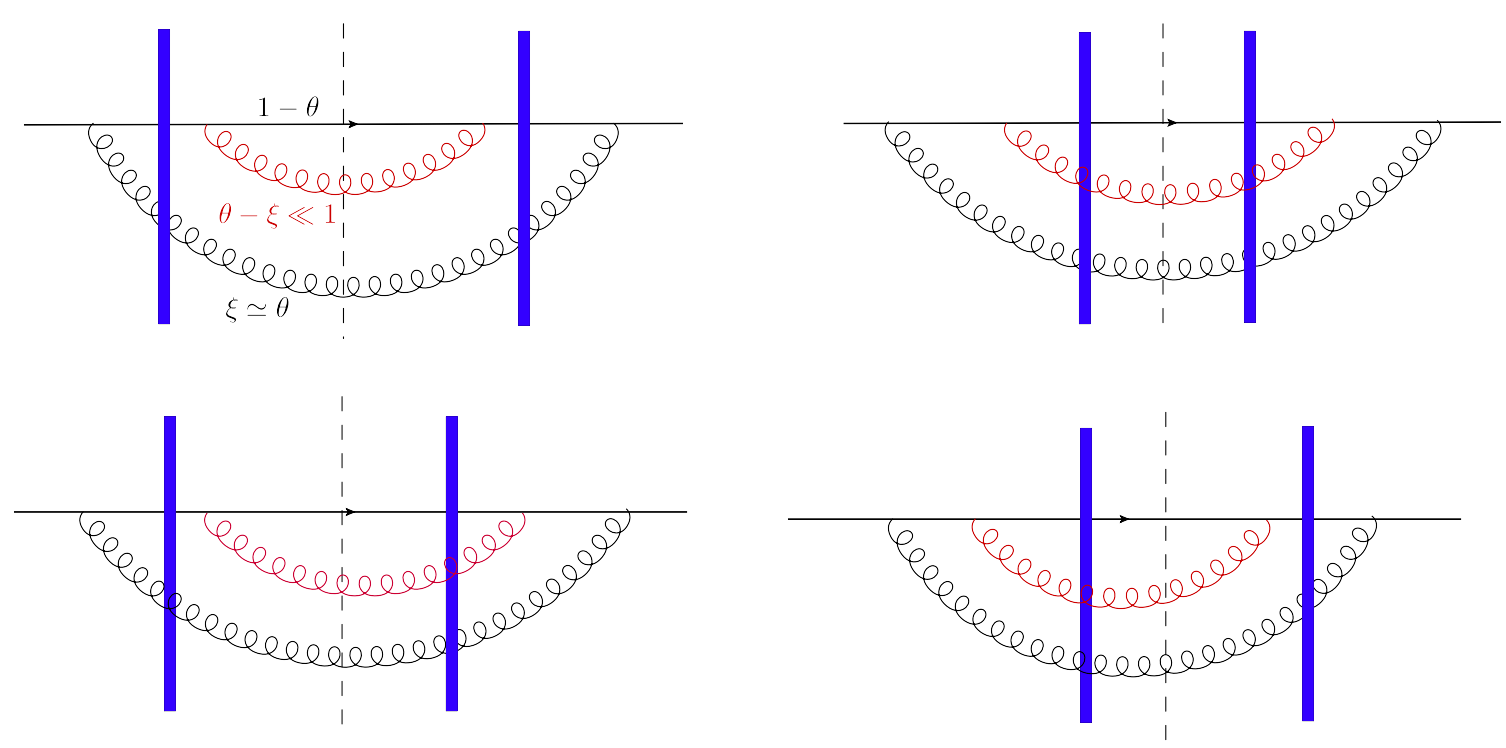

Figure 14. The four diagrams contributing to the cross-section (3.39) which survive in the eikonal limit $\theta-\xi \rightarrow 0$ in which the second emitted gluon (shown in red) is soft.

this integral can be equivalently rewritten as $\int(d \xi / \xi)=\int\left(d x_{2} / x_{2}\right)$, with $x_{2}=k_{2}^{+} / Q^{+}$; then eq. (3.37) together with the constraint $x_{g} \leq 1$ clearly implies a lower-limit on $x_{2}$, hence on $\xi$.

This discussion also shows that the soft $(\xi \ll 1)$ part of the integral over the unmeasured gluon contributes to the high-energy (B-JIMWLK) evolution of the LO dijet cross-section, so it is only the remaining contribution at large $\xi \sim 1$ which should be viewed as a genuine NLO correction to the "hard impact factor", i.e. to the partonic crosssection for the $q A \rightarrow q g+X$ process. That said, it turns out that the separation of the full integral over $\xi$ between a "soft part" describing LO evolution and a "hard part" describing NLO corrections to the impact factor is generally subtle, due to the fact that the various $S$-matrices in eq. (5.5) do implicitly depend upon $\xi$. (Indeed, their rapidity evolution scale is $x_{g}$, which is a function of $x_{2}$, hence of $\xi$, as shown in eq. (3.37).) This dependence is essential, as argued in refs. $[43,47]$ : attempts to separate the "soft" evolution from the "hard" NLO impact factor which ignore this evolution may lead into troubles, like negative values for the NLO cross-section, as observed in the context of single inclusive particle production in both $p A[21,31,46]$ and $e A$ collisions [48]. A detailed discussion of such issues in the context of dijet production goes well beyond the purposes of this paper. But one should keep them in mind when trying to actually estimate the NLO corrections (say, for the purposes of the phenomenology).

Consider similarly the eikonal limit for the second emitted gluon, that with longitudinal momentum $k_{3}^{+}=(\theta-\xi) q^{+}$. Eq. (5.2) implies that, when this gluon is soft $(\theta-\xi \ll 1)$, all the four terms within the squared brackets in eq. (3.39) are multiplied by the same kernel, hence the respective colour structures can be simply added to each other. Remarkably, 12 of the 16 partonic $S$-matrices which are a priori contained in these structures mutually 

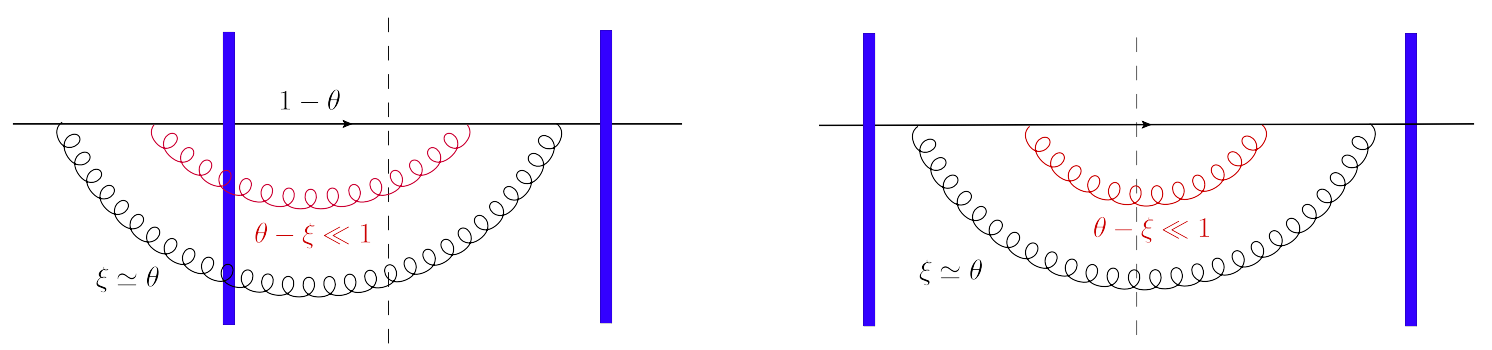

Figure 15. Diagrams which contribute to the $q g g$ cross-section (3.39) in general, but which do not survive in the eikonal (soft) limit for the gluon with longitudinal momentum fraction $\theta-\xi$ (shown in red).

cancel in their sum, and we are left with

$$
\begin{aligned}
& \left.\frac{d \sigma_{(1)}^{q A \rightarrow q g g+X}}{d k_{1}^{+} d^{2} \boldsymbol{k}_{1} d k_{2}^{+} d^{2} \boldsymbol{k}_{2} d k_{3}^{+} d^{2} \boldsymbol{k}_{3}}\right|_{\theta-\xi \rightarrow 0}=\frac{8 \alpha_{s}^{2} C_{F}^{2}}{(2 \pi)^{10}} \frac{1+(1-\theta)^{2}}{(\theta-\xi) \theta\left(q^{+}\right)^{2}} \delta\left(q^{+}-k_{1}^{+}-k_{2}^{+}\right) \\
& \times \int_{\overline{\boldsymbol{x}}, \overline{\boldsymbol{z}}, \overline{\boldsymbol{z}}^{\prime}, \boldsymbol{x}, \boldsymbol{z}, \boldsymbol{z}^{\prime}} e^{-i \boldsymbol{k}_{1} \cdot(\boldsymbol{x}-\overline{\boldsymbol{x}})-i \boldsymbol{k}_{2} \cdot(\boldsymbol{z}-\overline{\boldsymbol{z}})-i \boldsymbol{k}_{3} \cdot\left(\boldsymbol{z}^{\prime}-\overline{\boldsymbol{z}}^{\prime}\right)} \frac{(\boldsymbol{X} \cdot \overline{\boldsymbol{X}})\left(\boldsymbol{X}^{\prime} \cdot \overline{\boldsymbol{X}}^{\prime}\right)}{\boldsymbol{X}^{2} \overline{\boldsymbol{X}}^{2}\left(\boldsymbol{X}^{\prime}\right)^{2}\left(\overline{\boldsymbol{X}}^{\prime}\right)^{2}} \\
& \times\left[\mathcal{Q}\left(\boldsymbol{x}, \boldsymbol{z}^{\prime}, \overline{\boldsymbol{z}}^{\prime}, \overline{\boldsymbol{x}}\right) \mathcal{Q}\left(\boldsymbol{z}^{\prime}, \boldsymbol{z}, \overline{\boldsymbol{z}}, \overline{\boldsymbol{z}}^{\prime}\right)-\mathcal{S}\left(\overline{\boldsymbol{z}}^{\prime}, \overline{\boldsymbol{x}}\right) \mathcal{Q}\left(\boldsymbol{x}, \boldsymbol{z}, \overline{\boldsymbol{z}}, \overline{\boldsymbol{z}}^{\prime}\right)\right. \\
& \left.\quad-\mathcal{S}\left(\boldsymbol{x}, \boldsymbol{z}^{\prime}\right) \mathcal{Q}\left(\boldsymbol{z}^{\prime}, \boldsymbol{z}, \overline{\boldsymbol{z}}, \overline{\boldsymbol{x}}\right)+\mathcal{Q}(\boldsymbol{x}, \boldsymbol{z}, \overline{\boldsymbol{z}}, \overline{\boldsymbol{x}})\right] \mathcal{S}(\boldsymbol{z}, \overline{\boldsymbol{z}})
\end{aligned}
$$

where $\boldsymbol{X}=\boldsymbol{x}-\boldsymbol{z}$ and $\boldsymbol{X}^{\prime}=\boldsymbol{x}-\boldsymbol{z}^{\prime}$ (we have used $\boldsymbol{y} \simeq \boldsymbol{x}$ ). The four surviving $S$-matrices correspond to the situations where the shockwave is inserted either in the final qgg state, or in the intermediate $q g$ state (see figure 14), so that at least one gluon participates in the scattering (together with the quark, of course). For more clarity, we also show in figure 15 two graphs whose contributions have cancelled in the overall sum.

The four surviving graphs in figure 14 follow the same pattern as that illustrated in figure 12: the soft gluon is the last (first) one to be emitted prior to (after) the shockwave. In this case too, they describe the JIMWLK evolution of one of the $S$-matrices from eq. (2.10) - here, the colour quadrupole which enters in the structure of the first term there, cf. eq. (2.14) and (2.17). Specifically, these are the four diagrams describing the evolution of the quadrupole $\mathcal{Q}(\boldsymbol{x}, \boldsymbol{z}, \overline{\boldsymbol{z}}, \overline{\boldsymbol{x}})$ via the emission of a soft gluon which is emitted at $\boldsymbol{x}$ in the DA and reabsorbed at $\overline{\boldsymbol{x}}$ in the CCA. One can check (e.g. by comparing to eq. (A.5) in ref. [83]) that the various factors in eq. (5.6) are precisely as needed in order to describe this particular evolution. ${ }^{11}$ Of course, the JIMWLK evolution of the quadrupole also involves other diagrams, in which the soft gluon is emitted/absorbed by some other pair of external legs $[71-73,83]$. These additional diagrams will be generated by the remaining contributions to the $q g g$ cross-section, whose soft limit will be explored in the next two subsections.

\footnotetext{
${ }^{11}$ In the case where the soft gluon $k_{3}$ is not measured (which amounts to identifying $\boldsymbol{z}^{\prime}=\boldsymbol{z}$ ), we precisely recover the relevant "real" terms from the JIMWLK equation for the high-energy evolution of the quadrupole $S$-matrix [71-73].
} 


\subsection{Direct contributions involving the 3 -gluon vertex}

The discussion of the soft gluon limit for the second contribution to the $q g g$ cross-section, eq. (3.46), is somewhat simpler, due to the manifest symmetry of this contribution under the exchange of the two final gluons. It is therefore enough to consider only one of the two eikonal limits aforementioned, say $\xi \rightarrow 0$. In this limit, the triple-gluon vertex in eq. (3.24) can be approximated as

$$
\Gamma^{n l i j}(\theta, \xi \rightarrow 0) \simeq-\frac{1}{\xi} \delta_{n i} \delta_{l j} .
$$

It is then easily to see, by using the expressions (3.23) for the relevant effective vertex and (3.47) for the kernel, that the latter admits the same limit for all the four terms within the square brackets in eq. (3.46), that is,

$$
\left.\mathcal{K}_{2}^{m n p q}\right|_{\xi \rightarrow 0}=\frac{4\left[1+(1-\theta)^{2}\right] \delta^{m p} \delta^{n q}}{\theta \xi \boldsymbol{R}^{2} \overline{\boldsymbol{R}}^{2}} .
$$

This expression shows the expected soft singularity: a single pole at $\xi=0$. As before, this degeneracy of the four kernels implies that only four topologies survive in the final result (among the 9 which were originally present in the r.h.s. of eq. (3.46)): those in which the shockwave is inserted in either the final $q g g$ state, or in the intermediate $q g$ state. The surviving configurations are shown in figure 16. At large $N_{c}$, each of the $S$-matrices associated with these four graphs is in turn built with two colour structures, corresponding to permutations of the two gluons, as shown e.g. in eq. (3.50). Still for $\xi \rightarrow 0$, one can also identify $\boldsymbol{z}^{\prime}=\boldsymbol{y}$ and $\overline{\boldsymbol{z}}^{\prime}=\overline{\boldsymbol{y}}$ in the arguments of the surviving $S$-matrices.

We are thus led to the following result (we use the conventions in figure 10, i.e. the $k_{2}^{+}=\xi q^{+}$and $\left.k_{3}^{+}=(\theta-\xi) q^{+}\right)$:

$$
\begin{aligned}
&\left.\frac{d \sigma_{(2)}^{q A \rightarrow q g g+X}}{d k_{1}^{+} d^{2} \boldsymbol{k}_{1} d k_{2}^{+} d^{2} \boldsymbol{k}_{2} d k_{3}^{+} d^{2} \boldsymbol{k}_{3}}\right|_{\xi \rightarrow 0}=\frac{4 \alpha_{s}^{2} C_{F} N_{c}}{(2 \pi)^{10}} \frac{1+(1-\theta)^{2}}{\xi \theta\left(q^{+}\right)^{2}} \delta\left(q^{+}-k_{1}^{+}-k_{3}^{+}\right) \\
& \times \int_{\overline{\boldsymbol{x}}, \overline{\boldsymbol{z}}, \overline{\boldsymbol{y}}, \boldsymbol{x}, \boldsymbol{z}, \boldsymbol{y}} e^{-i \boldsymbol{k}_{1} \cdot(\boldsymbol{x}-\overline{\boldsymbol{x}})-i \boldsymbol{k}_{2} \cdot(\boldsymbol{z}-\overline{\boldsymbol{z}})-i \boldsymbol{k}_{3} \cdot(\boldsymbol{y}-\overline{\boldsymbol{y}})} \frac{(\boldsymbol{R} \cdot \overline{\boldsymbol{R}})(\boldsymbol{Z} \cdot \overline{\boldsymbol{Z}})}{\boldsymbol{R}^{2} \overline{\boldsymbol{R}}^{2} \boldsymbol{Z}^{2} \overline{\boldsymbol{Z}}^{2}} \\
& \times\{[\mathcal{Q}(\boldsymbol{x}, \boldsymbol{z}, \overline{\boldsymbol{z}}, \overline{\boldsymbol{x}}) \mathcal{Q}(\boldsymbol{z}, \boldsymbol{y}, \overline{\boldsymbol{y}}, \overline{\boldsymbol{z}})-\mathcal{Q}(\boldsymbol{x}, \boldsymbol{y}, \overline{\boldsymbol{z}}, \overline{\boldsymbol{x}}) \mathcal{S}(\overline{\boldsymbol{y}}, \overline{\boldsymbol{z}}) \\
&-\mathcal{Q}(\boldsymbol{x}, \boldsymbol{z}, \overline{\boldsymbol{y}}, \overline{\boldsymbol{x}}) \mathcal{S}(\boldsymbol{z}, \boldsymbol{y})+\mathcal{Q}(\boldsymbol{x}, \boldsymbol{y}, \overline{\boldsymbol{y}}, \overline{\boldsymbol{x}})] \mathcal{S}(\boldsymbol{y}, \overline{\boldsymbol{y}}) \\
&+[\mathcal{Q}(\boldsymbol{y}, \boldsymbol{z}, \overline{\boldsymbol{z}}, \overline{\boldsymbol{y}}) \mathcal{S}(\boldsymbol{z}, \overline{\boldsymbol{z}})-\mathcal{S}(\overline{\boldsymbol{z}}, \overline{\boldsymbol{y}}) \mathcal{S}(\boldsymbol{y}, \overline{\boldsymbol{z}}) \\
&-\mathcal{S}(\boldsymbol{y}, \boldsymbol{z}) \mathcal{S}(\boldsymbol{z}, \overline{\boldsymbol{y}})+\mathcal{S}(\boldsymbol{y}, \overline{\boldsymbol{y}})] \mathcal{Q}(\boldsymbol{x}, \boldsymbol{y}, \overline{\boldsymbol{y}}, \overline{\boldsymbol{x}})\}
\end{aligned}
$$

where $\boldsymbol{Z}=\boldsymbol{z}-\boldsymbol{y}, \boldsymbol{R}=\boldsymbol{x}-\boldsymbol{y}$ and similarly for the coordinates with a bar. Both the first term within the first square bracket and the first term within the second square bracket are generated by the first graph in figure 16, via its decomposition at large $N_{c}$, which is graphically illustrated in figure 17. This figure also makes clear that the four terms within the first square bracket describe the emission of a soft gluon from the quadrupole 

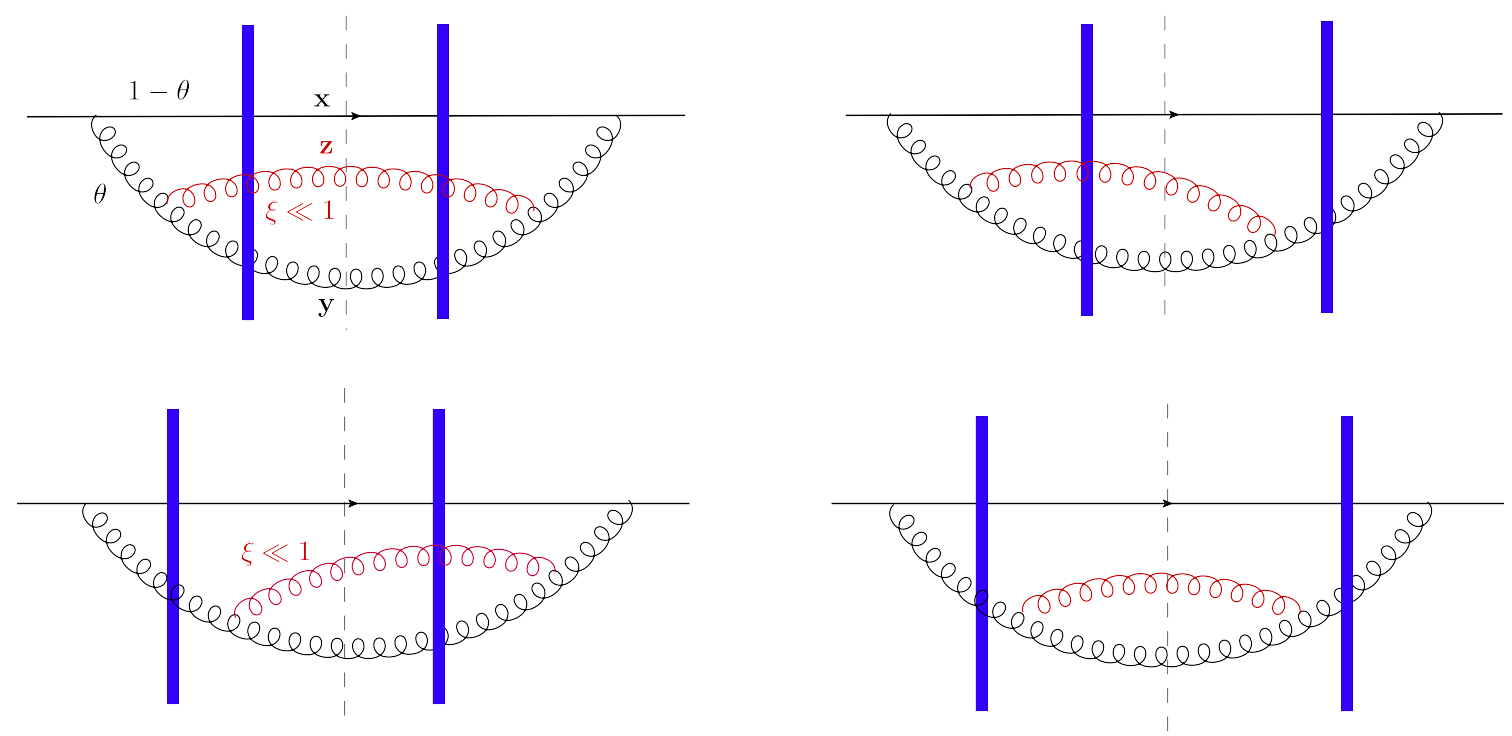

Figure 16. The four diagrams contributing to the cross-section (3.46) in the eikonal limit $\xi \rightarrow 0$ for one of the two emitted gluons (the one shown in red).

$\mathcal{Q}(\boldsymbol{x}, \boldsymbol{y}, \overline{\boldsymbol{y}}, \overline{\boldsymbol{x}})$, such that the soft gluon is emitted at $\boldsymbol{y}$ in the DA and at $\overline{\boldsymbol{y}}$ in the CCA. Similarly, the four terms within the second square bracket describe the emission of a soft gluon from the dipole $\mathcal{S}(\boldsymbol{y}, \overline{\boldsymbol{y}})$.

The result (5.9) takes a more familiar form in the case when the soft gluon $k_{2}$ is not measured, meaning that one can integrate over its kinematics (which in particular allows us to identify $\boldsymbol{z}=\overline{\boldsymbol{z}}$ ).

$$
\begin{aligned}
\frac{d \sigma_{\mathrm{NLO}, 2}^{q A \rightarrow q g+X}}{d k_{1}^{+} d^{2} \boldsymbol{k}_{1} d k_{3}^{+} d^{2} \boldsymbol{k}_{3}} \simeq & \frac{\bar{\alpha}_{s}}{(2 \pi)^{5}} \frac{1+(1-\theta)^{2}}{2 \theta q^{+}} \delta\left(q^{+}-k_{1}^{+}-k_{3}^{+}\right) \\
& \times \int_{\overline{\boldsymbol{x}}, \overline{\boldsymbol{y}}, \boldsymbol{x}, \boldsymbol{y}} e^{-i \boldsymbol{k}_{1} \cdot(\boldsymbol{x}-\overline{\boldsymbol{x}})-i \boldsymbol{k}_{3} \cdot(\boldsymbol{y}-\overline{\boldsymbol{y}})} \frac{(\boldsymbol{x}-\boldsymbol{y}) \cdot(\overline{\boldsymbol{x}}-\overline{\boldsymbol{y}})}{(\boldsymbol{x}-\boldsymbol{y})^{2}(\overline{\boldsymbol{x}}-\overline{\boldsymbol{y}})^{2}} \\
& \times \frac{\bar{\alpha}_{s}}{2 \pi} \int_{0}^{1} \frac{d \xi}{\xi} \int_{\boldsymbol{z}} \frac{(\boldsymbol{y}-\boldsymbol{z}) \cdot(\overline{\boldsymbol{y}}-\boldsymbol{z})}{(\boldsymbol{y}-\boldsymbol{z})^{2}(\overline{\boldsymbol{y}}-\boldsymbol{z})^{2}} \\
\times & \{[\mathcal{S}(\boldsymbol{x}, \overline{\boldsymbol{x}}) \mathcal{S}(\boldsymbol{y}, \overline{\boldsymbol{y}})-\mathcal{Q}(\boldsymbol{x}, \boldsymbol{y}, \boldsymbol{z}, \overline{\boldsymbol{x}}) \mathcal{S}(\overline{\boldsymbol{y}}, \boldsymbol{z}) \\
& \quad-\mathcal{Q}(\boldsymbol{x}, \boldsymbol{z}, \overline{\boldsymbol{y}}, \overline{\boldsymbol{x}}) \mathcal{S}(\boldsymbol{z}, \boldsymbol{y})+\mathcal{Q}(\boldsymbol{x}, \boldsymbol{y}, \overline{\boldsymbol{y}}, \overline{\boldsymbol{x}})] \mathcal{S}(\boldsymbol{y}, \overline{\boldsymbol{y}}) \\
& +2[\mathcal{S}(\boldsymbol{y}, \overline{\boldsymbol{y}})-\mathcal{S}(\boldsymbol{y}, \boldsymbol{z}) \mathcal{S}(\boldsymbol{z}, \overline{\boldsymbol{y}})] \mathcal{Q}(\boldsymbol{x}, \boldsymbol{y}, \overline{\boldsymbol{y}}, \overline{\boldsymbol{x}})\}
\end{aligned}
$$

This result is recognised as a piece of the JIMWLK evolution of the first term $S_{q g \bar{q} g}(\boldsymbol{x}, \boldsymbol{y}, \overline{\boldsymbol{x}}, \overline{\boldsymbol{y}}) \simeq \mathcal{Q}(\boldsymbol{x}, \boldsymbol{y}, \overline{\boldsymbol{y}}, \overline{\boldsymbol{x}}) \mathcal{S}(\boldsymbol{y}, \overline{\boldsymbol{y}})$ in the expression (2.17) or the LO cross-section. More precisely, we have the real part of the BK equation for the dipole $\mathcal{S}(\boldsymbol{y}, \overline{\boldsymbol{y}})$ together with the relevant part of the real part of the JIMWLK equation for the quadrupole $\mathcal{Q}(\boldsymbol{x}, \boldsymbol{y}, \overline{\boldsymbol{y}}, \overline{\boldsymbol{x}})$. To complete the (real terms in the) evolution of the latter, we also need the interference contribution in eq. (3.52). This will be discussed in the next section. 

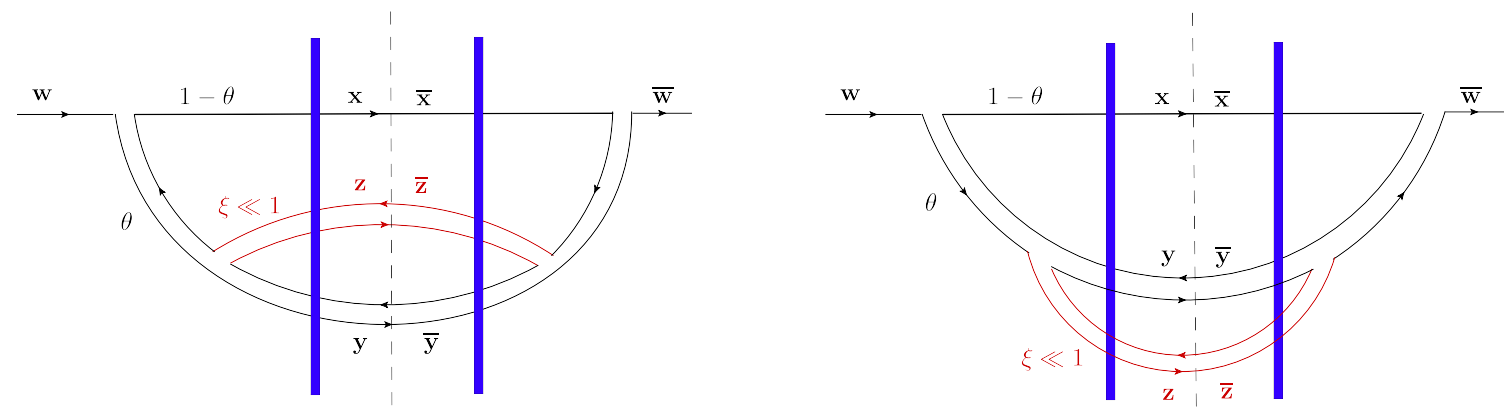

Figure 17. A redrawing of the first graph in figure 16 valid at large $N_{c}$ which exhibits the 2 colour structures hidden in this graph: each gluon is replaced by a double line denoting a quark-antiquark pair of zero transverse size. These two large- $N_{c}$ graphs correspond to the first terms in the first and, respectively, second squared bracket in eq. (5.9).

\subsection{Interference graphs}

We finally consider the eikonal limits for the contributions to the $q g g$ cross-section associated with interference graphs, cf. eq. (3.52) and figure 11. The relevant limits for the product of effective vertices in the numerator of eq. (3.53) read as follows:

$$
\begin{aligned}
\Xi_{\lambda_{1} \lambda}^{i j m n}(\theta, \xi) \Pi_{\lambda_{1} \lambda}^{i j p q *}(\theta, \xi) & \longrightarrow-\delta^{m p} \delta^{n q} \frac{8 \theta^{2}(1-\theta)^{3}\left[1+(1-\theta)^{2}\right]}{\theta-\xi}, \quad \text { when } \theta-\xi \rightarrow 0, \\
& \longrightarrow-8 \delta^{m q} \delta^{n p} \theta(1-\theta)\left[1+(1-\theta)^{2}\right], \quad \text { when } \quad \xi \rightarrow 0 .
\end{aligned}
$$

When $\theta-\xi \rightarrow 0$, all the four kernels in eq. (3.52) take the same form, which reads

$$
\mathcal{K}_{3}^{m n p q} \longrightarrow-\delta^{m p} \delta^{n q} \frac{8\left[1+(1-\theta)^{2}\right]}{\theta(\theta-\xi) \boldsymbol{Y}^{2} \overline{\boldsymbol{R}}^{2}} \quad \text { when } \quad \theta-\xi \rightarrow 0 .
$$

Once again, this means that only four topologies survive in the cross-section in that limit - those in which at least one of the final gluons interacts with the shockwave and which are illustrated in figure 18. Still for $\theta-\xi \rightarrow 0$, we can also approximate the transverse coordinates as $\boldsymbol{y} \simeq \boldsymbol{x}$ and $\overline{\boldsymbol{y}} \simeq \overline{\boldsymbol{z}}$.

Concerning the limit $\xi \rightarrow 0$, we need to distinguish between two cases, depending upon the fact that $\boldsymbol{X}^{\prime}=\boldsymbol{x}-\boldsymbol{z}^{\prime}$ vanishes, or not:

$$
\begin{aligned}
\left.\mathcal{K}_{3}^{m n p q}\right|_{\xi \rightarrow 0} & \longrightarrow-\delta^{m q} \delta^{n p} \frac{8\left[1+(1-\theta)^{2}\right]}{\theta^{2}(1-\theta)\left(\boldsymbol{X}^{\prime}\right)^{2} \overline{\boldsymbol{R}}^{2}}, \quad \text { when } \boldsymbol{X}^{\prime} \neq 0, \\
& \longrightarrow-\delta^{m q} \delta^{n p} \frac{8\left[1+(1-\theta)^{2}\right]}{\xi \theta \boldsymbol{Y}^{2} \overline{\boldsymbol{R}}^{2}}, \quad \text { when } \boldsymbol{X}^{\prime}=0
\end{aligned}
$$

Clearly, the soft singularity at $\xi=0$ is present only in the case where $\boldsymbol{X}^{\prime}=0$. i.e. for the two terms within the square brackets in eq. (3.52) where the coordinates $\boldsymbol{x}$ and $\boldsymbol{z}^{\prime}$ get replaced by $\boldsymbol{y}$. The eight $S$-matrices within the structure of these two terms can now be simply added to each other (since they are all multiplied by the same kernel) and it is easy to see that only four of them survive in the final result: those illustrated in figure 19. Still for $\xi \rightarrow 0$, one can use $\boldsymbol{w} \simeq \boldsymbol{y} \simeq(1-\theta) \boldsymbol{x}+\theta \boldsymbol{z}^{\prime}$ and $\overline{\boldsymbol{y}} \simeq \overline{\boldsymbol{z}}^{\prime}$. 

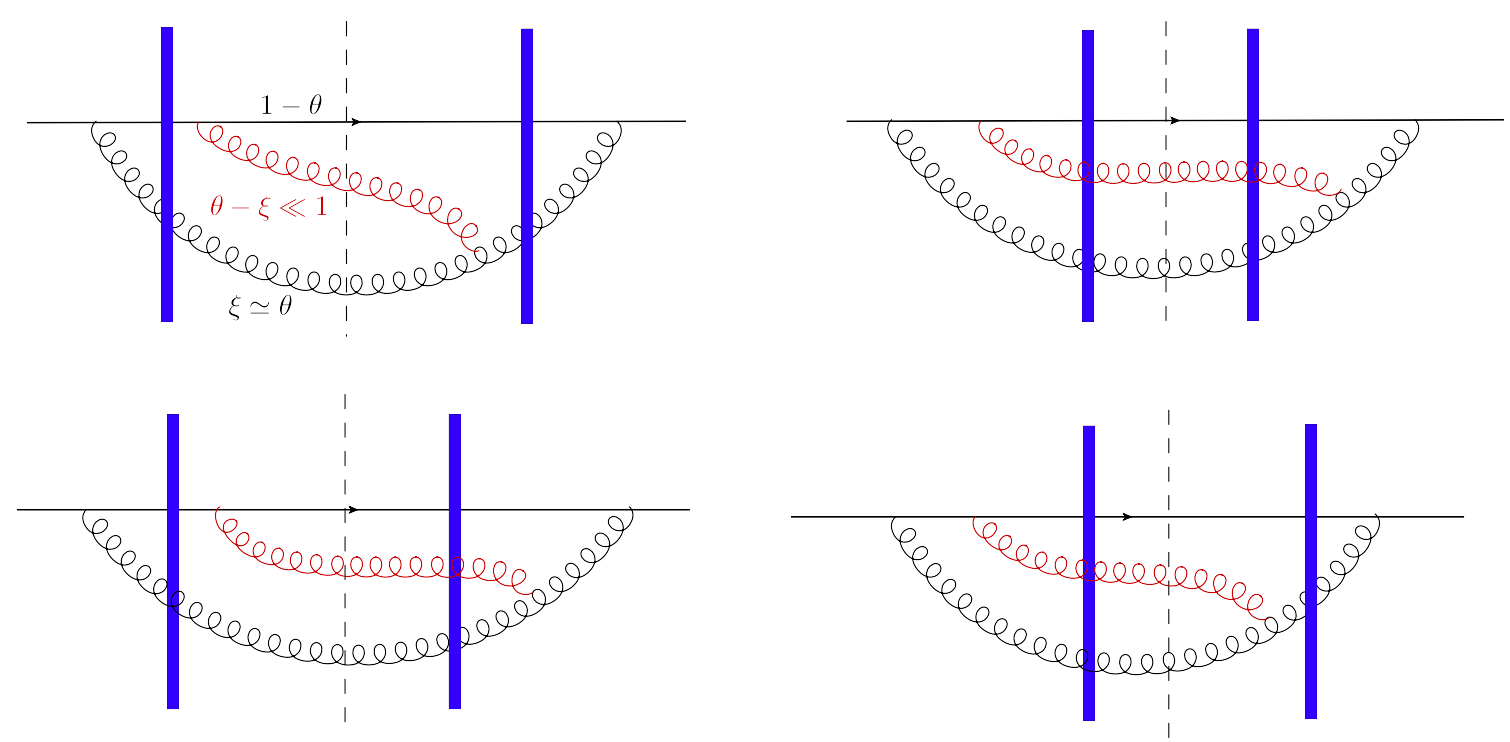

Figure 18. The four diagrams contributing to the cross-section (3.52) in the eikonal limit $\theta-\xi \rightarrow 0$ (the soft gluon is shown in red).
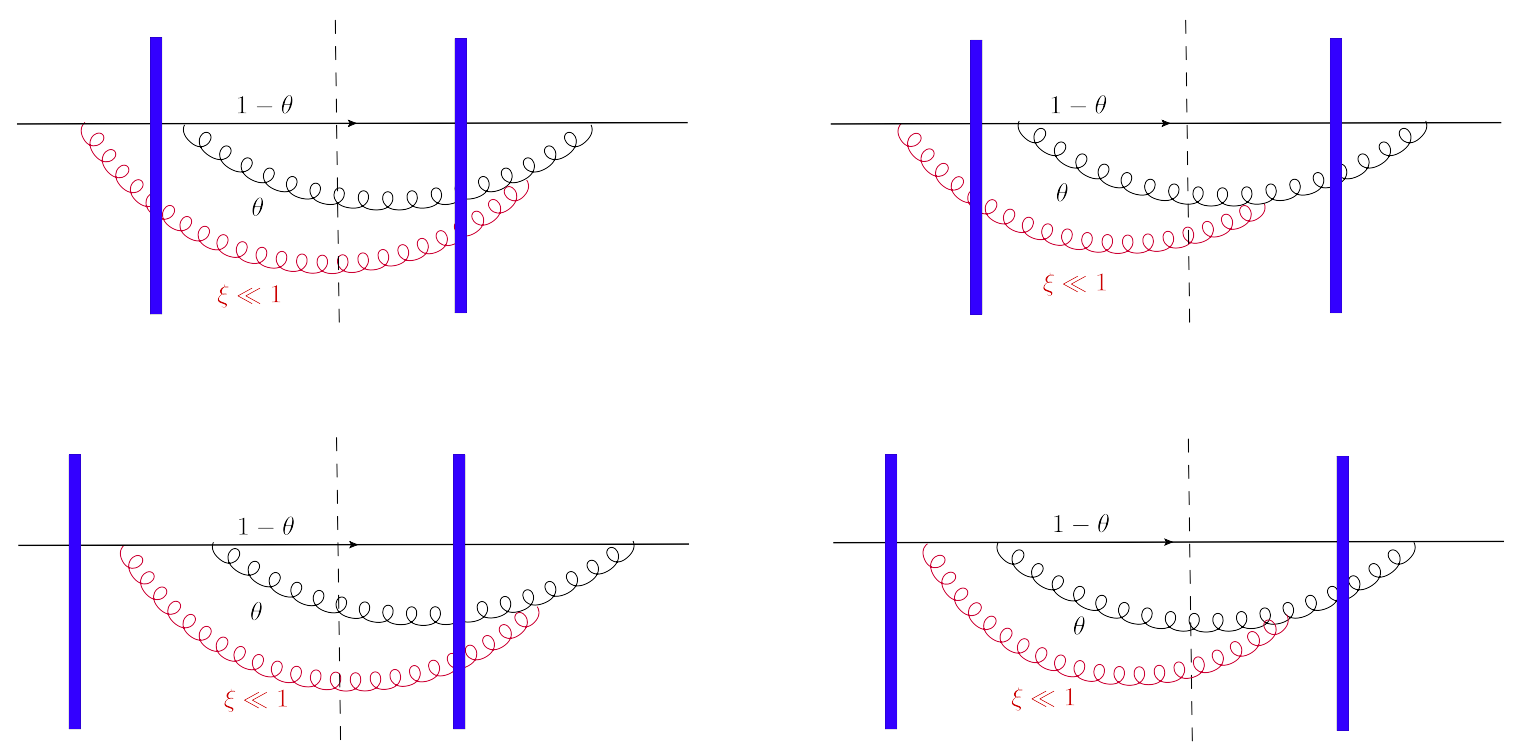

Figure 19. The four diagrams contributing to the cross-section (3.52) in the eikonal limit $\xi \rightarrow 0$ (the soft gluon is shown in red). 
To show explicit results, we shall use the momenta assignments in figure 11, which in particular imply $k_{2}^{+}=\xi q^{+}$and $k_{3}^{+}=(\theta-\xi) q^{+}$. For the case $\theta-\xi \rightarrow 0$, one easily finds

$$
\begin{aligned}
\left.\frac{d \sigma_{(3)}^{q A \rightarrow q g g+X}}{d k_{1}^{+} d^{2} \boldsymbol{k}_{1} d k_{2}^{+} d^{2} \boldsymbol{k}_{2} d k_{3}^{+} d^{2} \boldsymbol{k}_{3}}\right|_{\theta-\xi \rightarrow 0}=-\frac{4 \alpha_{s}^{2} C_{F} N_{c}}{(2 \pi)^{10}} \frac{1+(1-\theta)^{2}}{(\theta-\xi) \theta\left(q^{+}\right)^{2}} \delta\left(q^{+}-k_{1}^{+}-k_{2}^{+}\right) \\
\times \int_{\overline{\boldsymbol{x}}, \overline{\boldsymbol{z}}, \overline{\boldsymbol{z}}^{\prime}, \boldsymbol{x}, \boldsymbol{z}, \boldsymbol{z}^{\prime}} e^{-i \boldsymbol{k}_{1} \cdot(\boldsymbol{x}-\overline{\boldsymbol{x}})-i \boldsymbol{k}_{2} \cdot(\boldsymbol{z}-\overline{\boldsymbol{z}})-i \boldsymbol{k}_{3} \cdot\left(\boldsymbol{z}^{\prime}-\overline{\boldsymbol{z}}^{\prime}\right)} \frac{(\boldsymbol{X} \cdot \overline{\boldsymbol{X}})\left(\boldsymbol{X}^{\prime} \cdot \overline{\boldsymbol{Z}}\right)}{\boldsymbol{X}^{2} \overline{\boldsymbol{X}}^{2}\left(\boldsymbol{X}^{\prime}\right)^{2} \overline{\boldsymbol{Z}}^{2}} \\
\times\left[\mathcal{Q}\left(\boldsymbol{x}, \boldsymbol{z}^{\prime}, \overline{\boldsymbol{z}}^{\prime}, \overline{\boldsymbol{x}}\right) \mathcal{Q}\left(\boldsymbol{z}^{\prime}, \boldsymbol{z}, \overline{\boldsymbol{z}}, \overline{\boldsymbol{z}}^{\prime}\right)-\mathcal{Q}\left(\boldsymbol{x}, \boldsymbol{z}, \overline{\boldsymbol{z}}, \overline{\boldsymbol{z}}^{\prime}\right) \mathcal{S}\left(\overline{\boldsymbol{z}}^{\prime}, \overline{\boldsymbol{x}}\right)\right. \\
\left.\quad-\mathcal{Q}\left(\boldsymbol{x}, \boldsymbol{z}^{\prime}, \overline{\boldsymbol{z}}, \overline{\boldsymbol{x}}\right) \mathcal{S}\left(\boldsymbol{z}^{\prime}, \boldsymbol{z}\right)+\mathcal{S}(\boldsymbol{x}, \boldsymbol{z}) \mathcal{S}(\overline{\boldsymbol{z}}, \overline{\boldsymbol{x}})\right] \mathcal{S}(\boldsymbol{z}, \overline{\boldsymbol{z}}),
\end{aligned}
$$

where $\boldsymbol{X}^{\prime}=\boldsymbol{x}-\boldsymbol{z}^{\prime}, \overline{\boldsymbol{Z}}=\overline{\boldsymbol{z}}-\overline{\boldsymbol{z}}^{\prime}$, and we have also used $\boldsymbol{y}=\boldsymbol{x}$ and $\overline{\boldsymbol{y}}=\overline{\boldsymbol{z}}$ to replace $\boldsymbol{Y} \rightarrow \boldsymbol{X}=\boldsymbol{x}-\boldsymbol{z}$ and $\overline{\boldsymbol{R}} \rightarrow \overline{\boldsymbol{X}}=\overline{\boldsymbol{x}}-\overline{\boldsymbol{z}}$. These alternative notations facilitate the comparison with eq. (5.6), which refers to the direct contribution of the amplitude in figure 6 . Both eq. (5.6) and eq. (5.14) express the effect of producing one soft gluon when acting with the "production" version of the JIMWLK Hamiltonian on the quadrupole $\mathcal{Q}(\boldsymbol{x}, \boldsymbol{z}, \overline{\boldsymbol{z}}, \overline{\boldsymbol{x}})$ in the first term in the r.h.s. of eq. (2.17) for the LO dijet cross-section. ${ }^{12}$ In eq. (5.6), the soft gluon is emitted and reabsorbed by the same quark, with transverse coordinates $\boldsymbol{x}$ and $\overline{\boldsymbol{x}}$ in the DA and the CCA, respectively. Eq. (5.14) is the interference term where the soft gluon is emitted by the quark $\boldsymbol{x}$ in the DA and absorbed by the "other quark", at $\bar{z}$, in the CCA. (This "other" quark is truly a component in the large- $N_{c}$ decomposition of the harder gluon with energy fraction $\theta$.) Notice the difference in sign between eqs. (5.6) and (5.14). Except for the sign, the respective numerical factors are identical at large $N_{c}$, as they should.

Besides eqs. (5.6) and (5.14), there are two more contributions to the JIMWLK evolution of the quadrupole (here, in the sense of soft gluon production) [83]. One of them was already shown eq. (5.9), although with somewhat different notations for the transverse coordinates. ${ }^{13}$ The other one is the second interference term, in which one exchanges the topologies between the DA and the CCA; this can be easily inferred from (5.14) via hermitian conjugation. When the soft gluon is not measured, the sum of these four contributions reproduces the "real" terms in the B-JIMWLK evolution of the quadrupole [71-73].

\footnotetext{
${ }^{12}$ Notice the change of notation $\boldsymbol{y} \rightarrow \boldsymbol{z}$ when going from eq. (2.10) to eq. (5.6) or (5.14).

${ }^{13}$ The difference in notations between eq. (5.9) on one hand and eqs. (5.6) and (5.14) on the other hand comes from the fact that, in section 5.2 , we preferred to work in the other soft limit, that is, $\xi \rightarrow 0$.
} 
Consider now the other eikonal limit, namely $\xi \rightarrow 0$. Using the second line in eq. (5.13) together with the relevant terms from eq. (3.52), one finds

$$
\begin{aligned}
& \left.\frac{d \sigma_{(3)}^{q A \rightarrow q g g+X}}{d k_{1}^{+} d^{2} \boldsymbol{k}_{1} d k_{2}^{+} d^{2} \boldsymbol{k}_{2} d k_{3}^{+} d^{2} \boldsymbol{k}_{3}}\right|_{\xi \rightarrow 0}=\frac{4 \alpha_{s}^{2} C_{F} N_{c}}{(2 \pi)^{10}} \frac{1+(1-\theta)^{2}}{\xi \theta\left(q^{+}\right)^{2}} \delta\left(q^{+}-k_{1}^{+}-k_{3}^{+}\right) \\
& \times \int_{\overline{\boldsymbol{x}}, \overline{\boldsymbol{z}}, \overline{\boldsymbol{y}} \boldsymbol{x}, \boldsymbol{z}, \boldsymbol{z}^{\prime}} e^{-i \boldsymbol{k}_{1} \cdot(\boldsymbol{x}-\overline{\boldsymbol{x}})-i \boldsymbol{k}_{2} \cdot(\boldsymbol{z}-\overline{\boldsymbol{z}})-i \boldsymbol{k}_{3} \cdot\left(\boldsymbol{z}^{\prime}-\overline{\boldsymbol{y}}\right)} \frac{\left(\boldsymbol{X}^{\prime} \cdot \overline{\boldsymbol{R}}\right)(\boldsymbol{Y} \cdot \overline{\boldsymbol{Z}})}{\left(\boldsymbol{X}^{\prime}\right)^{2} \overline{\boldsymbol{R}}^{2} \boldsymbol{Y}^{2} \overline{\boldsymbol{Z}}^{2}} \\
& \times[\mathcal{S}(\boldsymbol{z}, \overline{\boldsymbol{z}}) \mathcal{Q}(\boldsymbol{y}, \boldsymbol{z}, \overline{\boldsymbol{z}}, \overline{\boldsymbol{y}})-\mathcal{S}(\boldsymbol{y}, \boldsymbol{z}) \mathcal{S}(\boldsymbol{z}, \overline{\boldsymbol{y}}) \\
& \quad-\mathcal{S}(\boldsymbol{y}, \overline{\boldsymbol{z}}) \mathcal{S}(\overline{\boldsymbol{z}}, \overline{\boldsymbol{y}})+\mathcal{S}(\boldsymbol{y}, \overline{\boldsymbol{y}})] \mathcal{S}(\overline{\boldsymbol{y}}, \overline{\boldsymbol{x}})
\end{aligned}
$$

where $\boldsymbol{Y}=\boldsymbol{y}-\boldsymbol{z}, \boldsymbol{X}^{\prime}=\boldsymbol{x}-\boldsymbol{z}^{\prime}, \overline{\boldsymbol{Z}}=\overline{\boldsymbol{z}}-\overline{\boldsymbol{z}}^{\prime}$, and $\overline{\boldsymbol{R}}=\overline{\boldsymbol{x}}-\overline{\boldsymbol{y}}$ and we have replaced $\boldsymbol{w} \rightarrow \boldsymbol{y}$ and $\overline{\boldsymbol{z}}^{\prime} \rightarrow \overline{\boldsymbol{y}}$, as appropriate in this limit. The $S$-matrices within the square brackets have the right structure to describe the production of a soft gluon from the dipole $\mathcal{S}(\boldsymbol{y}, \overline{\boldsymbol{y}})$ (see eq. (A.2) in ref. [83]).

If the soft gluon is not measured in the final state, one must integrate eq. (5.15) over $k_{2}^{+}=\xi q^{+}$and $\boldsymbol{k}_{2} ;$ this gives (with $2 \alpha_{s} C_{F} \simeq \pi \bar{\alpha}_{s}$ )

$$
\begin{aligned}
\frac{d \sigma_{\mathrm{NLO}, 3}^{q A \rightarrow q g+X}}{d k_{1}^{+} d^{2} \boldsymbol{k}_{1} d k_{3}^{+} d^{2} \boldsymbol{k}_{3}} \simeq & -\frac{\bar{\alpha}_{s}}{(2 \pi)^{5}} \frac{1+(1-\theta)^{2}}{2 \theta q^{+}} \delta\left(q^{+}-k_{1}^{+}-k_{3}^{+}\right) \\
& \times \int_{\overline{\boldsymbol{x}}, \overline{\boldsymbol{y}}, \boldsymbol{x}, \boldsymbol{z}^{\prime}} e^{-i \boldsymbol{k}_{1} \cdot(\boldsymbol{x}-\overline{\boldsymbol{x}})-i \boldsymbol{k}_{3} \cdot\left(\boldsymbol{z}^{\prime}-\overline{\boldsymbol{y}}\right)} \frac{\left(\boldsymbol{x}-\boldsymbol{z}^{\prime}\right) \cdot(\overline{\boldsymbol{x}}-\overline{\boldsymbol{y}})}{\left(\boldsymbol{x}-\boldsymbol{z}^{\prime}\right)^{2}(\overline{\boldsymbol{x}}-\overline{\boldsymbol{y}})^{2}} \mathcal{S}(\overline{\boldsymbol{y}}, \overline{\boldsymbol{x}}) \\
& \times \frac{\bar{\alpha}_{s}}{2 \pi} \int_{0}^{1} \frac{d \xi}{\xi} \int_{\boldsymbol{z}} \frac{2(\boldsymbol{y}-\boldsymbol{z}) \cdot(\overline{\boldsymbol{y}}-\boldsymbol{z})}{(\boldsymbol{y}-\boldsymbol{z})^{2}(\overline{\boldsymbol{y}}-\boldsymbol{z})^{2}}[\mathcal{S}(\boldsymbol{y}, \overline{\boldsymbol{y}})-\mathcal{S}(\boldsymbol{y}, \boldsymbol{z}) \mathcal{S}(\boldsymbol{z}, \overline{\boldsymbol{y}})],
\end{aligned}
$$

where the change of global sign occurred because $\overline{\boldsymbol{Z}}=-(\overline{\boldsymbol{y}}-\boldsymbol{z})$. This is indeed the right sign to describe the BK evolution of the dipole $\mathcal{S}(\boldsymbol{w}, \overline{\boldsymbol{y}})$ from the product $\mathcal{S}(\boldsymbol{w}, \overline{\boldsymbol{y}}) \mathcal{S}(\overline{\boldsymbol{y}}, \overline{\boldsymbol{x}})$ which appears with a negative sign in the third term in the r.h.s. of eq. (2.17). (Recall that $\boldsymbol{w} \simeq \boldsymbol{y}$ in the soft limit at hand.)

To summarise, the discussion of the eikonal limits of our results for the qgg trijet cross-section has allowed us to reconstruct the "real" terms in the B-JIMWLK evolution of the LO cross-section (2.17) for the $q g$ dijet. Specifically, we have identified the "real" part of the evolution equations for the first term (the product $\mathcal{Q}(\boldsymbol{x}, \boldsymbol{y}, \overline{\boldsymbol{y}}, \overline{\boldsymbol{x}}) \mathcal{S}(\boldsymbol{y}, \overline{\boldsymbol{y}})$ between a quadrupole and a dipole) and for the last term (the dipole $\mathcal{S}(\boldsymbol{w}, \overline{\boldsymbol{w}})$ ) in eq. (2.17), and also for the dipoles $\mathcal{S}(\boldsymbol{y}, \overline{\boldsymbol{w}})$ and $\mathcal{S}(\boldsymbol{w}, \overline{\boldsymbol{y}})$ which appears in the two intermediate terms there. On the other hand, we have not yet encountered the evolution of the two other dipoles which enter these intermediate terms, namely $\mathcal{S}(\boldsymbol{x}, \boldsymbol{y})$ and $\mathcal{S}(\overline{\boldsymbol{y}}, \overline{\boldsymbol{x}})$. There is a good reason for that: these two other dipoles are built with quark legs which "live" on the same side of the cut, that is, they both "live" in the DA for $\mathcal{S}(\boldsymbol{x}, \boldsymbol{y})$, and respectively in the CCA for $\mathcal{S}(\overline{\boldsymbol{y}}, \overline{\boldsymbol{x}})$. Accordingly, the BK evolution of these dipoles involves graphs like those shown in figure 20, which represent virtual corrections to the $q g$ dijet cross-section. The contributions of such graphs will be considered in a companion paper devoted to virtual corrections. 

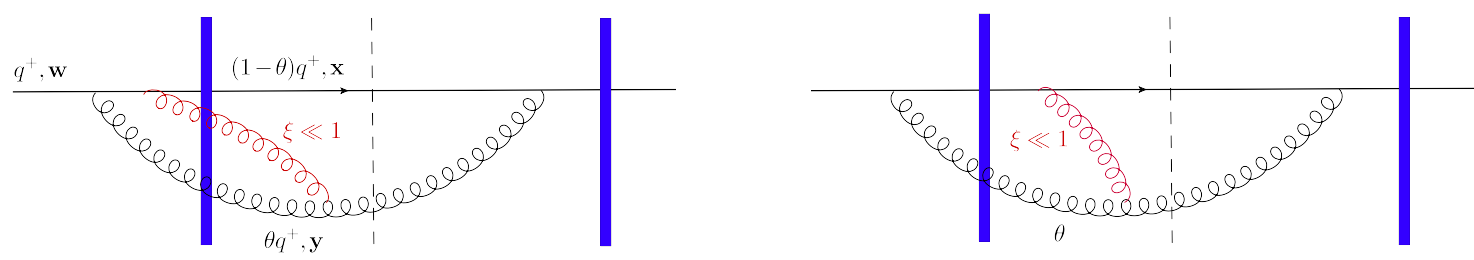

Figure 20. Graphs representing virtual corrections to the $q g$ dijet cross-section. Both shown graphs count for the BK evolution of the dipole $\mathcal{S}(\boldsymbol{x}, \boldsymbol{y})$ which enters the second term in the r.h.s. of eq. (2.17).

\section{Collinear divergences: recovering the DGLAP evolution}

Besides the soft divergences that we have just discussed, the NLO corrections to the dijet cross-section are expected to also contain collinear divergences. In momentum space, they correspond to the limit where the daughter partons emerging from a splitting have a very small relative transverse momentum (i.e. they make a very small angle $\theta \rightarrow 0$ ). In the transverse coordinate space, this is the limit where the transverse separation between the two daughter partons becomes arbitrarily large.

Before turning to explicit calculations, let us first recall some general features about the origin and the treatment of the collinear divergences in the framework of the hybrid factorisation (see e.g. [21]):

(i) collinear divergences are associated with (unmeasured) partons which are emitted long before the hard process, or longtime after it. By the "hard process", we mean the ensemble of the other interactions, that is, the scattering off the nuclear target (the shockwave) and the emission of additional, relatively hard (non-collinear), partons.

(ii) The "collinear" parton may scatter with the shockwave, but its scattering does not matter for the inclusive dijet cross-section at NLO.

(iii) If the collinear emission occurs prior to the hard process - in the present case, that should be a gluon emission by the incoming quark -, then the associated divergence can be reabsorbed into the DGLAP evolution of the parton distributions in the proton projectile (here, the quark distribution $q_{f}\left(x_{q}, \mu^{2}\right)$, as visible in eqs. (2.16) and (4.6)).

(iv) If the collinear emission originates from one of the final partons - in this case, that could be either a gluon emission from the final quark, or the splitting of the final gluon into two gluons, or into a quark-antiquark pair -, then its treatment depends upon our definition of the measurement process. If we measure two hadrons in the final state, then the collinear divergences should be absorbed into the DGLAP evolution of the fragmentation functions for the quark, or the gluon, emerging from the hard process. If instead we measure two jets, whose definition involves an opening angle $R$, then any emission at small angles $\theta<R$ must be viewed as a part of the final-state jets, whereas emissions at larger angles $\theta>R$ are interpreted as NLO corrections to the hard process. In this argument, the jet angle $R$ effectively acts as a collinear 

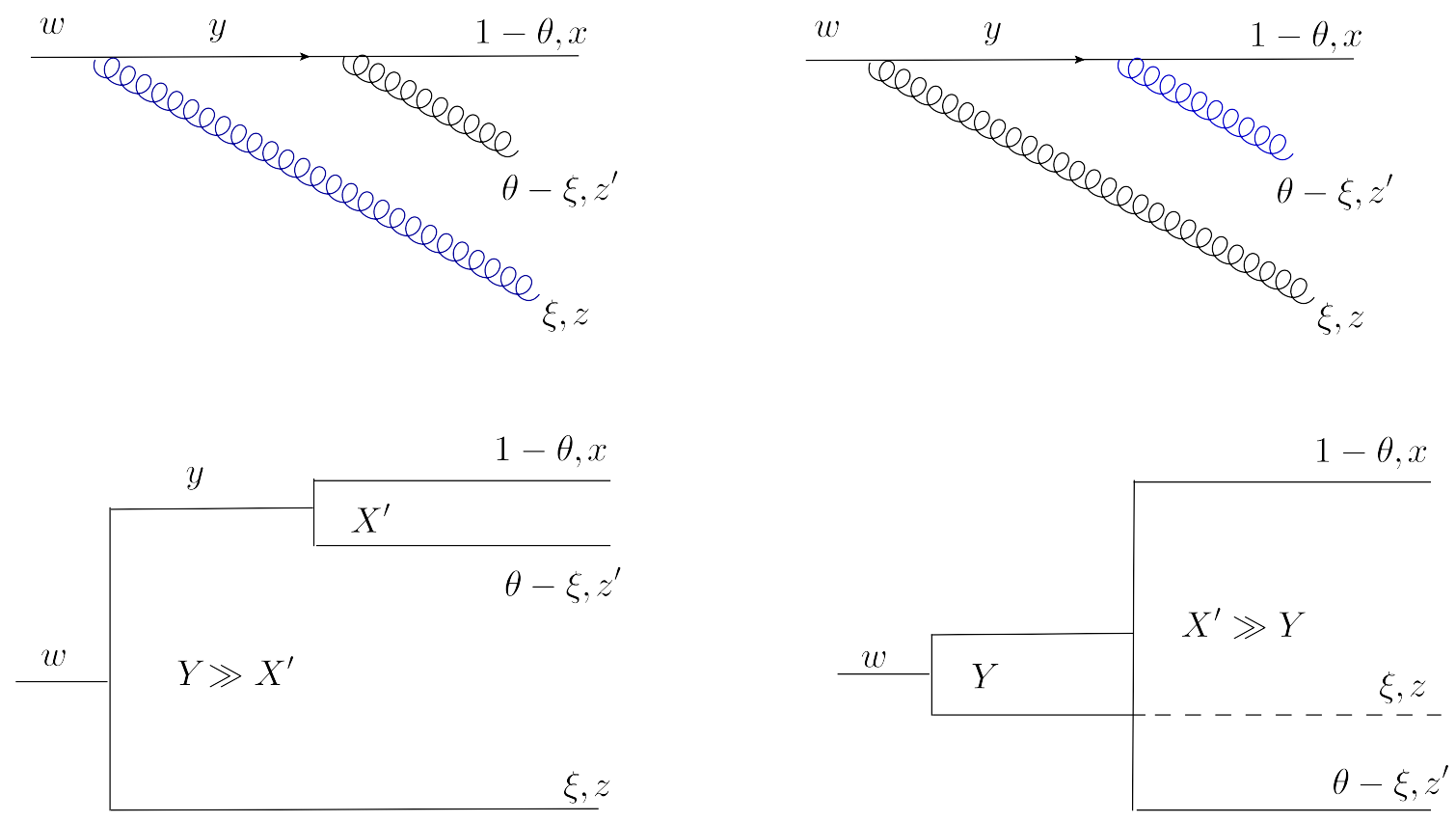

Figure 21. Graphical illustration of the collinear limits corresponding to the two gluon emissions by the incoming quark. The Feynman graphs in the first line show the topology of the emission: the gluon emerging from a collinear splitting is represented in blue. The schematic diagrams in the second line illustrate the geometry of the splittings in the transvserse coordinate space.

cutoff for the NLO corrections. In this remaining part of this section, we shall assume a hadronic description for the final state, for definiteness.

(v) The collinear divergences can be unambiguously separated from the infrared (or "soft") divergences associated with soft gluons. A given graph can develop both soft and collinear divergences, but the overlapping divergences cancel - as a consequence of probability conservation - when adding together real and virtual corrections. This makes it possible to disentangle soft from collinear divergences in practice and to ascribe them to the B-JIMWLK evolution of the hard process and to the DGLAP evolutions of the initial and final states, respectively.

\subsection{DGLAP evolution for the initial quark}

Our first example refers the NLO contribution denoted with a subscript (1) in eq. (4.16). We recall that this contribution is obtained from the trijet cross-section (3.39) by integrating out a gluon with momentum $k_{3}$, which can be any of the two gluons in the final state, cf. figure 9 .

As already mentioned, the collinear regime corresponds to the situation where the transverse separation between the two daughter partons can be arbitrarily large. For the diagram in figure 9, there are two such limits, one for each of the two gluon emissions, as illustrated in figure 21. In the first case, depicted in the l.h.s. of figure 21, the first gluon emission is the collinear one; one then has $|\boldsymbol{Y}| \gg\left|\boldsymbol{X}^{\prime}\right|$, where we recall that $\boldsymbol{X}^{\prime}=\boldsymbol{x}-\boldsymbol{z}^{\prime}$ 

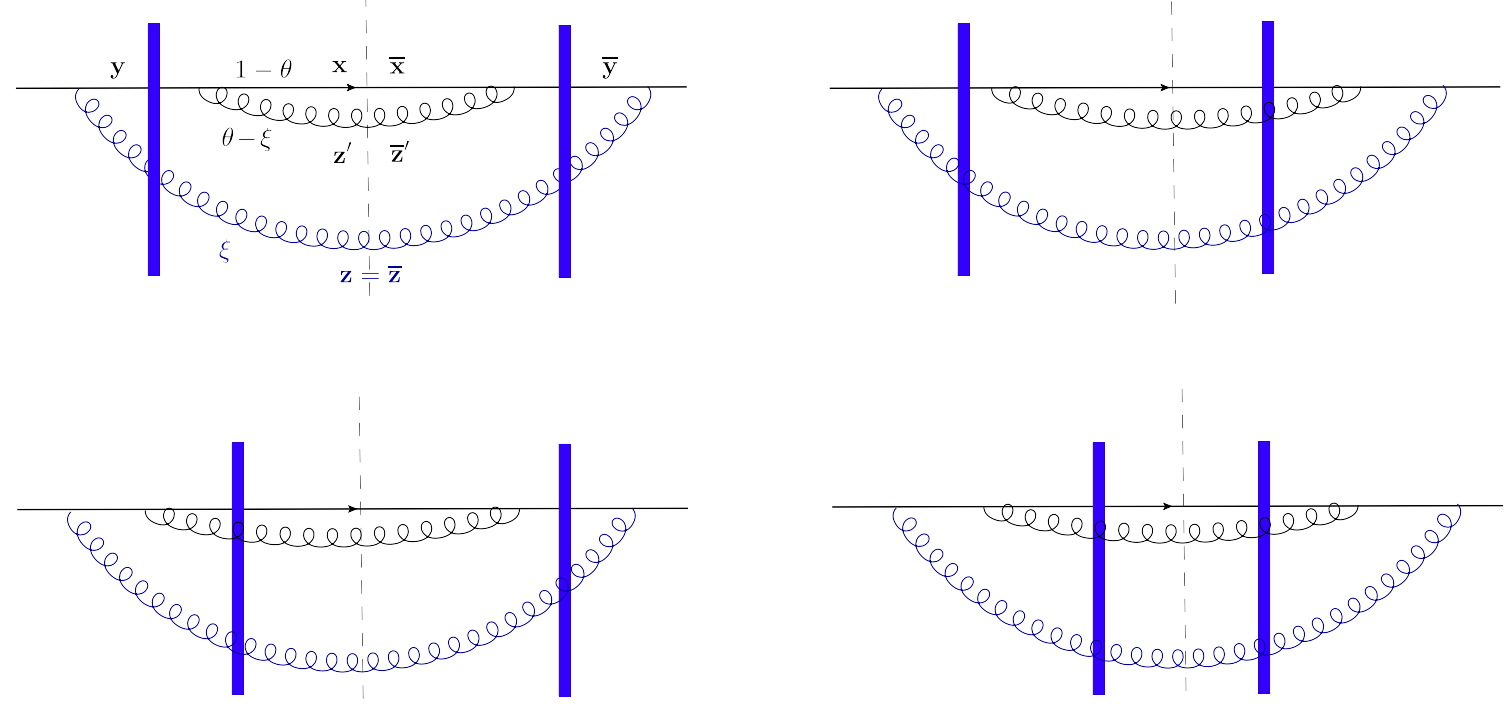

Figure 22. The four graphs contributing to the limit where the first gluon emission (depicted in blue) is collinear. These graphs are free of initial-state interactions.

and $\boldsymbol{Y}=\boldsymbol{y}-\boldsymbol{z}$. This covers the case of the DGLAP evolution of the incoming quark distribution, to be discussed in this section. In the other case (the r.h.s. of figure 21), the opposite inequality holds: $\left|\boldsymbol{X}^{\prime}\right| \gg|\boldsymbol{Y}|$. This is the case of the final state DGLAP evolution, to be discussed in the next subsection.

Consider the case depicted in the 1.h.s. of figure 21: the first gluon emission, with longitudinal fraction $\xi$, is not measured $(\overline{\boldsymbol{z}}=\boldsymbol{z})$ and is in the collinear regime. Using $\boldsymbol{Y}^{2} \gg\left(\boldsymbol{X}^{\prime}\right)^{2}$ and similarly $\overline{\boldsymbol{Y}}^{2} \gg\left(\overline{\boldsymbol{X}}^{\prime}\right)^{2}$, one finds that the kernel (3.40) simplifies to (recall eq. (3.41))

$$
\mathcal{K}_{1}^{m n p q} \simeq \frac{32 \delta^{m p} \delta^{n q}}{(1-\xi) \boldsymbol{Y}^{2} \overline{\boldsymbol{Y}}^{2}} P_{q \rightarrow g}(\xi) P_{q \rightarrow g}\left(\xi^{\prime}\right)
$$

where $\xi^{\prime} \equiv(\theta-\xi) /(1-\xi)$ denotes the splitting fraction for the second gluon emission and

$$
P_{q \rightarrow g}(z) \equiv \frac{1+(1-z)^{2}}{2 z},
$$

is the DGLAP splitting function for the process $q \rightarrow q g$. The approximation in eq. (6.1) holds so long as

$$
\xi(1-\xi)^{2} \boldsymbol{Y}^{2} \gg(1-\theta)(\theta-\xi)\left(\boldsymbol{X}^{\prime}\right)^{2},
$$

together with a similar condition involving $\overline{\boldsymbol{Y}}$ and $\overline{\boldsymbol{X}}^{\prime}$. In writing eq. (6.1), we have ignored the instantaneous pieces of the effective vertices which enter the numerator of $\mathcal{K}_{1}$ (that is, we have used eq. (3.41)). On physical grounds, it is quite obvious that these pieces cannot yield collinear singularities: they correspond to effective graphs in which the two emissions occur simultaneously. We shall shortly give a more mathematical argument in that sense. 
Eq. (6.1) shows that, in the collinear limit, ${ }^{14}$ the two gluon emissions factorise from each other at the level of the kernel $\mathcal{K}_{1}$. Let us now check that a similar factorisation also holds at the level of the colour structure, i.e. for the $S$-matrices.

Since all the four terms within the squared brackets in eq. (3.39) are now multiplied by a same kernel, it follows that only four topologies (in terms of shockwave insertions) survive in this limit. These are the topologies shown in figure 22, which do not involve initial-state interactions, as expected: the "collinear" gluon is emitted before the hard process, both in the DA and in the CCA. This gluon crosses the shockwave in all these graphs, but the Wilson lines describing its scattering mutually cancel by unitarity: $V(\boldsymbol{z}) V^{\dagger}(\overline{\boldsymbol{z}})=1$ when $\overline{\boldsymbol{z}}=\boldsymbol{z}$. Because of this cancellation, the colour structure of the four graphs in figure 22 is precisely the same as for the LO $q g$ final state in eq. (2.10).

Using eqs. (3.39) and (6.1), one deduces the following approximation for this particular dijet cross-section in the collinear limit for the unmeasured gluon:

$$
\begin{aligned}
& \frac{d \sigma_{(1) \mathrm{rNLO}, 1}^{q A \rightarrow q g+X}}{d p^{+} d^{2} \boldsymbol{p} d k^{+} d^{2} \boldsymbol{k}} \simeq \frac{4 \alpha_{s} C_{F}}{(2 \pi)^{6}\left(q^{+}\right)^{2}(1-\xi)} P_{q \rightarrow g}\left(\frac{x_{2}}{x_{1}+x_{2}}\right) \\
& \quad \times \int_{\overline{\boldsymbol{x}}, \overline{\boldsymbol{z}}^{\prime}, \boldsymbol{x}, \boldsymbol{z}^{\prime}} e^{-i \boldsymbol{p} \cdot(\boldsymbol{x}-\overline{\boldsymbol{x}})-i \boldsymbol{k} \cdot\left(\boldsymbol{z}^{\prime}-\overline{\boldsymbol{z}}^{\prime}\right)} \frac{\left(\boldsymbol{x}-\boldsymbol{z}^{\prime}\right) \cdot\left(\overline{\boldsymbol{x}}-\overline{\boldsymbol{z}}^{\prime}\right)}{\left(\boldsymbol{x}-\boldsymbol{z}^{\prime}\right)^{2}\left(\overline{\boldsymbol{x}}-\overline{\boldsymbol{z}}^{\prime}\right)^{2}} \\
& \times\left[\mathcal{Q}\left(\boldsymbol{x}, \boldsymbol{z}^{\prime}, \overline{\boldsymbol{z}}^{\prime}, \overline{\boldsymbol{x}}\right) \mathcal{S}\left(\boldsymbol{z}^{\prime}, \overline{\boldsymbol{z}}^{\prime}\right)-\mathcal{S}\left(\boldsymbol{x}, \boldsymbol{z}^{\prime}\right) \mathcal{S}\left(\boldsymbol{z}^{\prime}, \overline{\boldsymbol{y}}\right)-\mathcal{S}\left(\boldsymbol{y}, \overline{\boldsymbol{z}}^{\prime}\right) \mathcal{S}\left(\overline{\boldsymbol{z}}^{\prime}, \overline{\boldsymbol{x}}\right)+\mathcal{S}(\boldsymbol{y}, \overline{\boldsymbol{y}})\right] \\
& \quad \times \frac{4 \alpha_{s} C_{F}}{(2 \pi)^{2}} P_{q \rightarrow g}(\xi) \int_{\boldsymbol{z}} \frac{(\boldsymbol{y}-\boldsymbol{z}) \cdot(\overline{\boldsymbol{y}}-\boldsymbol{z})}{(\boldsymbol{y}-\boldsymbol{z})^{2}(\overline{\boldsymbol{y}}-\boldsymbol{z})^{2}} .
\end{aligned}
$$

For more clarity, we have used the same notations for the momenta of the two produced partons as in eq. $(2.10):\left(p^{+}, \boldsymbol{p}\right)$ and $\left(k^{+}, \boldsymbol{k}\right)$ refer to the produced quark and gluon, respectively. Furthermore, $x_{1}=p^{+} / Q^{+}$and $x_{2}=k^{+} / Q^{+}$, with $Q^{+}$the longitudinal momentum of the incoming proton. The original longitudinal fractions $\theta$ and $\xi$ should now be evaluated as

$$
\theta=1-\frac{x_{1}}{x_{q}}, \quad \xi=1-\frac{x_{1}+x_{2}}{x_{q}} \Longrightarrow \xi^{\prime} \equiv \frac{\theta-\xi}{1-\xi}=\frac{x_{2}}{x_{1}+x_{2}},
$$

where we recall that $x_{q} \equiv q^{+} / Q^{+}$denotes the longitudinal momentum fraction of the original quark. The transverse coordinate $\boldsymbol{y}$ of the intermediate quark is related to the coordinates $\boldsymbol{x}$ and $\boldsymbol{z}^{\prime}$ of the produced partons via eq. (3.16), which becomes

$$
\left.\boldsymbol{y}=\frac{x_{1} \boldsymbol{x}+x_{2} \boldsymbol{z}^{\prime}}{x_{1}+x_{2}} \quad \text { (and similarly for } \overline{\boldsymbol{y}}\right) \text {. }
$$

Eq. (6.4) exhibits the expected factorisation of the (unmeasured) collinear emission from the LO dijet cross-section (2.10) - here initiated by a quark with original longitudinal momentum $q^{+}(1-\xi)$. The integral over $\boldsymbol{z}$ in the last line, which physically represents the integral over the transverse phase space for the collinear emission, is logarithmically divergent at large $|\boldsymbol{z}|$. To exhibit this singularity, let us introduce a low-momentum cutoff

\footnotetext{
${ }^{14}$ In general, i.e. beyond the collinear limit, this factorisation would be spoilt by the dependence of the energy denominator in eq. (3.40) upon the longitudinal fractions $\theta$ and $\xi$.
} 
$\Lambda$ on the transverse momentum of the unmeasured gluon, meaning an upper cutoff $\sim 1 / \Lambda$ on the transverse separations $|\boldsymbol{y}-\boldsymbol{z}|$ and $|\overline{\boldsymbol{y}}-\boldsymbol{z}|$. With this regularisation, the integral over $\boldsymbol{z}$ in eq. (6.4) can be estimated as (below, $r \equiv|\boldsymbol{y}-\overline{\boldsymbol{y}}|$ )

$$
\int d^{2} \boldsymbol{z} \frac{(\boldsymbol{y}-\boldsymbol{z}) \cdot(\overline{\boldsymbol{y}}-\boldsymbol{z})}{(\boldsymbol{y}-\boldsymbol{z})^{2}(\overline{\boldsymbol{y}}-\boldsymbol{z})^{2}} \rightarrow \int \frac{d^{2} \boldsymbol{q}}{\boldsymbol{q}^{2}} e^{-i \boldsymbol{q} \cdot(\boldsymbol{y}-\overline{\boldsymbol{y}})} \Theta(q-\Lambda)=\pi \ln \frac{1}{r^{2} \Lambda^{2}}=\pi \ln \frac{1}{r^{2} \mu^{2}}+\pi \ln \frac{\mu^{2}}{\Lambda^{2}} .
$$

In the last equality, $\mu$ is a generic scale obeying $\Lambda \ll \mu<1 / r$. In what follows, it will be used as a renormalisation scale to subtract the collinear divergence at $\Lambda \rightarrow 0$ from the genuine NLO correction. The divergence will be then absorbed into the DGLAP evolution of the quark distribution for the incoming quark.

To that aim, one should recall that the physical cross-section also includes a convolution with $q_{f}\left(x_{q}, \mu^{2}\right)$, as visible in eq. (2.16) at LO and in eq. (4.6) for a particular "real" NLO correction. For the NLO correction at hand and to the perturbative accuracy of interest, this convolution reads

$$
\int_{0}^{1} d x_{q} q_{f}^{(0)}\left(x_{q}\right) \Theta\left(x_{q}-x_{1}-x_{2}\right)(\cdots)=\int_{0}^{1} \frac{d \xi}{1-\xi} \frac{x_{1}+x_{2}}{1-\xi} q_{f}^{(0)}\left(\frac{x_{1}+x_{2}}{1-\xi}\right) \Theta\left(1-\xi-x_{1}-x_{2}\right)(\cdots),
$$

where $q_{f}^{(0)}\left(x_{q}\right)$ is the bare (parton model) quark distribution, which is independent of the scale $\mu^{2}$, and the dots within the integrand stay for the partonic cross-section in eq. (6.4). In the integral in the r.h.s. we changed variable $x_{q} \rightarrow \xi$ according to (6.5); the step function comes from the condition $x_{q} \leq 1$. When using this convolution together with eq. (6.4), one should also notice that $q^{+}(1-\xi)=p^{+}+k^{+}=\left(x_{1}+x_{2}\right) Q^{+}$is the total longitudinal momentum of the produced dijet and thus it is independent of $\xi$. Hence, the whole $\xi$ dependence of the final result is encoded in the following integral

$$
x \Delta q_{f}\left(x, \mu^{2}\right) \equiv \frac{\alpha_{s} C_{F}}{\pi} \int_{0}^{1-x} d \xi \frac{x}{1-\xi} q_{f}^{(0)}\left(\frac{x}{1-\xi}\right) P_{q \rightarrow g}(\xi) \ln \frac{\mu^{2}}{\Lambda^{2}},
$$

where it is understood that $x \equiv x_{1}+x_{2}$. As suggested by its notation, the r.h.s. in (6.9) is precisely the result of one "real" step in the DGLAP evolution of the quark distribution inside the proton, where the step consists in integrating out gluon radiation with virtualities $q^{2}$ within the range $\Lambda^{2}<q^{2}<\mu^{2}$. The corresponding virtual graphs are expected to add the "plus" prescription to the splitting function $P_{q \rightarrow g}(\xi)$ and thus remove the apparent divergence of (6.9) at $\xi \rightarrow 0$. This will be checked in our subsequent paper (see also the discussion at the end of this subsection).

By inspection of eqs. (6.4)-(6.9), one can see that the original collinear divergence from the NLO correction has been transferred to the DGLAP evolution of the incoming quark distribution in the $\mathrm{LO}$ result. The finite reminder, as obtained by keeping only the second term, $\pi \ln \left(1 / r^{2} \mu^{2}\right)$, in the r.h.s. of eq. (6.7), represents a genuine NLO correction to the hard process. However, our above calculation for this correction is only correct to leading logarithmic accuracy w.r.t. the logarithm $\ln \left(p_{h}^{2} / \mu^{2}\right)$, with $p_{h}^{2} \equiv \max \left(\boldsymbol{p}^{2}, \boldsymbol{k}^{2}\right)$. (The typical value of $r \equiv|\boldsymbol{y}-\overline{\boldsymbol{y}}|$ is fixed by the Fourier transforms in (6.4) as $r^{2} \sim 1 / p_{h}^{2}$.) The $\mu$-dependence of the cross-section introduced by this and other similar corrections 
compensates the respective dependence of the quark distribution. This ensures that the result for the cross-section is independent of the arbitrary renormalisation scale $\mu$ at NLO.

In order to have a complete NLO result, which also includes the numerical coefficient under the log, one must go beyond our previous approximations in eqs. (6.1) or (6.7). That is, one should exactly compute the contributions from graphs which contain collinear divergences, like the 4 graphs figure 22, by using dimensional regularisation together with a suitable subtraction scheme, like $\overline{M S}$.

At this level, it is easy to understand why the graphs involving instantaneous propagators cannot generate collinear divergences. As clear by inspection of (6.4), such divergences occur via the integration over the Weiszäcker-Williams kernel $\boldsymbol{Y}^{m} / \boldsymbol{Y}^{2}$ which describes a bremsstrahlung process in transverse space (recall e.g. eq. (3.39)). This kernel is inherent in the graphs involving the regular part of the (quark or gluon) propagator, but it is absent from the instantaneous graphs. Said differently, the contribution from the instantaneous vertices do not involve enough powers of $\boldsymbol{Y}^{m}$ and/or $\overline{\boldsymbol{Y}}^{p}$ to generate a divergence when integrating over $\boldsymbol{z}$.

It is finally interesting to compare the graphs in figure 22 , in which the first emitted gluon is collinear, to those in figure 12, where the same gluon is soft. In figure 22 there are no initial-state interactions, whereas in figure 12 the final-state interactions are missing. This is in agreement with the physical expectations that a collinear gluon must be emitted very early, well before the hard process, unlike a soft gluon, which must be the closest emission with respect to the collision. This physical distinction should allow us to unambiguously separate between soft and collinear divergences.

Yet, by inspection of these two figures, one sees that one particular graph contributes in both cases: this is the first graph in both figure 22 and figure 12. This graph has generated the term proportional to $\mathcal{S}(\boldsymbol{y}, \overline{\boldsymbol{y}})$ in eq. (6.4), which is in fact an exact evaluation for that graph $^{15}$ - up to regularisation issues, of course. And indeed, if one takes the soft limit $\xi \rightarrow 0$ in eq. (6.4), one recovers one of the two terms proportional to $\mathcal{S}(\boldsymbol{w}, \overline{\boldsymbol{w}})$ in eq. (5.5). (The other such a term comes the fourth graph in figure 12, in which the soft gluon is never intersecting the shockwave. We recall that, in writing eq. (5.5), we have identified $\boldsymbol{y}=\boldsymbol{w}$ and $\overline{\boldsymbol{y}}=\overline{\boldsymbol{w}}$.)

This discussion makes clear that the first graph in figure 22 (or in figure 12) contributes to both the collinear (DGLAP) and the soft (B-JIMWLK) evolutions. In particular, it contains an overlapping, soft and collinear, divergence. This might seem to contradict our previous argument, that these two types of divergences can be separated from each other. In fact, there is no contradiction: such overlapping divergence are eventually cancelled after adding the virtual corrections.

We shall systematically study the virtual corrections in a subsequent paper. Here we merely show in figure 23 the graphs relevant to the above discussion: the real graph that has already appeared as the first diagram figure 22 together with the virtual graphs which remove its collinear divergences in the limit where the first emission is both collinear and

\footnotetext{
${ }^{15}$ Remember that this particular graph originates from the last term within the square brackets in (3.39), for which eq. (6.1) becomes exact.
} 

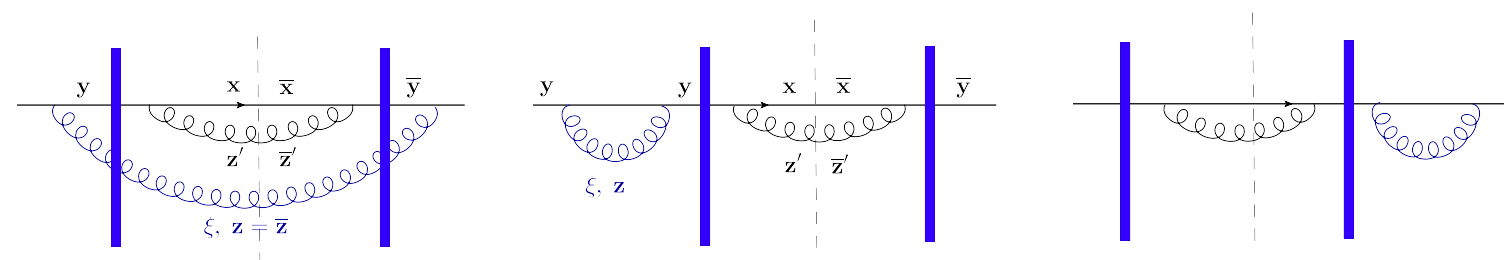

Figure 23. The set of "real" and "virtual" graphs which count for the cancellation of the overlapping, soft and collinear, divergences associated with the first gluon emission by the quark.

soft $(\xi \ll 1)$. The soft limit is indeed important for this argument: in general, the real NLO corrections and the virtual ones are weighted by quark distribution functions with different arguments: $x_{q}=\left(x_{1}+x_{2}\right) /(1-\xi)$ for the real corrections, cf. eq. (6.8), but $x_{q}=x_{1}+x_{2}$ for the virtual graphs, whose final state involves only two partons: the measured quark and gluon. However, when $\xi \ll 1$, these two weighting factors become identical with each other and then it becomes possible to observe the cancellation of the overlapping, soft and collinear, divergences.

Let us show how this works for the three graphs in figure 23. Each of the two virtual graphs gives a contribution which is simply the product between the LO dijet crosssection (2.10) and a "tadpole" describing the virtual gluon emission. The effect of adding these contributions to the real term in eq. (6.4) is to replace the emission kernel for the first emission, cf. eq. (6.7), by the dipole kernel,

$$
\mathcal{M}(\boldsymbol{y}, \overline{\boldsymbol{y}}, \boldsymbol{z}) \equiv \frac{1}{(\boldsymbol{y}-\boldsymbol{z})^{2}}+\frac{1}{(\overline{\boldsymbol{y}}-\boldsymbol{z})^{2}}-2 \frac{(\boldsymbol{y}-\boldsymbol{z}) \cdot(\overline{\boldsymbol{y}}-\boldsymbol{z})}{(\boldsymbol{y}-\boldsymbol{z})^{2}(\overline{\boldsymbol{y}}-\boldsymbol{z})^{2}}=\frac{(\boldsymbol{y}-\overline{\boldsymbol{y}})^{2}}{(\boldsymbol{y}-\boldsymbol{z})^{2}(\overline{\boldsymbol{y}}-\boldsymbol{z})^{2}},
$$

which decreases much faster at large $|\boldsymbol{z}|$ then the original kernel in eq. (6.7): as $1 / \boldsymbol{z}^{4}$ instead of $1 / \boldsymbol{z}^{2}$. So, the would-be logarithmic singularity at large $|\boldsymbol{z}|$ disappears. The dipole kernel exhibits instead short-distance (ultraviolet) poles at $\boldsymbol{z}=\boldsymbol{y}$ and $\boldsymbol{z}=\overline{\boldsymbol{y}}$, but they are ultimately inocuous, as they cancel against other virtual corrections, not shown in figure 23.

\subsection{DGLAP evolution for the final quark}

Still for the topology illustrated in figure 9, we now consider the case where the second gluon emission - the one where the gluons carries a longitudinal momentum fraction $\theta-\xi$ in figure 9 - is not measured $\left(\overline{\boldsymbol{z}}^{\prime}=\boldsymbol{z}^{\prime}\right)$ and is collinear. As already explained, this means that the transverse separation $\boldsymbol{X}^{\prime}=\boldsymbol{x}-\boldsymbol{z}^{\prime}$ between the two daughter partons emerging from the second splitting is much larger than the corresponding separation $\boldsymbol{Y}=\boldsymbol{y}-\boldsymbol{z}$ for the first emission: $\left(\boldsymbol{X}^{\prime}\right)^{2} \gg(\boldsymbol{Y})^{2}$. Under this assumption, the dominant contribution to the trijet cross-section in eq. (3.39) comes from the last term there - the one obtained after the double replacement $\left(\boldsymbol{x}, \boldsymbol{z}^{\prime} \rightarrow \boldsymbol{y} \& \overline{\boldsymbol{x}}, \overline{\boldsymbol{z}}^{\prime} \rightarrow \overline{\boldsymbol{y}}\right)$ - since the corresponding kernel is the only one not to be suppressed at large $\left(\boldsymbol{X}^{\prime}\right)^{2}$. Not surprisingly, the graphs which survive in this limit are those which are void of final state interactions, as illustrated in figure 24. We therefore expect the collinear divergence generated by these graphs to express 

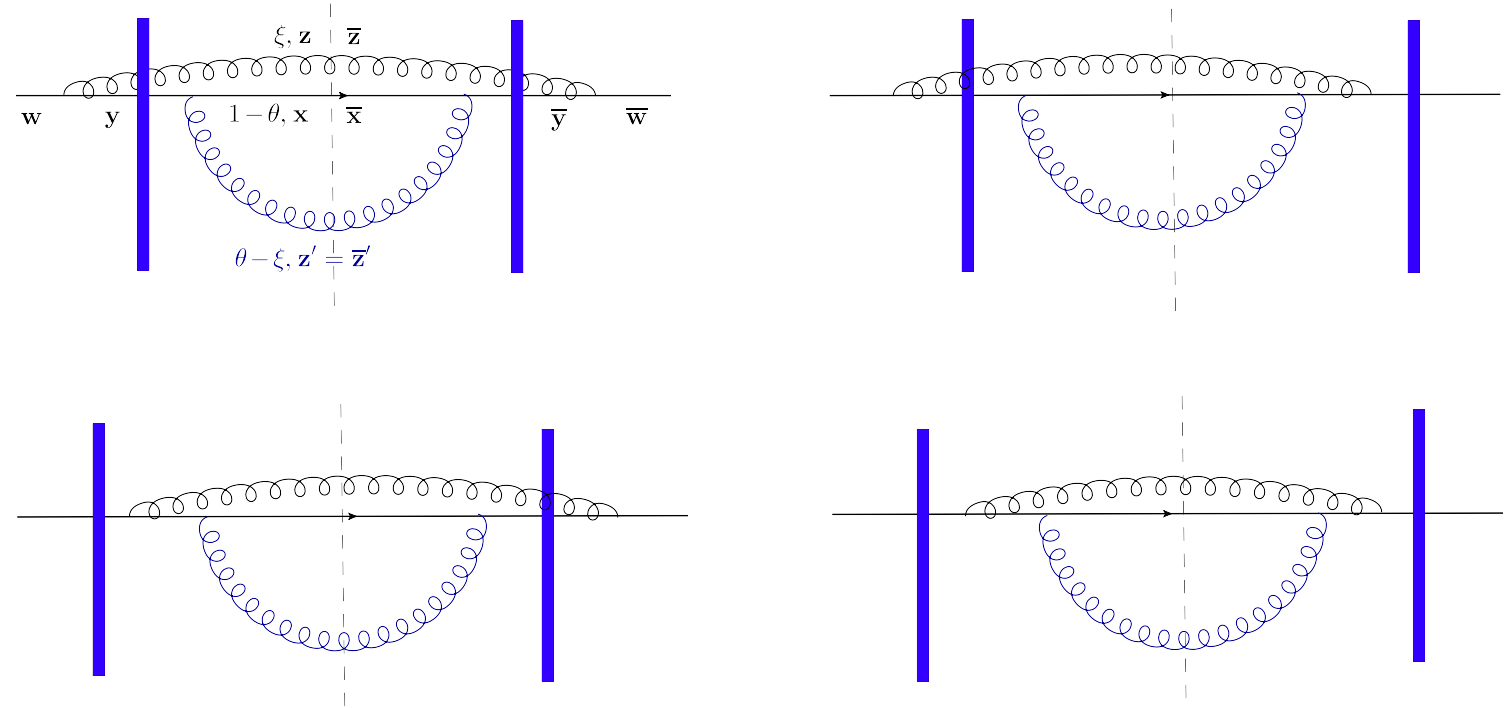

Figure 24. The four graphs surviving in the collinear limit for the second gluon emission (depicted in blue). These graphs are void of final-state interactions.

the DGLAP evolution of the final-state gluon in the leading-order dijet cross-section (2.10). Let us verify that this is indeed the case.

The kernel for the surviving terms has, clearly, the same expression as displayed in eq. (6.1), where the longitudinal momentum fractions must now be evaluated as

$$
\theta=1-\frac{x_{1}}{x_{q}}, \quad \xi=\frac{x_{2}}{x_{q}} \Longrightarrow \xi^{\prime} \equiv \frac{\theta-\xi}{1-\xi}=\frac{x_{q}-\left(x_{1}+x_{2}\right)}{x_{q}-x_{2}} .
$$

The main difference w.r.t. the previous subsection is that the kernel in eq. (6.1) now applies only to the last term within the square brackets in eq. (3.39). After also using $\overline{\boldsymbol{z}}^{\prime}=\boldsymbol{z}^{\prime}$, we find that eq. (6.4) gets replaced by

$$
\begin{aligned}
\frac{d \sigma_{(1) \mathrm{rNL}, 2}^{q A \rightarrow q g+X}}{d p^{+} d^{2} \boldsymbol{p} d k^{+} d^{2} \boldsymbol{k}} \simeq & \frac{4 \alpha_{s} C_{F}}{(2 \pi)^{6}\left(q^{+}\right)^{2}(1-\xi)} P_{q \rightarrow g}(\xi) \\
& \times \int_{\overline{\boldsymbol{x}}, \overline{\boldsymbol{z}}, \boldsymbol{x}, \boldsymbol{z}} e^{-i \boldsymbol{p} \cdot(\boldsymbol{x}-\overline{\boldsymbol{x}})-i \boldsymbol{k} \cdot(\boldsymbol{z}-\overline{\boldsymbol{z}})} \frac{(\boldsymbol{y}-\boldsymbol{z}) \cdot(\overline{\boldsymbol{y}}-\overline{\boldsymbol{z}})}{(\boldsymbol{y}-\boldsymbol{z})^{2}(\overline{\boldsymbol{y}}-\overline{\boldsymbol{z}})^{2}} \\
& \times[\mathcal{Q}(\boldsymbol{y}, \boldsymbol{z}, \overline{\boldsymbol{z}}, \overline{\boldsymbol{y}}) \mathcal{S}(\boldsymbol{z}, \overline{\boldsymbol{z}})-\mathcal{S}(\boldsymbol{y}, \boldsymbol{z}) \mathcal{S}(\boldsymbol{z}, \overline{\boldsymbol{w}})-\mathcal{S}(\boldsymbol{w}, \overline{\boldsymbol{z}}) \mathcal{S}(\overline{\boldsymbol{z}}, \overline{\boldsymbol{y}})+\mathcal{S}(\boldsymbol{w}, \overline{\boldsymbol{w}})] \\
& \times \frac{4 \alpha_{s} C_{F}}{(2 \pi)^{2}} P_{q \rightarrow g}\left(\xi^{\prime}\right) \int_{\boldsymbol{z}^{\prime}} \frac{\left(\boldsymbol{x}-\boldsymbol{z}^{\prime}\right) \cdot\left(\overline{\boldsymbol{x}}-\boldsymbol{z}^{\prime}\right)}{\left(\boldsymbol{x}-\boldsymbol{z}^{\prime}\right)^{2}\left(\overline{\boldsymbol{x}}-\boldsymbol{z}^{\prime}\right)^{2}}
\end{aligned}
$$

For reasons to shortly become clear, it is convenient to consider $z_{1} \equiv 1-\xi^{\prime}$ - the splitting fraction of the measured quark at the second emission vertex - as an independent variable, on the same footing as the external variables $x_{1}$ and $x_{2}$. Then the longitudinal fractions $\xi$ and $x_{q}$ and the transverse coordinate $\boldsymbol{y}$ should be understood as (recall eqs. (3.16) and (6.11))

$$
x_{q}=x_{2}+\frac{x_{1}}{z_{1}}, \quad \xi=\frac{z_{1} x_{2}}{x_{1}+z_{1} x_{2}}, \quad \boldsymbol{y}=z_{1} \boldsymbol{x}+\left(1-z_{1}\right) \boldsymbol{z}^{\prime},
$$

together with a similar expression for $\overline{\boldsymbol{y}}$. 
It is furthermore useful to change two of the integration variables, from $\boldsymbol{x}$ and $\overline{\boldsymbol{x}}$ to $\boldsymbol{y}$ and $\overline{\boldsymbol{y}}$ (this will facilitate the comparison with the LO result in eq. (2.10)). Recalling that $\overline{\boldsymbol{z}}^{\prime}=\boldsymbol{z}^{\prime}$, one finds

$$
\boldsymbol{p} \cdot(\boldsymbol{x}-\overline{\boldsymbol{x}})=\frac{1}{z_{1}} \boldsymbol{p} \cdot(\boldsymbol{y}-\overline{\boldsymbol{y}}), \quad \boldsymbol{x}-\boldsymbol{z}^{\prime}=\frac{1}{z_{1}}\left(\boldsymbol{y}-\boldsymbol{z}^{\prime}\right) .
$$

When expressed in terms of these new variables, $\boldsymbol{w}=(1-\xi) \boldsymbol{y}+\xi \boldsymbol{z}$ becomes independent of $\boldsymbol{z}^{\prime}$, so the integral over $\boldsymbol{z}^{\prime}$ factorizes.

After also convoluting with the initial quark distribution, ${ }^{16}$ as shown in the l.h.s. of eq. (6.8), and changing the respective integration variable from $x_{q}$ to $z_{1}$ according to eq. (6.13), one obtains the following expression for the collinear singularity encoded in this particular NLO correction to the dijet cross-section

$$
\begin{aligned}
\frac{d \sigma_{(1) \mathrm{rNL}, 2}^{p A \rightarrow q g+X}}{d p^{+} d^{2} \boldsymbol{p} d k^{+} d^{2} \boldsymbol{k}} \simeq & \int \frac{d z_{1}}{z_{1}^{3}} x_{q} q_{f}\left(x_{q}, \mu^{2}\right) \frac{4 \alpha_{s} C_{F}}{(2 \pi)^{6}\left(q^{+}\right)^{2}} P_{q \rightarrow g}(\xi) \\
& \times \int_{\overline{\boldsymbol{y}}, \overline{\boldsymbol{z}}, \boldsymbol{y}, \boldsymbol{z}} e^{-i \boldsymbol{p} \cdot(\boldsymbol{y}-\overline{\boldsymbol{y}}) / z_{1}-i \boldsymbol{k} \cdot(\boldsymbol{z}-\overline{\boldsymbol{z}})} \frac{(\boldsymbol{y}-\boldsymbol{z}) \cdot(\overline{\boldsymbol{y}}-\overline{\boldsymbol{z}})}{(\boldsymbol{y}-\boldsymbol{z})^{2}(\overline{\boldsymbol{y}}-\overline{\boldsymbol{z}})^{2}} \\
& \times[\mathcal{Q}(\boldsymbol{y}, \boldsymbol{z}, \overline{\boldsymbol{z}}, \overline{\boldsymbol{y}}) \mathcal{S}(\boldsymbol{z}, \overline{\boldsymbol{z}})-\mathcal{S}(\boldsymbol{y}, \boldsymbol{z}) \mathcal{S}(\boldsymbol{z}, \overline{\boldsymbol{w}})-\mathcal{S}(\boldsymbol{w}, \overline{\boldsymbol{z}}) \mathcal{S}(\overline{\boldsymbol{z}}, \overline{\boldsymbol{y}})+\mathcal{S}(\boldsymbol{w}, \overline{\boldsymbol{w}})] \\
& \times \frac{4 \alpha_{s} C_{F}}{(2 \pi)^{2}} P_{q \rightarrow q}\left(z_{1}\right) \int_{\boldsymbol{z}^{\prime}} \frac{\left(\boldsymbol{y}-\boldsymbol{z}^{\prime}\right) \cdot\left(\overline{\boldsymbol{y}}-\boldsymbol{z}^{\prime}\right)}{\left(\boldsymbol{y}-\boldsymbol{z}^{\prime}\right)^{2}\left(\overline{\boldsymbol{y}}-\boldsymbol{z}^{\prime}\right)^{2}}
\end{aligned}
$$

where we have also used $x_{1} /(1-\xi)=x_{q} z_{1}$ and $P_{q \rightarrow g}\left(1-z_{1}\right)=P_{q \rightarrow q}\left(z_{1}\right)$.

Eq. (6.12) exhibits a factorised structure, as expected: it is the product of the LO dijet cross-section in eq. (2.10) (but for final momenta $p_{1}=p / z_{1}$ and $k$ ) times the probability for an unobserved emission in the final state. This probability contains a collinear singularity similar to that visible in eq. (6.7), which here should be absorbed into the renormalisation of the fragmentation function of the final quark. Specifically, the singular piece of eq. (6.12) can be written as

$$
\left.\frac{d \sigma_{(1) \mathrm{rNLO}, 2}^{p A \rightarrow q+X}}{d p^{+} d^{2} \boldsymbol{p} d k^{+} d^{2} \boldsymbol{k}}\right|_{\text {coll }}=\left.\int \frac{d z_{1}}{z_{1}^{3}} x_{q} q_{f}\left(x_{q}, \mu^{2}\right) \frac{d \sigma_{\mathrm{LO}}^{p A \rightarrow q g+X}}{d^{3} p_{1} d^{3} k}\right|_{p_{1}=p / z_{1}} D_{g / q}\left(z_{1}, \mu^{2}\right),
$$

where

$$
D_{g / q}\left(z_{1}, \mu^{2}\right) \equiv \frac{\alpha_{s} C_{F}}{\pi} P_{q \rightarrow q}\left(z_{1}\right) \ln \frac{\mu^{2}}{\Lambda^{2}} .
$$

is recognised as the contribution of the first step in the DGLAP evolution of the quark-togluon fragmentation function.

\footnotetext{
${ }^{16}$ Strictly speaking, to the NLO accuracy of this calculation, one should evaluate eq. (6.15) with the bare quark distribution $q_{f}^{(0)}\left(x_{q}\right)$, as in eqs. (6.8) and (6.9). We nevertheless insert the renormalised (i.e. DGLAPevolved) distribution $q_{f}\left(x_{q}, \mu^{2}\right)$, for more generality. (This would be generated via a similar treatment of collinear divergences occurring in higher orders of perturbation theory.) Also, following the standard procedure in the literature $[2,21]$, we shall use the same renormalisation scale $\mu^{2}$ for both the parton distributions and the fragmentation functions (see e.g. eq. (6.16) below). This choice is particularly natural when using dimensional regularisation for the 2-dimensional integrals developing collinear divergences, as in ref. [21].
} 

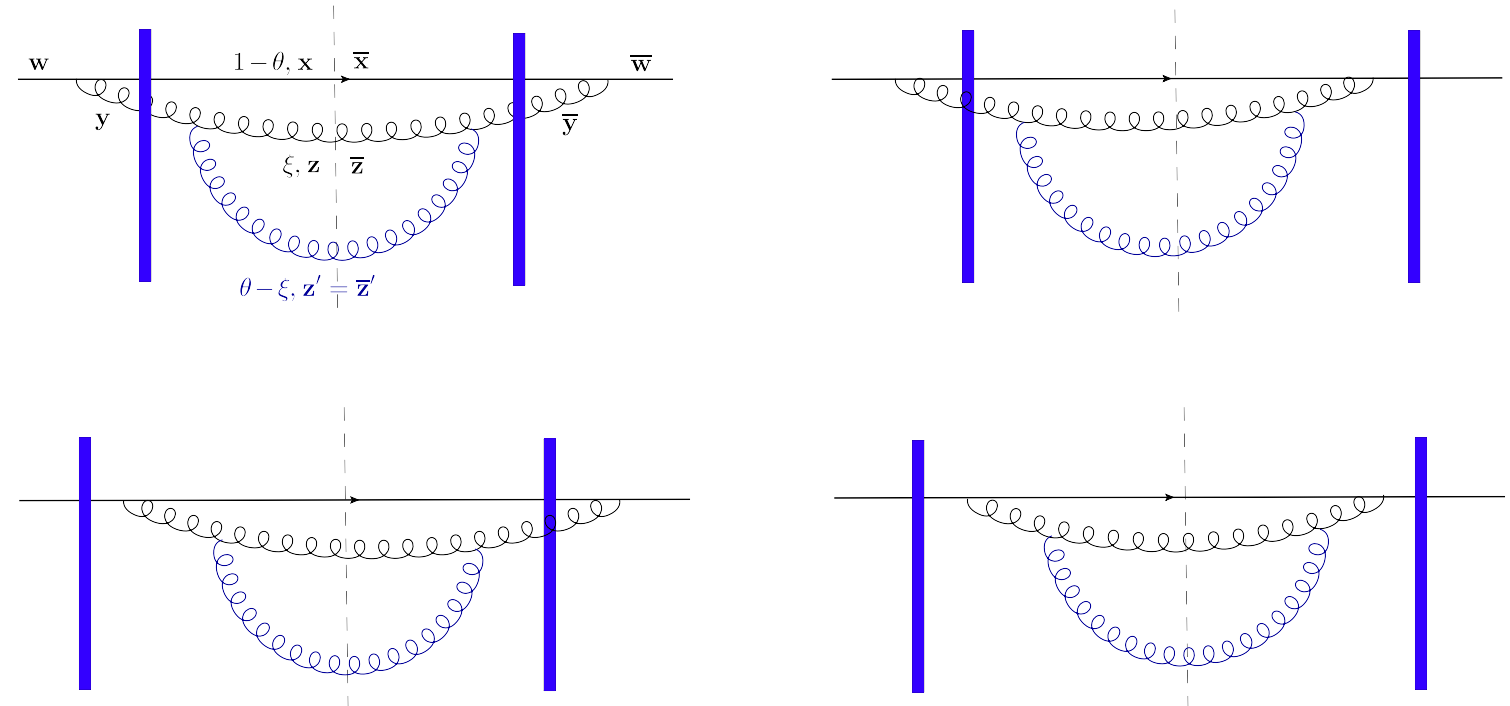

Figure 25. The four graphs surviving in the collinear limit for the $g \rightarrow g g$ splitting (the unmeasured gluon is depicted in blue). These graphs are void of final-state interactions.

To summarise, eqs. (6.15)-(6.17) describe the beginning of the DGLAP evolution of the quark fragmentation function in eq. (2.18). The corresponding evolution of the gluon fragmentation function will be discussed in the next two sections.

\subsection{DGLAP evolution for the final gluon: $g \rightarrow g g$ splitting}

We now consider the collinear limit for the second topology yielding real NLO corrections to quark-gluon production, the one denoted by the subscript (2) in eq. (4.16) and which is illustrated in figure 7 . In this case, a collinear divergence can be generated only by the second emission (see the discussion towards the end of this section), in which case it is associated with the DGLAP evolution of the fragmentation function for the final gluon.

So let us consider the collinear limit for the second emission in figure 7 . This is the limit where the transverse separation $\boldsymbol{Z}=\boldsymbol{z}-\boldsymbol{z}^{\prime}$ between the two final gluons is much larger than the separation $\boldsymbol{R}=\boldsymbol{x}-\boldsymbol{y}$ between the final quark and the intermediate gluon: $\boldsymbol{Z}^{2} \gg \boldsymbol{R}^{2}$. With reference to eq. (3.46), this hierarchy entails two important simplification. The first one refers to the energy denominator and is fully similar to that discussed in the previous section: it implies that the only surviving graphs are the four graphs without finalstate interactions, shown in figure 25. These graphs correspond to the last term within the square brackets in eq. (3.46) - the only one not to be suppressed at large $\boldsymbol{Z}^{2}$. The second simplification refers to the product (3.48) of effective vertices: in eq. (3.46), this product is multiplied by $\boldsymbol{R}^{m} \boldsymbol{Z}^{n} \overline{\boldsymbol{R}}^{p} \overline{\boldsymbol{Z}}^{q}$. When computing the graphs in figure 25, the integral over the coordinate $\boldsymbol{z}^{\prime}$ of the unmeasured gluon $\left(\boldsymbol{z}^{\prime}=\overline{\boldsymbol{z}}^{\prime}\right)$ can be factorised as

$$
\int_{\boldsymbol{z}^{\prime}} \frac{\boldsymbol{Z}^{n} \overline{\boldsymbol{Z}}^{q}}{\boldsymbol{Z}^{2} \overline{\boldsymbol{Z}}^{2}} \simeq \int_{\boldsymbol{z}^{\prime}} \frac{\boldsymbol{Z}^{n} \boldsymbol{Z}^{q}}{\boldsymbol{Z}^{4}}=\frac{\delta^{n q}}{2} \int_{\boldsymbol{z}^{\prime}} \frac{1}{\boldsymbol{Z}^{2}} \simeq \frac{\delta^{n q}}{2} \pi \ln \frac{1}{(\boldsymbol{z}-\overline{\boldsymbol{z}})^{2} \Lambda^{2}}
$$

where the approximate equality holds for the singular piece alone: in the collinear limit at hand, the differences $\boldsymbol{Z}=\boldsymbol{z}-\boldsymbol{z}^{\prime}$ and $\overline{\boldsymbol{Z}}=\overline{\boldsymbol{z}}-\boldsymbol{z}^{\prime}$ can be arbitrarily large, whereas $|\boldsymbol{z}-\overline{\boldsymbol{z}}|$ is 
fixed to a value $\sim 1 / k_{\perp}$ by the Fourier transform for the measured gluon; so, in evaluating the singular piece of (6.18), one can approximate $\boldsymbol{Z} \simeq \bar{Z}$ and at the same time restrict the integral over $\boldsymbol{z}^{\prime}$ to $\boldsymbol{Z}^{2}>(\boldsymbol{z}-\overline{\boldsymbol{z}})^{2}$. The logarithmic divergence at $\boldsymbol{Z}^{2} \rightarrow \infty$ has been regulated by a momentum cutoff $\Lambda^{2}$, as in eq. (6.7).

Eq. (6.18) shows that, for the purpose of extracting the collinear singularity, one can replace $\boldsymbol{Z}^{n} \overline{\boldsymbol{Z}}^{q} \rightarrow(\boldsymbol{Z} \cdot \overline{\boldsymbol{Z}}) \delta^{n q} / 2$ within the integrand of eq. (3.46). This allows us to simplify the tensorial structure of the product of effective vertices (cf. eq. (3.48)):

$$
\begin{aligned}
\Pi_{\lambda_{1} \lambda}^{i j m n} \Pi_{\lambda_{1} \lambda}^{i j p q *} \boldsymbol{R}^{m} \overline{\boldsymbol{R}}^{p}(\boldsymbol{Z} \cdot \overline{\boldsymbol{Z}}) \frac{\delta^{n q}}{2}= & 4 \xi(\theta-\xi) \theta^{2}(1-\theta)^{2}\left[1+(1-\theta)^{2}\right] \\
& \times\left(\frac{1}{\theta^{2}}+\frac{1}{\xi^{2}}+\frac{1}{(\theta-\xi)^{2}}\right)(\boldsymbol{R} \cdot \overline{\boldsymbol{R}})(\boldsymbol{Z} \cdot \overline{\boldsymbol{Z}})
\end{aligned}
$$

As a check, we note that the eikonal limit $\xi \ll \theta$ of this result is consistent with eq. (5.8).

The rest of the calculation is straightforward and yields (see also figure 25):

$$
\begin{aligned}
\frac{d \sigma_{(2) \mathrm{rNLO}, 2}^{q A \rightarrow q g+X}}{d p^{+} d^{2} \boldsymbol{p} d k^{+} d^{2} \boldsymbol{k}} \simeq & \frac{4 \alpha_{s} C_{F}}{(2 \pi)^{6}\left(q^{+}\right)^{2} \theta} P_{q \rightarrow g}(\theta) \\
& \times \int_{\overline{\boldsymbol{x}}, \overline{\boldsymbol{z}}, \boldsymbol{x}, \boldsymbol{z}} e^{-i \boldsymbol{p} \cdot(\boldsymbol{x}-\overline{\boldsymbol{x}})-i \boldsymbol{k} \cdot(\boldsymbol{z}-\overline{\boldsymbol{z}})} \frac{(\boldsymbol{x}-\boldsymbol{y}) \cdot(\overline{\boldsymbol{x}}-\overline{\boldsymbol{y}})}{(\boldsymbol{x}-\boldsymbol{y})^{2}(\overline{\boldsymbol{x}}-\overline{\boldsymbol{y}})^{2}} \\
& \times[\mathcal{Q}(\boldsymbol{x}, \boldsymbol{y}, \overline{\boldsymbol{y}}, \overline{\boldsymbol{x}}) \mathcal{S}(\boldsymbol{y}, \overline{\boldsymbol{y}})-\mathcal{S}(\boldsymbol{x}, \boldsymbol{y}) \mathcal{S}(\boldsymbol{y}, \overline{\boldsymbol{w}})-\mathcal{S}(\boldsymbol{w}, \overline{\boldsymbol{y}}) \mathcal{S}(\overline{\boldsymbol{y}}, \overline{\boldsymbol{x}})+\mathcal{S}(\boldsymbol{w}, \overline{\boldsymbol{w}})] \\
& \times \frac{4 \alpha_{s} N_{c}}{(2 \pi)^{2}} P_{g \rightarrow g}(\xi / \theta) \int_{\boldsymbol{z}^{\prime}} \frac{\left(\boldsymbol{z}-\boldsymbol{z}^{\prime}\right) \cdot\left(\overline{\boldsymbol{z}}-\boldsymbol{z}^{\prime}\right)}{\left(\boldsymbol{z}-\boldsymbol{z}^{\prime}\right)^{2}\left(\overline{\boldsymbol{z}}-\boldsymbol{z}^{\prime}\right)^{2}}
\end{aligned}
$$

where $z_{2} \equiv \xi / \theta$ is the splitting fraction of the measured gluon and

$$
P_{g \rightarrow g}(z) \equiv \frac{[1-z(1-z)]^{2}}{z(1-z)}
$$

is the gluon-to-gluon LO DGLAP splitting function. It is understood that only the logarithmic collinear singularity is properly encoded in eq. (6.20), but not also the constant term under the logarithm.

At this stage, it is convenient to express all the longitudinal fractions in terms of $x_{1}$, $x_{2}$ and $z_{2}$ :

$$
x_{q}=x_{1}+\frac{x_{2}}{z_{2}}, \quad \theta=1-\frac{x_{1}}{x_{q}}=\frac{x_{2}}{x_{2}+z_{1} x_{1}}, \quad \xi=\theta z_{2} .
$$

Also, we make a change of integration variables, from $\boldsymbol{z}$ and $\overline{\boldsymbol{z}}$ to $\boldsymbol{y}$ and $\overline{\boldsymbol{y}}$, by using

$$
\boldsymbol{y}=z_{2} \boldsymbol{z}+\left(1-z_{2}\right) \boldsymbol{z}^{\prime} \Longrightarrow \boldsymbol{z}-\overline{\boldsymbol{z}}=\frac{1}{z_{2}}(\boldsymbol{y}-\overline{\boldsymbol{y}}), \quad \boldsymbol{z}-\boldsymbol{z}^{\prime}=\frac{1}{z_{2}}\left(\boldsymbol{y}-\boldsymbol{z}^{\prime}\right)
$$

together with $\boldsymbol{w}=(1-\theta) \boldsymbol{x}+\theta \boldsymbol{y}$. 
We finally add the convolution with the quark distribution function $q_{f}\left(x_{q}, \mu^{2}\right)$, but use $z_{2}$ rather than $x_{q}$ as an integration variable. Putting all that together one finds

$$
\begin{aligned}
\frac{d \sigma_{(2) \mathrm{rNLO}, 2}^{p A \rightarrow q g+X}}{d p^{+} d^{2} \boldsymbol{p} d k^{+} d^{2} \boldsymbol{k}} \simeq & \int \frac{d z_{2}}{z_{2}^{3}} x_{q} q_{f}\left(x_{q}, \mu^{2}\right) \frac{4 \alpha_{s} C_{F}}{(2 \pi)^{6}\left(q^{+}\right)^{2}} P_{q \rightarrow g}(\theta) \\
& \times \int_{\overline{\boldsymbol{x}}, \overline{\boldsymbol{y}}, \boldsymbol{x}, \boldsymbol{y}} e^{-i \boldsymbol{p} \cdot(\boldsymbol{x}-\overline{\boldsymbol{x}})-i \boldsymbol{k} \cdot(\boldsymbol{y}-\overline{\boldsymbol{y}}) / z_{2}} \frac{(\boldsymbol{x}-\boldsymbol{y}) \cdot(\overline{\boldsymbol{x}}-\overline{\boldsymbol{y}})}{(\boldsymbol{x}-\boldsymbol{y})^{2}(\overline{\boldsymbol{x}}-\overline{\boldsymbol{y}})^{2}} \\
& \times[\mathcal{Q}(\boldsymbol{x}, \boldsymbol{y}, \overline{\boldsymbol{y}}, \overline{\boldsymbol{x}}) \mathcal{S}(\boldsymbol{y}, \overline{\boldsymbol{y}})-\mathcal{S}(\boldsymbol{x}, \boldsymbol{y}) \mathcal{S}(\boldsymbol{y}, \overline{\boldsymbol{w}})-\mathcal{S}(\boldsymbol{w}, \overline{\boldsymbol{y}}) \mathcal{S}(\overline{\boldsymbol{y}}, \overline{\boldsymbol{x}})+\mathcal{S}(\boldsymbol{w}, \overline{\boldsymbol{w}})] \\
& \times \frac{4 \alpha_{s} N_{c}}{(2 \pi)^{2}} P_{g \rightarrow g}\left(z_{2}\right) \int_{\boldsymbol{z}^{\prime}} \frac{\left(\boldsymbol{y}-\boldsymbol{z}^{\prime}\right) \cdot\left(\overline{\boldsymbol{y}}-\boldsymbol{z}^{\prime}\right)}{\left(\boldsymbol{y}-\boldsymbol{z}^{\prime}\right)^{2}\left(\overline{\boldsymbol{y}}-\boldsymbol{z}^{\prime}\right)^{2}}
\end{aligned}
$$

This is analog to eq. (6.15) from the previous section and its subsequent discussion is also similar: the collinear divergence can be absorbed in one step of the DGLAP evolution of the gluon-to-gluon fragmentation function:

$$
D_{g / g}\left(z_{2}, \mu^{2}\right) \equiv \frac{\alpha_{s} N_{c}}{\pi} P_{g \rightarrow g}\left(z_{2}\right) \ln \frac{\mu^{2}}{\Lambda^{2}}
$$

To conclude this section, let us explain why the first emission cannot contribute a collinear divergence for this particular topology. The first emitted gluon is an intermediate gluon, which decays into two other gluons prior the final state. One of these daughter gluons is measured in the final state $(\overline{\boldsymbol{z}} \neq \boldsymbol{z})$, and the other one is not $\left(\overline{\boldsymbol{z}}^{\prime}=\boldsymbol{z}^{\prime}\right)$. Hence the coordinates, $\boldsymbol{y}$ and $\overline{\boldsymbol{y}}$, of the intermediate gluon are different in the DA and the CCA, respectively (as also manifest in eq. (6.20)). Hence, the would-be collinear limit for the first emission, that is, $\boldsymbol{R}^{2} \gg \boldsymbol{Z}^{2}$, cannot generate a collinear divergence in the integral over the transverse coordinate $\boldsymbol{z}^{\prime}$ of the unmeasured daughter gluon.

One can similarly understand that the interference graphs responsible for the piece denoted by the subscript (3) in eq. (4.16) (see figure 11) do not generate collinear divergences either. This is consistent with the fact that the LO DGLAP evolution admits a probabilistic picture and could not accommodate such interference effects.

\subsection{DGLAP evolution for the final gluon: $g \rightarrow q \bar{q}$ splitting}

As a final exemple for the DGLAP evolution of the quark-gluon cross-section, let us briefly consider the case where the gluon undergoes a collinear splitting into a quark-antiquark pair, thus contributing to the $q q \bar{q}$ final state. With reference to eq. (3.27) for the respective cross-section, this situation corresponds to the case where $\boldsymbol{Z}^{2} \gg \boldsymbol{R}^{2}$ and one of the daughter fermions of the gluon decay - say the antiquark with longitudinal fraction $\theta-\xi$ (see figure 8) is not measured $\left(\overline{\boldsymbol{z}}^{\prime}=\boldsymbol{z}^{\prime}\right)$. Clearly, the treatment of the $g \rightarrow q \bar{q}$ collinear splitting is entirely analog to that of the $g \rightarrow g g$ splitting discussed in the previous section. Once again, the surviving graphs are the four graphs corresponding to the last term in eq. (3.27), which contain no final-state interactions. (The respective $S$-matrix structure has been already exhibited in eq. (3.36).) And the product of effective vertices which enters the kernel (3.30) can be simplified - in so far as the collinear divergence is concerned — by 
using the analog of eq. (6.18). This yields (recall also eq. (3.31))

$$
\Phi_{\lambda_{3} \lambda_{2} \lambda_{1} \lambda}^{i j} \Phi_{\lambda_{3} \lambda_{2} \lambda_{1} \lambda}^{m n} \boldsymbol{R}^{i} \overline{\boldsymbol{R}}^{m}(\boldsymbol{Z} \cdot \overline{\boldsymbol{Z}}) \frac{\delta^{j n}}{2}=8(1-\theta)^{2}\left(1+(1-\theta)^{2}\right)\left((\theta-\xi)^{2}+\xi^{2}\right)(\boldsymbol{R} \cdot \overline{\boldsymbol{R}})(\boldsymbol{Z} \cdot \overline{\boldsymbol{Z}}) .
$$

Inserting this into eq. (3.27) and integrating over $k^{3}$, one finds (we relabel the final momenta as $p$ and $k$ for the two measured quark, with $p$ referring to the leading quark)

$$
\begin{aligned}
\frac{d \sigma_{\mathrm{rNLO}, 2}^{q A \rightarrow q q+X}}{d p^{+} d^{2} \boldsymbol{p} d k^{+} d^{2} \boldsymbol{k}} \simeq & \frac{4 \alpha_{s} C_{F}}{(2 \pi)^{6}\left(q^{+}\right)^{2} \theta} P_{q \rightarrow g}(\theta) \\
& \times \int_{\overline{\boldsymbol{x}}, \overline{\boldsymbol{z}}, \boldsymbol{x}, \boldsymbol{z}} e^{-i \boldsymbol{p} \cdot(\boldsymbol{x}-\overline{\boldsymbol{x}})-i \boldsymbol{k} \cdot(\boldsymbol{z}-\overline{\boldsymbol{z}})} \frac{(\boldsymbol{x}-\boldsymbol{y}) \cdot(\overline{\boldsymbol{x}}-\overline{\boldsymbol{y}})}{(\boldsymbol{x}-\boldsymbol{y})^{2}(\overline{\boldsymbol{x}}-\overline{\boldsymbol{y}})^{2}} \\
& \times[\mathcal{Q}(\boldsymbol{x}, \boldsymbol{y}, \overline{\boldsymbol{y}}, \overline{\boldsymbol{x}}) \mathcal{S}(\boldsymbol{y}, \overline{\boldsymbol{y}})-\mathcal{S}(\boldsymbol{x}, \boldsymbol{y}) \mathcal{S}(\boldsymbol{y}, \overline{\boldsymbol{w}})-\mathcal{S}(\boldsymbol{w}, \overline{\boldsymbol{y}}) \mathcal{S}(\overline{\boldsymbol{y}}, \overline{\boldsymbol{x}})+\mathcal{S}(\boldsymbol{w}, \overline{\boldsymbol{w}})] \\
& \times \frac{4 \alpha_{s} N_{f}}{(2 \pi)^{2}} P_{g \rightarrow q}(\xi / \theta) \int_{\boldsymbol{z}^{\prime}} \frac{\left(\boldsymbol{z}-\boldsymbol{z}^{\prime}\right) \cdot\left(\overline{\boldsymbol{z}}-\boldsymbol{z}^{\prime}\right)}{\left(\boldsymbol{z}-\boldsymbol{z}^{\prime}\right)^{2}\left(\overline{\boldsymbol{z}}-\boldsymbol{z}^{\prime}\right)^{2}}
\end{aligned}
$$

which also involves the LO DGLAP splitting function for the $g \rightarrow q \bar{q}$ splitting:

$$
P_{g \rightarrow q}(z) \equiv \frac{z^{2}+(1-z)^{2}}{2} .
$$

The similarity with eq. (6.20) is manifest. Once again, the collinear singularity encoded in the above integral over $\boldsymbol{z}^{\prime}$ is reabsorbed into a contribution to one-step in the DGLAP evolution of the fragmentation function for gluon fragmenting into quarks:

$$
D_{q / g}\left(z, \mu^{2}\right) \equiv \frac{\alpha_{s} N_{f}}{\pi} P_{g \rightarrow q}(z) \ln \frac{\mu^{2}}{\Lambda^{2}} .
$$

\section{Acknowledgments}

We would like to thank G. Beuf, T. Lappi, M. Lublinsky, and A. H. Mueller for useful discussions. The work of E.I. is supported in part by the Agence Nationale de la Recherche project ANR-16-CE31-0019-01. The work of Y.M. was in part supported by the 2016-2017 Chateaubriand fellowship of the French embassy in Israel. This work is supported under the European Union's Horizon 2020 research and innovation programme by the European Research Council (ERC), grant agreement No. ERC-2015-CoG-681707 (Y.M. since 2018) and by the STRONG-2020 project (grant agreement No 824093). The content of this article does not reflect the official opinion of the European Union and responsibility for the information and views expressed therein lies entirely with the authors.

\section{A Definitions of states and field quantization}

In this appendix, we summarise our conventions for the field quantisation and the definition of the bare Fock states. More details on the light-cone wavefunction formalism, including the complete expression of the QCD Hamiltonian in the light-cone gauge, can be found in appendix $\mathbf{A}$ of ref. [1]. 
Quantisation of the fields is performed in usual manner introducing creation/annihilation operators and imposing commutation (anti-commutation) relations among them. For the gauge fields we shall use the following expansion:

$$
A_{i}^{a}(x)=\int_{0}^{\infty} \frac{d k^{+}}{2 \pi} \int \frac{d^{2} \boldsymbol{k}}{(2 \pi)^{2}} \frac{1}{\sqrt{2 k^{+}}}\left(a_{i}^{a}\left(k^{+}, \boldsymbol{k}\right) e^{-i k \cdot x}+a_{i}^{a \dagger}\left(k^{+}, \boldsymbol{k}\right) e^{i k \cdot x}\right) .
$$

The creation and annihilation operators obey the bosonic algebra:

$$
\left[a_{i}^{a}\left(k^{+}, \boldsymbol{k}\right), a_{j}^{b \dagger}\left(p^{+}, \boldsymbol{p}\right)\right]=(2 \pi)^{3} \delta^{a b} \delta_{i j} \delta\left(k^{+}-p^{+}\right) \delta^{(2)}(\boldsymbol{k}-\boldsymbol{p}) .
$$

Transforming to coordinate space,

$$
a_{i}^{a}\left(k^{+}, \boldsymbol{k}\right)=\int d^{2} \boldsymbol{z} \mathrm{e}^{-i \boldsymbol{k} \cdot \boldsymbol{z}} a_{i}^{a}\left(k^{+}, \boldsymbol{z}\right),
$$

the commutation relation becomes:

$$
\left[a_{i}^{a}\left(k^{+}, \boldsymbol{x}\right), a_{j}^{b \dagger}\left(p^{+}, \boldsymbol{y}\right)\right]=2 \pi \delta^{a b} \delta_{i j} \delta\left(k^{+}-p^{+}\right) \delta^{(2)}(\boldsymbol{x}-\boldsymbol{y}) .
$$

The quark fields can be expanded by the following expression:

$$
\psi_{+}^{\alpha}(x)=\chi_{\lambda} \int_{0}^{\infty} \frac{d k^{+}}{2 \pi} \int \frac{d^{2} \boldsymbol{k}}{(2 \pi)^{2}} \frac{1}{\sqrt{2}}\left(b_{\lambda}^{\alpha}\left(k^{+}, \boldsymbol{k}\right) e^{-i k \cdot x}+d_{\lambda}^{\alpha \dagger}\left(k^{+}, \boldsymbol{k}\right) e^{i k \cdot x}\right) .
$$

The polarisation vectors are:

$$
\begin{array}{ll}
\chi_{+\frac{1}{2}}=\left(\begin{array}{l}
1 \\
0
\end{array}\right), & \chi_{-\frac{1}{2}}=\left(\begin{array}{l}
0 \\
1
\end{array}\right), \\
\chi_{\lambda} \chi_{\lambda}^{\dagger}=I, & \chi_{\lambda}^{\dagger} \chi_{\lambda}=2, \quad \chi_{\lambda_{1}}^{\dagger} I \chi_{\lambda_{2}}=\delta_{\lambda_{1} \lambda_{2}}, \quad \chi_{\lambda_{1}}^{\dagger} \sigma^{3} \chi_{\lambda_{2}}=2 \lambda_{1} \delta_{\lambda_{1} \lambda_{2}} .
\end{array}
$$

The anti-commutation relations:

$$
\begin{aligned}
\left\{b_{\lambda_{1}}^{\alpha}\left(k^{+}, \boldsymbol{k}\right), b_{\lambda_{2}}^{\beta \dagger}\left(p^{+}, \boldsymbol{p}\right)\right\} & =\left\{d_{\lambda_{1}}^{\alpha}\left(k^{+}, \boldsymbol{k}\right), d_{\lambda_{2}}^{\beta \dagger}\left(p^{+}, \boldsymbol{p}\right)\right\} \\
& =(2 \pi)^{3} \delta_{\lambda_{1} \lambda_{2}} \delta^{\alpha \beta} \delta^{(2)}(\boldsymbol{k}-\boldsymbol{p}) \delta\left(k^{+}-p^{+}\right) .
\end{aligned}
$$

Transforming the fields to coordinate space a la (A.3):

$$
\begin{aligned}
\left\{b_{\lambda_{1}}^{\alpha}\left(k^{+}, \boldsymbol{x}\right), b_{\lambda_{2}}^{\beta \dagger}\left(p^{+}, \boldsymbol{y}\right)\right\} & =\left\{d_{\lambda_{1}}^{\alpha}\left(k^{+}, \boldsymbol{x}\right), d_{\lambda_{2}}^{\beta \dagger}\left(p^{+}, \boldsymbol{y}\right)\right\} \\
& =2 \pi \delta_{\lambda_{1} \lambda_{2}} \delta^{\alpha \beta} \delta^{(2)}(\boldsymbol{x}-\boldsymbol{y}) \delta\left(k^{+}-p^{+}\right) .
\end{aligned}
$$

The multi-parton bare Fock states are obtained by acting with the relevant creation operators on the bare vacuum state. In this paper, we use both the 3-momentum representation $k=\left(k^{+}, \boldsymbol{k}\right)$ and the mixed representation $\left(k^{+}, \boldsymbol{x}\right)$, as obtained via the Fourier transform from transverse momenta to transverse coordinates. Let us present here a few representative examples.

- The bare vacuum state $|0\rangle$. This state obeys the following conditions:

$$
a_{i}^{a}\left(q^{+}, \boldsymbol{q}\right)|0\rangle=b_{\lambda}^{\alpha}\left(q^{+}, \boldsymbol{q}\right)|0\rangle=d_{\lambda}^{\alpha}\left(q^{+}, \boldsymbol{q}\right)|0\rangle=0 .
$$


- The bare quark state. In momentum space, this state is constructed as

$$
\left|q_{\lambda}^{\alpha}\left(k^{+}, \boldsymbol{k}\right)\right\rangle \equiv b_{\lambda}^{\alpha \dagger}\left(k^{+}, \boldsymbol{k}\right)|0\rangle .
$$

its scalar product is normalized as follows:

$$
\left\langle q_{\lambda_{2}}^{\beta}\left(p^{+}, \boldsymbol{p}\right) \mid q_{\lambda_{1}}^{\alpha}\left(k^{+}, \boldsymbol{k}\right)\right\rangle=(2 \pi)^{3} \delta^{\alpha \beta} \delta_{\lambda_{1} \lambda_{2}} \delta^{(2)}(\boldsymbol{k}-\boldsymbol{p}) \delta\left(k^{+}-p^{+}\right) .
$$

The mixed representation of the bare quark state is obtained as

$$
\left|q_{\lambda}^{\alpha}\left(k^{+}, \boldsymbol{x}\right)\right\rangle \equiv \int \frac{d^{2} \boldsymbol{k}}{(2 \pi)^{2}} \mathrm{e}^{-i \boldsymbol{x} \cdot \boldsymbol{k}}\left|q_{\lambda}^{\alpha}\left(k^{+}, \boldsymbol{k}\right)\right\rangle=b_{\lambda}^{\alpha \dagger}\left(k^{+}, \boldsymbol{x}\right)|0\rangle,
$$

and the dot product reads

$$
\left\langle q_{\lambda_{2}}^{\beta}\left(p^{+}, \boldsymbol{y}\right) \mid q_{\lambda_{1}}^{\alpha}\left(k^{+}, \boldsymbol{x}\right)\right\rangle=2 \pi \delta^{\alpha \beta} \delta_{\lambda_{1} \lambda_{2}} \delta^{(2)}(\boldsymbol{x}-\boldsymbol{y}) \delta\left(k^{+}-p^{+}\right) .
$$

- The bare quark-gluon state. In momentum space, this state reads

$$
\left|q_{\lambda}^{\alpha}\left(p^{+}, \boldsymbol{p}\right) g_{i}^{a}\left(k^{+}, \boldsymbol{k}\right)\right\rangle \equiv b_{\lambda}^{\alpha \dagger}\left(p^{+}, \boldsymbol{p}\right) a_{i}^{a \dagger}\left(k^{+}, \boldsymbol{k}\right)|0\rangle .
$$

The mixed representation of this state reads as follows:

$$
\begin{aligned}
\left|q_{\lambda}^{\alpha}\left(p^{+}, \boldsymbol{x}\right) g_{i}^{a}\left(k^{+}, \boldsymbol{y}\right)\right\rangle & \equiv \int \frac{d^{2} \boldsymbol{p}}{(2 \pi)^{2}} \frac{d^{2} \boldsymbol{k}}{(2 \pi)^{2}} \mathrm{e}^{-i \boldsymbol{x} \cdot \boldsymbol{p}-i \boldsymbol{y} \cdot \boldsymbol{k}}\left|q_{\lambda}^{\alpha}\left(p^{+}, \boldsymbol{p}\right) g_{i}^{a}\left(k^{+}, \boldsymbol{k}\right)\right\rangle= \\
& =b_{\lambda}^{\alpha \dagger}\left(p^{+}, \boldsymbol{x}\right) a_{i}^{a \dagger}\left(k^{+}, \boldsymbol{y}\right)|0\rangle .
\end{aligned}
$$

Let us finally present our definitions for the number density operators for bare partons; they read as follows (for quarks, antiquarks, and gluons, respectively):

$$
\begin{aligned}
\hat{\mathcal{N}}_{q}\left(p^{+}, \boldsymbol{p}\right) & \equiv \frac{1}{(2 \pi)^{3}} b_{\lambda}^{\alpha \dagger}\left(p^{+}, \boldsymbol{p}\right) b_{\lambda}^{\alpha}\left(p^{+}, \boldsymbol{p}\right)=\frac{1}{(2 \pi)^{3}} \int_{\overline{\boldsymbol{x}}, \boldsymbol{x}} e^{i \boldsymbol{p} \cdot(\overline{\boldsymbol{x}}-\boldsymbol{x})} b_{\lambda}^{\alpha \dagger}\left(p^{+}, \overline{\boldsymbol{x}}\right) b_{\lambda}^{\alpha}\left(p^{+}, \boldsymbol{x}\right), \\
\hat{\mathcal{N}}_{\bar{q}}(p) & \equiv \frac{1}{(2 \pi)^{3}} d_{\lambda}^{\alpha \dagger}(p) d_{\lambda}^{\alpha}(p)=\frac{1}{(2 \pi)^{3}} \int_{\overline{\boldsymbol{x}}, \boldsymbol{x}} e^{i \boldsymbol{p} \cdot(\overline{\boldsymbol{x}}-\boldsymbol{x})} d_{\lambda}^{\alpha \dagger}\left(p^{+}, \overline{\boldsymbol{x}}\right) d_{\lambda}^{\alpha}\left(p^{+}, \boldsymbol{x}\right) \\
\hat{\mathcal{N}}_{g}(k) & \equiv \frac{1}{(2 \pi)^{3}} a_{i}^{a \dagger}(k) a_{i}^{a}(k)=\frac{1}{(2 \pi)^{3}} \int_{\overline{\boldsymbol{z}}, \boldsymbol{z}} e^{i \boldsymbol{k} \cdot(\overline{\boldsymbol{z}}-\boldsymbol{z})} a_{i}^{a \dagger}\left(k^{+}, \overline{\boldsymbol{z}}\right) a_{i}^{a}\left(k^{+}, \boldsymbol{z}\right)
\end{aligned}
$$

\section{B Matrix elements}

In terms of the Fock space operators introduced in the previous appendix, the free QCD Hamiltonian takes the following form,

$$
\begin{aligned}
H_{0}= & \int_{0}^{\infty} \frac{d k^{+}}{2 \pi} \int \frac{d^{2} \boldsymbol{k}}{(2 \pi)^{2}} \frac{\boldsymbol{k}^{2}}{2 k^{+}}\left(a_{i}^{a \dagger}\left(k^{+}, \boldsymbol{k}\right) a_{i}^{a}\left(k^{+}, \boldsymbol{k}\right)\right. \\
& \left.+b_{\lambda}^{\alpha \dagger}\left(k^{+}, \boldsymbol{k}\right) b_{\lambda}^{\alpha}\left(k^{+}, \boldsymbol{k}\right)-d_{\lambda}^{\alpha}\left(k^{+}, \boldsymbol{k}\right) d_{\lambda}^{\alpha \dagger}\left(k^{+}, \boldsymbol{k}\right)\right),
\end{aligned}
$$

which in particular shows that the dispersion relation for free quarks and gluons is $E_{k}=$ $\frac{k^{2}}{2 k^{+}}$. We shall not write the corresponding expression for the interaction piece of the Hamiltonian, as this is not needed in full generality for the purposes of this paper. Rather, we show only those matrix elements which enter the computation of the outgoing state in section 3.1: 
- Emission of a second gluon from the quark state

$$
\begin{aligned}
& \left\langle q_{\lambda_{2}}^{\beta}(u) g_{j}^{b}(t) g_{l}^{c}(p)\left|\mathrm{H}_{q \rightarrow q g}\right| q_{\lambda_{1}}^{\alpha}(s) g_{i}^{a}(k)\right\rangle \\
& =\frac{g t_{\beta \alpha}^{c} \delta^{a b} \delta_{i j}}{2 \sqrt{2 p^{+}}} \chi_{\lambda_{2}}^{\dagger}\left[\frac{2 \boldsymbol{p}^{l}}{p^{+}}-\frac{\sigma \cdot \boldsymbol{u}}{u^{+}} \sigma^{l}-\sigma^{l} \frac{\sigma \cdot \boldsymbol{s}}{s^{+}}\right] \chi_{\lambda_{1}}(2 \pi)^{6} \delta^{(3)}(k-t) \delta^{(3)}(s-u-p) \\
& \quad+\frac{g t_{\beta \alpha}^{b} \delta^{a c} \delta_{i l}}{2 \sqrt{2 t^{+}}} \chi_{\lambda_{2}}^{\dagger}\left[\frac{2 \boldsymbol{t}^{j}}{t^{+}}-\frac{\sigma \cdot \boldsymbol{u}}{u^{+}} \sigma^{j}-\sigma^{j} \frac{\sigma \cdot \boldsymbol{s}}{s^{+}}\right] \chi_{\lambda_{1}}(2 \pi)^{6} \delta^{(3)}(k-p) \delta^{(3)}(s-u-t) .
\end{aligned}
$$

- Gluon splits into quark and antiquark pair

$$
\begin{aligned}
& \left\langle\bar{q}_{\lambda_{4}}^{\epsilon}(u) q_{\lambda_{3}}^{\delta}(t) q_{\lambda_{2}}^{\gamma}(p)\left|\mathrm{H}_{g \rightarrow q q}\right| q_{\lambda_{1}}^{\beta}(s) g_{i}^{a}(k)\right\rangle \\
& =\frac{g t_{\gamma \epsilon}^{a} \delta^{\beta \delta} \delta_{\lambda_{1} \lambda_{3}}}{2 \sqrt{2 k^{+}}} \chi_{\lambda_{2}}^{\dagger}\left[\frac{2 \boldsymbol{k}^{i}}{k^{+}}-\frac{\sigma \cdot \boldsymbol{p}}{p^{+}} \sigma^{i}-\sigma^{i} \frac{\sigma \cdot \boldsymbol{u}}{u^{+}}\right] \chi_{\lambda_{4}}(2 \pi)^{6} \delta^{(3)}(s-t) \delta^{(3)}(k-p-u) \\
& \quad+\frac{g t_{\delta \epsilon}^{a} \delta^{\beta \gamma} \delta_{\lambda_{1} \lambda_{2}}}{2 \sqrt{2 k^{+}}} \chi_{\lambda_{3}}^{\dagger}\left[\frac{2 \boldsymbol{k}^{i}}{k^{+}}-\frac{\sigma \cdot \boldsymbol{t}}{t^{+}} \sigma^{i}-\sigma^{i} \frac{\sigma \cdot \boldsymbol{u}}{u^{+}}\right] \chi_{\lambda_{4}}(2 \pi)^{6} \delta^{(3)}(s-p) \delta^{(3)}(k-t-u) .
\end{aligned}
$$

- Triple gluon interaction in presence of a quark

$$
\begin{aligned}
& \left\langle q_{\lambda_{2}}^{\beta}(t) g_{l}^{c}(q) g_{n}^{b}(p)\left|\mathrm{H}_{g \rightarrow g g}\right| q_{\lambda_{1}}^{\alpha}(s) g_{m}^{a}(k)\right\rangle \\
& =\frac{i g f^{a b c} \delta^{\alpha \beta} \delta_{\lambda_{1} \lambda_{2}}}{2 \sqrt{2 k^{+} p^{+} q^{+}}}(2 \pi)^{6} \delta^{(3)}(s-t) \delta^{(3)}(k-p-q) \\
& \quad \times\left[\left(\boldsymbol{p}^{m}-\boldsymbol{q}^{m}+\frac{q^{+}-p^{+}}{k^{+}} \boldsymbol{k}^{m}\right) \delta_{n l}+\left(\boldsymbol{k}^{n}+\boldsymbol{q}^{n}-\frac{k^{+}+q^{+}}{p^{+}} \boldsymbol{p}^{n}\right) \delta_{m l}\right. \\
& \left.\quad+\left(\frac{k^{+}+p^{+}}{q^{+}} \boldsymbol{q}^{l}-\boldsymbol{p}^{l}-\boldsymbol{k}^{l}\right) \delta_{m n}\right] .
\end{aligned}
$$

- Quark produces quark and antiquark pair instantaneously

$$
\left\langle q_{\lambda_{2}}^{\beta}(k) \bar{q}_{\lambda_{3}}^{\gamma}(q) q_{\lambda_{4}}^{\delta}(s)\left|\mathrm{H}_{q \rightarrow q q q}\right| q_{\lambda_{1}}^{\alpha}(p)\right\rangle=\frac{g^{2} t_{\beta \alpha}^{a} t_{\delta \gamma}^{a}}{\left(q^{+}+s^{+}\right)^{2}} \chi_{\lambda_{4}}^{\dagger} \chi_{\lambda_{3}} \chi_{\lambda_{2}}^{\dagger} \chi_{\lambda_{1}}(2 \pi)^{3} \delta(k-p+q+s) .
$$

- Instantaneous emission of a two gluons from the quark state (inst. q)

$$
\begin{aligned}
& \left\langle q_{\lambda_{2}}^{\gamma}(u) g_{j}^{b}(t) g_{l}^{c}(p)\left|\mathrm{H}_{q \rightarrow q g g}^{\text {inst } q}\right| q_{\lambda_{1}}^{\alpha}(s)\right\rangle \\
& \quad=\frac{g^{2}}{\sqrt{t^{+} p^{+}}}\left(\frac{t_{\gamma \beta}^{b} t_{\beta \alpha}^{c}}{s^{+}-p^{+}} \chi_{\lambda_{2}}^{\dagger} \sigma_{j} \sigma_{l} \chi_{\lambda_{1}}+\frac{t_{\gamma \beta}^{c} t_{\beta \alpha}^{b}}{s^{+}-t^{+}} \chi_{\lambda_{2}}^{\dagger} \sigma_{l} \sigma_{j} \chi_{\lambda_{1}}\right)(2 \pi)^{3} \delta(t+p+u-s) .
\end{aligned}
$$


- Instantaneous emission of two gluons from the quark state (inst. g)

$$
\begin{aligned}
& \left\langle q_{\lambda_{2}}^{\beta}(s) g_{j}^{c}(p) g_{i}^{b}(k)\left|\mathrm{H}_{q \rightarrow q g g}^{\text {inst g }}\right| q_{\lambda_{1}}^{\alpha}(q)\right\rangle \\
& \quad=\frac{i g^{2} f^{a b c} t_{\beta \alpha}^{a}\left(p^{+}-k^{+}\right)}{2 \sqrt{k^{+} p^{+}}\left(k^{+}+p^{+}\right)^{2}} \chi_{\lambda_{2}}^{\dagger} \chi_{\lambda_{1}}(2 \pi)^{3} \delta_{i j} \delta(k+p+s-q) .
\end{aligned}
$$

Open Access. This article is distributed under the terms of the Creative Commons Attribution License (CC-BY 4.0), which permits any use, distribution and reproduction in any medium, provided the original author(s) and source are credited.

\section{References}

[1] E. Iancu and Y. Mulian, Forward trijet production in proton-nucleus collisions, Nucl. Phys. A 985 (2019) 66 [arXiv:1809.05526] [INSPIRE].

[2] A. Dumitru, A. Hayashigaki and J. Jalilian-Marian, The Color glass condensate and hadron production in the forward region, Nucl. Phys. A 765 (2006) 464 [hep-ph/0506308] [INSPIRE].

[3] J.L. Albacete, A. Dumitru and C. Marquet, The initial state of heavy-ion collisions, Int. J. Mod. Phys. A 28 (2013) 1340010 [arXiv:1302.6433] [INSPIRE].

[4] J.L. Albacete and C. Marquet, Gluon saturation and initial conditions for relativistic heavy ion collisions, Prog. Part. Nucl. Phys. 76 (2014) 1 [arXiv:1401.4866] [InSPIRE].

[5] E. Iancu and R. Venugopalan, The Color glass condensate and high-energy scattering in QCD, Quark-gluon plasma 4 (2003) 249 [INSPIRE].

[6] F. Gelis, E. Iancu, J. Jalilian-Marian and R. Venugopalan, The Color Glass Condensate, Ann. Rev. Nucl. Part. Sci. 60 (2010) 463 [arXiv:1002.0333] [inSPIRE].

[7] I. Balitsky, Operator expansion for high-energy scattering, Nucl. Phys. B 463 (1996) 99 [hep-ph/9509348] [INSPIRE].

[8] Y.V. Kovchegov, Small $x$ F(2) structure function of a nucleus including multiple Pomeron exchanges, Phys. Rev. D 60 (1999) 034008 [hep-ph/9901281] [INSPIRE].

[9] J. Jalilian-Marian, A. Kovner, A. Leonidov and H. Weigert, The BFKL equation from the Wilson renormalization group, Nucl. Phys. B 504 (1997) 415 [hep-ph/9701284] [InSPIRE].

[10] J. Jalilian-Marian, A. Kovner, A. Leonidov and H. Weigert, The Wilson renormalization group for low x physics: Towards the high density regime, Phys. Rev. D 59 (1998) 014014 [hep-ph/9706377] [INSPIRE].

[11] A. Kovner, J.G. Milhano and H. Weigert, Relating different approaches to nonlinear QCD evolution at finite gluon density, Phys. Rev. D 62 (2000) 114005 [hep-ph/0004014] [INSPIRE].

[12] E. Iancu, A. Leonidov and L.D. McLerran, Nonlinear gluon evolution in the color glass condensate. 1., Nucl. Phys. A 692 (2001) 583 [hep-ph/0011241] [InSPIRE].

[13] E. Iancu, A. Leonidov and L.D. McLerran, The Renormalization group equation for the color glass condensate, Phys. Lett. B 510 (2001) 133 [hep-ph/0102009] [INSPIRE].

[14] E. Ferreiro, E. Iancu, A. Leonidov and L. McLerran, Nonlinear gluon evolution in the color glass condensate. 2., Nucl. Phys. A 703 (2002) 489 [hep-ph/0109115] [INSPIRE].

[15] I. Balitsky and G.A. Chirilli, Next-to-leading order evolution of color dipoles, Phys. Rev. D 77 (2008) 014019 [arXiv:0710.4330] [INSPIRE]. 
[16] I. Balitsky and G.A. Chirilli, Rapidity evolution of Wilson lines at the next-to-leading order, Phys. Rev. D 88 (2013) 111501 [arXiv:1309.7644] [INSPIRE].

[17] A. Kovner, M. Lublinsky and Y. Mulian, Jalilian-Marian, Iancu, McLerran, Weigert, Leonidov, Kovner evolution at next to leading order, Phys. Rev. D 89 (2014) 061704 [arXiv: 1310.0378] [INSPIRE].

[18] A. Kovner, M. Lublinsky and Y. Mulian, NLO JIMWLK evolution unabridged, JHEP 08 (2014) 114 [arXiv: 1405.0418] [INSPIRE].

[19] M. Lublinsky and Y. Mulian, High Energy QCD at NLO: from light-cone wave function to JIMWLK evolution, JHEP 05 (2017) 097 [arXiv: 1610.03453] [INSPIRE].

[20] G.A. Chirilli, B.-W. Xiao and F. Yuan, One-loop Factorization for Inclusive Hadron Production in pA Collisions in the Saturation Formalism, Phys. Rev. Lett. 108 (2012) 122301 [arXiv:1112.1061] [INSPIRE].

[21] G.A. Chirilli, B.-W. Xiao and F. Yuan, Inclusive Hadron Productions in pA Collisions, Phys. Rev. D 86 (2012) 054005 [arXiv: 1203.6139] [INSPIRE].

[22] T. Altinoluk, N. Armesto, G. Beuf, A. Kovner and M. Lublinsky, Single-inclusive particle production in proton-nucleus collisions at next-to-leading order in the hybrid formalism, Phys. Rev. D 91 (2015) 094016 [arXiv:1411.2869] [InSPIRE].

[23] I. Balitsky and G.A. Chirilli, Photon impact factor and $k_{T}$-factorization for DIS in the next-to-leading order, Phys. Rev. D 87 (2013) 014013 [arXiv:1207.3844] [INSPIRE].

[24] G. Beuf, Dipole factorization for DIS at NLO: Loop correction to the $\gamma_{T, L}^{*} \rightarrow q \bar{q}$ light-front wave functions, Phys. Rev. D 94 (2016) 054016 [arXiv: 1606.00777] [INSPIRE].

[25] G. Beuf, Dipole factorization for DIS at NLO: Combining the $q \bar{q}$ and $q \bar{q} g$ contributions, Phys. Rev. D 96 (2017) 074033 [arXiv:1708.06557] [INSPIRE].

[26] R. Boussarie, A.V. Grabovsky, L. Szymanowski and S. Wallon, Impact factor for high-energy two and three jets diffractive production, JHEP 09 (2014) 026 [arXiv:1405.7676] [INSPIRE].

$[27]$ R. Boussarie, A.V. Grabovsky, L. Szymanowski and S. Wallon, On the one loop $\gamma^{(*)} \rightarrow q \bar{q}$ impact factor and the exclusive diffractive cross sections for the production of two or three jets, JHEP 11 (2016) 149 [arXiv:1606.00419] [INSPIRE].

[28] R. Boussarie, A.V. Grabovsky, D.Y. Ivanov, L. Szymanowski and S. Wallon, Next-to-Leading Order Computation of Exclusive Diffractive Light Vector Meson Production in a Saturation Framework, Phys. Rev. Lett. 119 (2017) 072002 [arXiv:1612.08026] [INSPIRE].

[29] K. Roy and R. Venugopalan, NLO impact factor for inclusive photon+dijet production in $e+A$ DIS at small x, Phys. Rev. D 101 (2020) 034028 [arXiv:1911.04530] [inSPIRE].

[30] T. Lappi and H. Mäntysaari, Direct numerical solution of the coordinate space Balitsky-Kovchegov equation at next to leading order, Phys. Rev. D 91 (2015) 074016 [arXiv: 1502.02400] [INSPIRE].

[31] A.M. Stasto, B.-W. Xiao and D. Zaslavsky, Towards the Test of Saturation Physics Beyond Leading Logarithm, Phys. Rev. Lett. 112 (2014) 012302 [arXiv:1307.4057] [InSPIRE].

[32] A.M. Staśto, B.-W. Xiao, F. Yuan and D. Zaslavsky, Matching collinear and small $x$ factorization calculations for inclusive hadron production in $p A$ collisions, Phys. Rev. D 90 (2014) 014047 [arXiv:1405.6311] [INSPIRE].

[33] K. Watanabe, B.-W. Xiao, F. Yuan and D. Zaslavsky, Implementing the exact kinematical constraint in the saturation formalism, Phys. Rev. D 92 (2015) 034026 [arXiv:1505.05183] [INSPIRE]. 
[34] B. Ducloué, T. Lappi and Y. Zhu, Single inclusive forward hadron production at next-to-leading order, Phys. Rev. D 93 (2016) 114016 [arXiv:1604.00225] [InSPIRE].

[35] G. Beuf, Improving the kinematics for low- $x$ QCD evolution equations in coordinate space, Phys. Rev. D 89 (2014) 074039 [arXiv:1401.0313] [INSPIRE].

[36] E. Iancu, J.D. Madrigal, A.H. Mueller, G. Soyez and D.N. Triantafyllopoulos, Resumming double logarithms in the QCD evolution of color dipoles, Phys. Lett. B $\mathbf{7 4 4}$ (2015) 293 [arXiv: 1502.05642] [INSPIRE].

[37] E. Iancu, J.D. Madrigal, A.H. Mueller, G. Soyez and D.N. Triantafyllopoulos, Collinearly-improved BK evolution meets the HERA data, Phys. Lett. B 750 (2015) 643 [arXiv: 1507.03651] [INSPIRE].

[38] T. Lappi and H. Mäntysaari, Next-to-leading order Balitsky-Kovchegov equation with resummation, Phys. Rev. D 93 (2016) 094004 [arXiv:1601.06598] [INSPIRE].

[39] Y. Hatta and E. Iancu, Collinearly improved JIMWLK evolution in Langevin form, JHEP 08 (2016) 083 [arXiv: 1606.03269] [INSPIRE].

[40] B. Ducloué, E. Iancu, A.H. Mueller, G. Soyez and D.N. Triantafyllopoulos, Non-linear evolution in QCD at high-energy beyond leading order, JHEP 04 (2019) 081 [arXiv: 1902.06637] [INSPIRE].

[41] B. Ducloué, E. Iancu, G. Soyez and D.N. Triantafyllopoulos, HERA data and collinearly-improved BK dynamics, Phys. Lett. B 803 (2020) 135305 [arXiv:1912.09196] [INSPIRE].

[42] G. Beuf, H. Hänninen, T. Lappi and H. Mäntysaari, Color Glass Condensate at next-to-leading order meets HERA data, Phys. Rev. D 102 (2020) 074028 [arXiv: 2007.01645] [INSPIRE].

[43] E. Iancu, A.H. Mueller and D.N. Triantafyllopoulos, CGC factorization for forward particle production in proton-nucleus collisions at next-to-leading order, JHEP 12 (2016) 041 [arXiv: 1608.05293] [INSPIRE].

[44] S. Catani and F. Hautmann, High-energy factorization and small $x$ deep inelastic scattering beyond leading order, Nucl. Phys. B 427 (1994) 475 [hep-ph/9405388] [inSPIRE].

[45] S. Catani, M. Ciafaloni and F. Hautmann, High-energy factorization and small $x$ heavy flavor production, Nucl. Phys. B 366 (1991) 135 [inSPIRE].

[46] B. Ducloué, T. Lappi and Y. Zhu, Implementation of NLO high energy factorization in single inclusive forward hadron production, Phys. Rev. D 95 (2017) 114007 [arXiv:1703.04962] [INSPIRE].

[47] B. Ducloué D.N. Triantafyllopoulos et al., Use of a running coupling in the NLO calculation of forward hadron production, Phys. Rev. D 97 (2018) 054020 [arXiv:1712.07480] [InSPIRE].

[48] B. Ducloué, H. Hänninen, T. Lappi and Y. Zhu, Deep inelastic scattering in the dipole picture at next-to-leading order, Phys. Rev. D 96 (2017) 094017 [arXiv:1708.07328] [inSPIRE].

[49] C. Marquet, Forward inclusive dijet production and azimuthal correlations in $p(A)$ collisions, Nucl. Phys. A 796 (2007) 41 [arXiv:0708.0231] [INSPIRE].

[50] J.L. Albacete and C. Marquet, Azimuthal correlations of forward di-hadrons in $d+A u$ collisions at RHIC in the Color Glass Condensate, Phys. Rev. Lett. 105 (2010) 162301 [arXiv: 1005.4065] [INSPIRE].

[51] F. Dominguez, C. Marquet, B.-W. Xiao and F. Yuan, Universality of Unintegrated Gluon Distributions at small x, Phys. Rev. D 83 (2011) 105005 [arXiv:1101.0715] [INSPIRE]. 
[52] A. Stasto, B.-W. Xiao and F. Yuan, Back-to-Back Correlations of Di-hadrons in dAu Collisions at RHIC, Phys. Lett. B 716 (2012) 430 [arXiv:1109.1817] [InSPIRE].

[53] E. Iancu and J. Laidet, Gluon splitting in a shockwave, Nucl. Phys. A 916 (2013) 48 [arXiv: 1305.5926] [INSPIRE].

[54] T. Lappi and H. Mantysaari, Forward dihadron correlations in deuteron-gold collisions with the Gaussian approximation of JIMWLK, Nucl. Phys. A 908 (2013) 51 [arXiv:1209.2853] [INSPIRE].

[55] P. Kotko, K. Kutak, C. Marquet, E. Petreska, S. Sapeta and A. van Hameren, Improved TMD factorization for forward dijet production in dilute-dense hadronic collisions, JHEP 09 (2015) 106 [arXiv:1503.03421] [INSPIRE].

[56] C. Marquet, E. Petreska and C. Roiesnel, Transverse-momentum-dependent gluon distributions from JIMWLK evolution, JHEP 10 (2016) 065 [arXiv:1608.02577] [INSPIRE].

[57] A. van Hameren, P. Kotko, K. Kutak, C. Marquet, E. Petreska and S. Sapeta, Forward di-jet production in $p+P b$ collisions in the small-x improved TMD factorization framework, JHEP 12 (2016) 034 [Erratum ibid. 02 (2019) 158] [arXiv: 1607.03121] [INSPIRE].

[58] J.L. Albacete, G. Giacalone, C. Marquet and M. Matas, Forward dihadron back-to-back correlations in pA collisions, Phys. Rev. D 99 (2019) 014002 [arXiv: 1805.05711] [InSPIRE].

[59] A. Metz and J. Zhou, Distribution of linearly polarized gluons inside a large nucleus, Phys. Rev. D 84 (2011) 051503 [arXiv: 1105.1991] [INSPIRE].

[60] A. Dumitru, T. Lappi and V. Skokov, Distribution of Linearly Polarized Gluons and Elliptic Azimuthal Anisotropy in Deep Inelastic Scattering Dijet Production at High Energy, Phys. Rev. Lett. 115 (2015) 252301 [arXiv:1508.04438] [INSPIRE].

[61] T. Altinoluk, N. Armesto, G. Beuf and A.H. Rezaeian, Diffractive Dijet Production in Deep Inelastic Scattering and Photon-Hadron Collisions in the Color Glass Condensate, Phys. Lett. B 758 (2016) 373 [arXiv:1511.07452] [INSPIRE].

[62] Y. Hatta, B.-W. Xiao and F. Yuan, Probing the Small- $x$ Gluon Tomography in Correlated Hard Diffractive Dijet Production in Deep Inelastic Scattering, Phys. Rev. Lett. 116 (2016) 202301 [arXiv: 1601.01585] [INSPIRE].

[63] A. Dumitru, V. Skokov and T. Ullrich, Measuring the Weizsäcker-Williams distribution of linearly polarized gluons at an electron-ion collider through dijet azimuthal asymmetries, Phys. Rev. C 99 (2019) 015204 [arXiv:1809.02615] [INSPIRE].

[64] F. Salazar and B. Schenke, Diffractive dijet production in impact parameter dependent saturation models, Phys. Rev. D 100 (2019) 034007 [arXiv: 1905. 03763] [InSPIRE].

[65] H. Mäntysaari, N. Mueller, F. Salazar and B. Schenke, Multigluon Correlations and Evidence of Saturation from Dijet Measurements at an Electron-Ion Collider, Phys. Rev. Lett. 124 (2020) 112301 [arXiv:1912.05586] [INSPIRE].

[66] A.H. Mueller, B.-W. Xiao and F. Yuan, Sudakov double logarithms resummation in hard processes in the small-x saturation formalism, Phys. Rev. D 88 (2013) 114010 [arXiv: 1308.2993] [INSPIRE].

[67] E. Braidot, Two-particle azimuthal correlations at forward rapidity in STAR, Ph.D. thesis, Utrecht U., 2011. arXiv:1102.0931 [inSPIRE].

[68] PHENIX collaboration, Suppression of back-to-back hadron pairs at forward rapidity in $d+$ Au Collisions at $\sqrt{s_{N N}}=200 \mathrm{GeV}$, Phys. Rev. Lett. 107 (2011) 172301 [arXiv:1105.5112] [INSPIRE]. 
[69] L. Zheng, E.C. Aschenauer, J.H. Lee and B.-W. Xiao, Probing Gluon Saturation through Dihadron Correlations at an Electron-Ion Collider, Phys. Rev. D 89 (2014) 074037 [arXiv: 1403.2413] [INSPIRE].

[70] F. Dominguez, C. Marquet, A.M. Stasto and B.-W. Xiao, Universality of multiparticle production in QCD at high energies, Phys. Rev. D 87 (2013) 034007 [arXiv:1210.1141] [INSPIRE].

[71] F. Dominguez, A.H. Mueller, S. Munier and B.-W. Xiao, On the small-x evolution of the color quadrupole and the Weizsäcker-Williams gluon distribution, Phys. Lett. B 705 (2011) 106 [arXiv: 1108.1752] [INSPIRE].

[72] E. Iancu and D.N. Triantafyllopoulos, Higher-point correlations from the JIMWLK evolution, JHEP 11 (2011) 105 [arXiv:1109.0302] [INSPIRE].

[73] E. Iancu and D.N. Triantafyllopoulos, JIMWLK evolution in the Gaussian approximation, JHEP 04 (2012) 025 [arXiv: 1112.1104] [INSPIRE].

[74] J.P. Blaizot, F. Gelis and R. Venugopalan, High-energy $p A$ collisions in the color glass condensate approach. 2. Quark production, Nucl. Phys. A 743 (2004) 57 [hep-ph/0402257] [INSPIRE].

[75] A. Dumitru, J. Jalilian-Marian, T. Lappi, B. Schenke and R. Venugopalan, Renormalization group evolution of multi-gluon correlators in high energy QCD, Phys. Lett. B 706 (2011) 219 [arXiv: 1108.4764] [INSPIRE].

[76] T. Altinoluk, N. Armesto, G. Beuf and A. Moscoso, Next-to-next-to-eikonal corrections in the CGC, JHEP 01 (2016) 114 [arXiv:1505.01400] [INSPIRE].

[77] T. Altinoluk and A. Dumitru, Particle production in high-energy collisions beyond the shockwave limit, Phys. Rev. D 94 (2016) 074032 [arXiv: 1512.00279] [InSPIRE].

[78] T. Altinoluk, G. Beuf, A. Czajka and A. Tymowska, Quarks at next-to-eikonal accuracy in the CGC I: Forward quark-nucleus scattering, arXiv:2012.03886 [INSPIRE].

[79] G.A. Chirilli, Sub-eikonal corrections to scattering amplitudes at high energy, JHEP 01 (2019) 118 [arXiv:1807.11435] [INSPIRE].

[80] P. Agostini, T. Altinoluk and N. Armesto, Non-eikonal corrections to multi-particle production in the Color Glass Condensate, Eur. Phys. J. C 79 (2019) 600 [arXiv: 1902.04483] [INSPIRE].

[81] A. Kovner, M. Lublinsky and H. Weigert, Treading on the cut: Semi inclusive observables at high energy, Phys. Rev. D 74 (2006) 114023 [hep-ph/0608258] [INSPIRE].

[82] A. Kovner and M. Lublinsky, One gluon, two gluon: Multigluon production via high energy evolution, JHEP 11 (2006) 083 [hep-ph/0609227] [INSPIRE].

[83] E. Iancu and D.N. Triantafyllopoulos, JIMWLK evolution for multi-particle production in Langevin form, JHEP 11 (2013) 067 [arXiv:1307.1559] [INSPIRE]. 